\title{
EXCHANGE DESIGN AND EFFICIENCY
}

\author{
MARZENA ROSTEK \\ Department of Economics, University of Wisconsin-Madison \\ Ji HEE YOON \\ Department of Economics, University College London
}

\begin{abstract}
Most assets clear independently rather than jointly. This paper presents a model based on the uniform-price double auction which accommodates arbitrary restrictions on market clearing, including independent clearing across assets (allowed when demand for each asset is contingent only on the price of that asset) and joint market clearing for all assets (required when demand for each asset is contingent on the prices of all assets). Additional trading protocols for traded assets-neutral when the market clears jointly - are generally not redundant innovations, even if all traders participate in all protocols. Multiple trading protocols that clear independently can be designed to be at least as efficient as joint market clearing for all assets. The change in price impact brought by independence in market clearing can overcome the loss of information, and enhance diversification and risk sharing. Except when the market is competitive, market characteristics should guide innovation in trading technology.
\end{abstract}

KEYWORDS: Imperfect competition, trading technology, market design, innovation, decentralized market, liquidity, price impact, uniform-price auction, efficiency.

\section{INTRODUCTION}

TODAY'S FINANCIAL MARKETS are composed of coexistent trading protocols for the same or distinct assets. Venues for financial securities clear independently, and typically the assets traded in each venue do as well: an order submitted for one asset cannot be made contingent on the prices of other assets. In some markets, such as those for spectrum, electricity, and electronic trading platforms for financial assets, traders can express their demand for one asset contingent on the prices of other assets. ${ }^{1}$ When available, however,

Marzena Rostek:mrostek@ssc.wisc.edu

Ji Hee Yoon: jihee.yoon@ucl.ac.uk

We would like to thank the anonymous referees and numerous colleagues and participants at the Conference on "What We Know About Information in Financial Markets" (UC Santa Barbara, LEAF), the Cowles Conference on Economic Theory, the Philadelphia Search and Matching Conference (Federal Reserve Bank of Philadelphia), Conference on Finance-Related Theory and Experiments (NYU), Conference on "The Changing Regulatory and Technological Landscape of Market Structure" (Vanderbilt, The Financial Markets Research Center), the Conference on Advances in Mechanism and Market Design (NYU Abu Dhabi), the 5th European Meeting on Networks (UCL), the Spring Workshop in Economic Theory (Queen Mary University of London), and seminar participants at Arizona State University, University of Calgary (Finance), Caltech, Carnegie Mellon University/Pittsburgh University, University of Chicago, Columbia University, ECARES, The Federal Reserve Board, HEC Paris (Finance), Higher School of Economics \& ICEF (Moscow), IAQF \& Thalesians (New York), INSEAD (Finance), Ohio State University, New Economic School (Moscow), Paris School of Economics, Princeton University, Stanford GSB, Stockholm School of Economics (Economics, Finance), UCL, UCSD, University of Cambridge, and Washington University (St. Louis) for extremely insightful comments and suggestions. Rostek gratefully acknowledges the financial support from NSF Grant SES-1851857. Yoon acknowledges the financial support of the University of Wisconsin-Madison and UCL.

${ }^{1}$ For example, Active Trader Pro, Etrade, Street Smart, Tradehawk. Variants of cross-asset conditioning are available in futures and options markets (e.g., multi-leg orders). The Regulation National Market System and Unlisted Trading Privileges in U.S. stock exchanges de facto induce contingent demand schedules; however, 
such contingent orders allow cross-asset conditioning among a limited number of assets. Feasibility might provide one rationale as to why cross-asset conditioning is relatively uncommon in practice-with contingent schedules, the market-clearing prices must be determined jointly for all assets, thus requiring coordination in market clearing among market makers or trading venues that are private entities. Advances in technology have increased interest in cross-asset conditioning. Indeed, electronic trading platforms such as those listed in ft. 1 innovate on such orders.

The objective of this paper is twofold. First, we investigate the implications of independent market clearing for the equilibrium and for welfare. Second, we examine the innovations in trading technology_-defined by changes in market clearing-that cross-asset conditioning makes possible. As we will show, regulation that promotes joint clearing for some assets, if applied in disregard of market characteristics, can lower welfare in the Pareto sense. Moreover, multiple exchanges that clear independently can be designed to be at least as efficient as joint clearing of all assets irrespective of the characteristics of assets and traders. Thus, joint market clearing of all assets is not essential and can be suboptimal.

We dispense with the assumption that demand schedules are contingent-on which the standard competitive and imperfectly competitive models of equilibrium and asset pricing are based-in the canonical uniform-price double auction for $I<\infty$ strategic traders and $K<\infty$ assets (e.g., Wilson (1979), Klemperer and Meyer (1989), Kyle (1989), Vives (2011)). Our analysis is cast in the quadratic-Gaussian setting. Traders have private information about their endowments, which are independent across assets and possibly correlated across traders. The model encompasses contingent schedules $q_{k}^{i, c}(\cdot): \mathbb{R}^{K} \rightarrow \mathbb{R}$, which are standard in theory but less so in practice, specifying the quantities demanded of each asset for any realization of the price vector (i.e., joint market clearing for all assets).

We first examine markets with uncontingent schedules $q_{k}^{i}(\cdot): \mathbb{R} \rightarrow \mathbb{R}$, each specifying the quantities demanded for any price realization of a given asset (i.e., assets clear independently). To accommodate innovation in trading technology and more general market structures, we then generalize the model in two ways. Specifically, we permit arbitrary restrictions on cross-asset demand conditioning "between" uncontingent and contingent and allow an asset to be traded in multiple venues. A market structure consists of exchanges, each defined by the subset of the $K$ assets traded there; all traders participate in all exchanges (see also ft. 12). Demand schedules condition on the prices of the assets traded in an exchange and not on those in other exchanges, and the market clears independently across exchanges. It is convenient to identify independent market clearing (i.e., a uniform-price trading protocol) with an exchange in the model. An exchange can represent either a trading protocol within a trading platform or the platform itself.

The methodological contribution of this paper is the characterization of the Bayesian Nash equilibrium in market structures with limited cross-asset conditioning. When a trader's demand schedules are not contingent on the prices of all assets, they depend on the expected (rather than realized) trades of the assets in other exchanges. Due to cross-asset inference, the coefficients of a trader's own best-response demand schedules must be characterized as a fixed point across assets. ${ }^{2}$ Additionally, the price impact is no

analogous rules do not apply in markets for other asset classes or stock markets abroad (see Budish, Lee, and Shim (2020)).

${ }^{2}$ In a multivariate optimization problem, contingent or uncontingent, a trader's first-order conditions define a fixed point for the trader's best-response demand schedules across assets. With demand contingent on the common price vector, the first-order conditions can be written as a single matrix equation and solved for the 
longer a sufficient statistic for a trader's residual supply-the joint distribution of other traders' initial holdings of all assets matters as well.

We show that the equilibrium fixed point in demand schedules is equivalent to a fixed point in price impact matrices alone. That is, we endogenize all demand coefficientsincluding expected trades and the distributions of residual supplies-as functions of price impacts (Theorem 1). We prove the existence of a symmetric linear Bayesian Nash equilibrium in the uniform-price double auction (Theorem 2) and equilibrium uniqueness for symmetric markets (Proposition S1 in Appendix C of the Supplemental Material (Rostek and Yoon (2021))). ${ }^{3}$

The paper's second contribution is its implications of independence in market clearing for equilibrium, welfare, and design, which underscore the role of imperfect competition. Why would trading platforms not allow traders to condition their demands on more information from other venues or trading protocols within the platform? In competitive markets $(I \rightarrow \infty)$, joint market clearing would be weakly more efficient than any other market structure regardless of the characteristics of assets and traders, as contingent schedules prevent inference error across exchanges. We show that in imperfectly competitive markets $(I<\infty)$, welfare can be improved by moving away from the contingent design, even if that means that when placing an order for an asset one cannot use all information from all other assets. The key is that independent market clearing can lower the trading costs associated with per-unit price impact for a given asset and/or across assets. Respectively, multi-venue trading changes the traders' ability to share an asset's risk and diversify risk across assets. Price impact is of first-order importance for traders' incentives in financial markets (see, e.g., the survey by Rostek and Yoon (2020b)).

Central to the effects of multi-venue trading that have no analogues with joint clearing is that multi-venue trading severs the proportionality between the equilibrium price impact - and hence cross-asset demand substitution-and the fundamental asset covariance that holds with contingent trading. First, the cross-venue price impact becomes zero, ${ }^{4}$ which may or may not be conducive to efficiency (Example 1). Second, with multiple assets per venue, cross-asset price impacts are asymmetric except when asset covariance and market structure are both symmetric across assets. Underlying the asymmetry are the cross-asset inference effects brought by independence in market clearing. We provide the comparative statics of price impact, which can be higher or lower than in the contingent market, with respect to the asset correlation and number of traders (Theorem 3, Proposition S2 in Appendix C.1).

We present three main results. First, once one departs from the assumption that demands are contingent, innovation in trading technology ${ }^{5}$ which would be neutral for traders' equilibrium payoffs with joint clearing (or have no counterparts) is no longer redundant. We provide a complete characterization of non-neutral innovation. Proposition 4 identifies nonredundant innovation with a change in per-unit price impact. Intu-

quantity vector pointwise with respect to the price vector. Thus, the best-response demand coefficients need not be characterized as a fixed point.

${ }^{3}$ With respect to asset correlations, trading needs, and market structure.

${ }^{4}$ Nevertheless, equilibrium behavior and outcome (i.e., prices and trades) are not independent across trading venues - unless the asset payoffs are independent.

${ }^{5}$ Or in market structure, depending on whether market clearing is interpreted as applying to trading venues or the trading protocols that they provide-the model accommodates both. Neither alters the traders' endowments, assets' net supply (which we assume to be zero, for simplicity), or participation. Thus, invoking the notion of spanning: in contrast to the contingent model, innovation in market clearing for the traded assets which does not change their payoff span is no longer redundant with independent market clearing. 
itively, an innovation is not redundant if it alters the trading costs (or inference) across assets. Not all new trading protocols affect welfare (Theorem 4).

Moreover, there is more nonredundant innovation in imperfectly competitive markets. Namely, independent market clearing motivates two types of innovations that are nonredundant: innovations that reduce inference error for all assets (e.g., the linking of existing trading protocols by merging their assets or the listing of an asset in a trading protocol where it was not previously traded) and innovations that alter inference across assets without letting the demand for any asset be contingent on prices of additional assets (e.g., opening another venue for a traded asset, Example 3). If the market were competitive, the latter type of innovation would be redundant irrespective of demand conditioning.

Second, markets with multiple trading protocols that clear independently can be at least as efficient as a single exchange that clears all assets jointly. We show that one can design a market with multiple protocols - none of which clears all assets - that can function like a single exchange. That is, equilibrium is ex post even if demand is not contingent on the prices of all assets in any exchange. Thus, innovation in trading technology can bound welfare at the corresponding contingent level—with no knowledge of traders' preferences and endowments or asset distribution. Such designs involve sufficiently many trading protocols for different assets, which enable the conditioning variables to eliminate inference errors in traders' expectations.

This equivalence result also characterizes the scope for nonredundant innovation that can be introduced in a market. For a market structure to implement an equilibrium with joint clearing, one venue per pair of assets suffices - the maximal number of nonredundant protocols is $\frac{K(K-1)}{2}$.

Third, we ask which designs can strictly improve welfare relative to the welfare bound implemented by the designs equivalent to joint clearing. Hinting at the diversity of the trading protocols in practice, the market structure in which all assets clear jointly (i.e., contingent demands or a payoff-equivalent design) is not generally efficient; nor is the market structure in which every asset is traded in a separate venue (i.e., uncontingent demands) efficient regardless of the market characteristics. In symmetric trading environments, the extreme uncontingent/contingent market structures are optimal with asset payoff substitutabilities/complementarities, respectively. Sufficient heterogeneity in either assets' covariances or traders' trading needs across assets can favor a market structure which is "intermediate" between contingent or uncontingent. In this case, inducing asymmetries in trading costs can be beneficial.

Our results recognize that the welfare-enhancing exchange design should respond to the number of traders, and joint substitution of the asset payoffs and trading needs of market participants across assets. ${ }^{6}$ Even if assets' payoffs are all either symmetric substitutes or complements, efficient design depends on whether the market is "one-sided" - that is, traders want to buy or sell all assets (e.g., the primary market for Treasury securities) —or some assets are demanded while others are supplied (e.g., inter-dealer markets). In imperfectly competitive markets, given the assets and traders, a market structure with multiple venues is more efficient than joint clearing for some distributions of endowments (Proposition 3). In fact, any demerger (i.e., breaking up a single exchange for all assets into multiple venues) can increase welfare.

One might wonder whether the independence in market clearing across trading venues has any effects, given that, when trading is dynamic, traders can often condition their

\footnotetext{
${ }^{6}$ In a market with multiple venues, all innovations are neutral if and only if the payoffs of all assets are either perfectly correlated (i.e., cross-asset inference is perfect) or independent (i.e., cross-asset inference is absent), or (by design) equilibrium is ex post.
} 
demands in one trading venue on past outcomes from other venues. Conditioning on past outcomes allows information from past shocks to be (at least partially) incorporated into traders' demands-contingent or not. Our paper investigates how independence in market clearing affects the way current-round shocks impact behavior and outcomes. ${ }^{7}$ Our results thus indicate a role for opaqueness in the form of independent market clearing (embodied in restrictions on cross-asset conditioning), which has implications distinct from transparency requirements (i.e., conditioning on past outcomes).

Other related literature. Our paper contributes to the literature on imperfectly competitive trading. ${ }^{8}$ To our knowledge, we are the first to examine equilibrium and welfare outcomes with arbitrary restrictions on cross-asset conditioning and to characterize the (non)redundant exchange design. In fact, little is known about markets with multiple heterogeneous assets outside of settings with ex post equilibria. One precedent is Cespa (2004), who examined price informativeness in competitive uncontingent markets with two assets and noise traders. Contemporaneously, in a model with two assets and random supply, Wittwer (2021) showed that traders trade the same amounts with contingent and uncontingent demands if and only if traders' private signals are perfectly correlated and supplies are either zero or perfectly correlated across assets. In a one-asset model with strategic traders and noise traders, Chen and Duffie (2021) showed that additional venues increase welfare relative to one venue and the welfare-maximizing number of exchanges is finite.

Apart from financial market applications, the techniques we introduce will be useful to researchers studying games in which agents interact through contracts over multiple goods, actions, or characteristics. One application is to package auctions with large traders, who have price impact. The literature on package bids has supported package designs when there are complementarities in traders' demands. Our results show, however, that design recommendations should be qualified in markets with price impact and multi-unit demands, even when traders and assets are symmetric. Moreover, package bids can be implemented without the requirement that schedules must be contingent.

The problem in which players submit uncontingent demand schedules in different trading venues is also related to those studied by the literature on "island" models (in competitive markets) and, more generally, the approach based on Nash-in-Nash. ${ }^{9}$ A typical

\footnotetext{
${ }^{7}$ With the gains from trade renewed by shocks (to endowment or information), if demand schedules are contingent and traders are price-takers $(I \rightarrow \infty)$, the outcome will be efficient in every round. In a dynamic model with imperfectly competitive traders $(I<\infty)$, multiple rounds are needed to realize the gains from trade from a given round's shock. The effects of limited conditioning that we identify will be present in all rounds. From the literature on dynamic trading (which is based on contingent demands or one-asset markets; e.g., Du and Zhu (2017b), Rostek and Yoon (2019), see also the survey by Rostek and Yoon (2020b)), two results can be extrapolated beyond contingent demands. First, whether the inefficiency of trade that stems from limited demand conditioning can be eliminated in some limit depends on the relative frequencies of the shocks renewing the gains from trade, market-clearing, and payoff realization (consumption). With shocks to traders' valuations occurring as frequently as trading rounds, the inefficiencies will generally be present even with continuous trading. Second, apart from its contemporaneous effects (the focus of this paper), limited conditioning will change temporal effects on price impact (see Lyu, Rostek, and Yoon (2021)).

${ }^{8}$ For example, Kyle (1989), Vayanos (1999), Vives (2011), Garleanu and Pedersen (2013), Rostek and Weretka (2015), Sannikov and Skrzypacz (2016), Du and Zhu (2017a, 2017b), Kyle, Obizhaeva, and Wang (2017), Duffie (2018), Kyle and Lee (2018), Zhu (2018a, 2018b), Antill and Duffie (2021), Bergemann, Heumann, and Morris (2021), Zhang (2021).

${ }^{9}$ This solution concept, introduced by Horn and Wolinsky (1988), has become popular in the structural analysis of decentralized markets. As in this paper's model, the applications of Nash-in-Nash have typically considered negotiated contracts, given the set of agreements. See, for example, Collard-Wexler, Gowrisankaran, and
} 
context where Nash-in-Nash has been applied is surplus division in bargaining or contracting with externalities - across contracts and agents — when negotiations are simultaneous. Likewise, in this paper, the demand schedules that a player submits simultaneously for different assets are essentially contracts specifying the quantities demanded as a function of a subset of prices. There are two differences. In Nash-in-Nash, a player agrees to the price in one contract while holding fixed (i) the prices in his other contracts ${ }^{10}$ and (ii) the prices to which other players agree. By its virtue of treating prices as contingent variables in traders' demands, the (noncooperative) game in demand functions allows accounting for the cross-contract and cross-player externalities in a Bayesian Nash equilibriumwithout employing the Nash-in-Nash counterfactual (which holds prices fixed in other contracts) or restricting how beliefs can change off equilibrium (e.g., passive beliefs; Hart and Tirole (1990)). ${ }^{11}$ Our model complements the Nash-in-Nash approach in applications where inefficiencies in surplus sharing arise due to limited inference and imperfect competition, and contracts involve multiple assets with cross-asset externalities. Accounting for imperfect competition along with private information sheds light on how the design of contracts over which agents bargain can enhance the equilibrium surplus when there are cross-contract externalities. With price-taking behavior, contingent contracts are efficient.

Our paper also contributes to the literature on decentralized trading. Markets with contingent schedules are centralized because a single market clearing applies to all assets. Accordingly, a market in which assets are traded in separate venues that clear independently is decentralized. The assumption that schedules are contingent is the only assumption of the centralized market model that we relax. In particular, assuming that all traders trade all assets with all other traders allows us to focus on those effects of decentralized trading that are due to incomplete conditioning as opposed to incomplete participation. ${ }^{12}$ The literature recognizes several arguments as to why decentralized trading might be more efficient: it may improve traders' learning about the asset value (Babus and Kondor (2018)) or asset price (Zhu (2014)); it may reduce inefficient screening (Glode and Opp (2016)) or inefficient information aggregation (Kawakami (2017)); it may redistribute risk towards less risk-averse traders (Malamud and Rostek (2017)); and it may be more stable than the centralized market (Peivandi and Vohra (2021)). This paper contributes another argument: even if risk preferences are the same among all traders, decentralized trading

Lee (2019) and references there. We are grateful to an anonymous referee for suggesting we explore the link to the literature on "island" models.

${ }^{10}$ This is typically justified using the "delegated agent" interpretation: a player involved in multiple bilateral bargains relies on separate agents for each negotiation, and these agents cannot communicate with one another while bargaining.

${ }^{11}$ With price-elastic demands, all price realizations occur in equilibrium for some realizations of endowments.

${ }^{12}$ In the centralized market assumption, two assumptions are implicit. First, demand conditioning is complete (i.e., demands are contingent); then, a single aggregation applies to all assets. Second, trader participation in the market is complete in the sense that each trader trades all assets with all other traders. A growing literature on decentralized trading has explored the implications of limited participation modeled as fixed or random (hyper)graphs (e.g., Gale (1986a, 1986b), Kranton and Minehart (2001), Duffie, Garleanu, and Pedersen (2005), Vayanos and Weill (2008), Afonso and Lagos (2015), Gofman (2018), Atkeson, Eisfeldt, and Weill (2015), Elliott (2015), Choi, Galeotti, and Goyal (2017), Condorelli, Galeotti, and Renou (2017), Hugonnier, Lester, and Weill (2020), Malamud and Rostek (2017), and Chang and Zhang (2019)). Babus and Kondor (2018), Babus and Parlatore (2021), and Malamud and Rostek (2017) studied markets with limited participation and contingent contracts. Interestingly, with decentralized trading in the sense of limited demand conditioning (e.g., this paper) as well as limited participation, the equilibrium price covariance and price impact are not proportional to the asset covariance (see Malamud and Rostek (2017)). Yet, the effects on price impact, as well as the underlying mechanisms, are distinct. Indeed, with limited participation and contingent schedules, equilibrium is ex post. 
may improve risk sharing and/or diversification by lowering the trading costs that are due to price impact. ${ }^{13}$

\section{MODEL}

Notation. We use the following notation: $\left(x_{k}\right)_{k}$ is a vector in which the $k$ th element is $x_{k}$, and $\left(y_{k \ell}\right)_{k, \ell}$ is a matrix such that the $(k, \ell)$ th element is $y_{k \ell}$; sets of the respective elements are denoted by $\left\{x_{k}\right\}_{k}$ and $\left\{y_{k \ell}\right\}_{k, \ell}$. Also, $\operatorname{diag}\left(x_{k}\right)_{k}=\operatorname{diag}\left(x_{1}, \ldots, x_{K}\right)$ is a diagonal matrix in $\mathbb{R}^{K \times K}$ where the $k$ th diagonal element is $x_{k}$. The $(k, \ell)$ th element of matrix $\mathbf{M}$ is denoted by $m_{k \ell}$, and the $k$ th row is denoted by $\mathbf{M}_{k}$. To distinguish them from scalar variables, vectors and matrices are denoted in bold, and matrices are capitalized.

Market: traders, assets, and exchanges. Consider a market with $I \geq 3$ traders who trade $K$ risky assets in $N$ exchanges. An exchange is defined by the assets traded (listed) there; all traders participate in all exchanges. In Section 3, to ease exposition, we focus on markets with one asset per exchange, $N=K$; in Sections 4 and 5, we consider exchanges with multiple assets (Definition 4). We index traders by $i$, assets by $k$, and exchanges by $n$.

The payoffs of the $K$ risky assets are jointly normally distributed $\mathbf{r}=\left(r_{k}\right)_{k} \sim \mathcal{N}(\boldsymbol{\delta}, \mathbf{\Sigma})$ with a vector of expected payoffs $\boldsymbol{\delta}=\left(\delta_{k}\right)_{k} \in \mathbb{R}^{K}$ and a positive semi-definite covariance matrix $\Sigma \in \mathbb{R}^{K \times K}$. There is also a riskless asset with a zero interest rate (a numéraire).

Each trader $i$ has quadratic utility in the quantity of risky assets (mean-variance):

$$
u^{i}\left(\mathbf{q}^{i}\right)=\boldsymbol{\delta} \cdot\left(\mathbf{q}^{i}+\mathbf{q}_{0}^{i}\right)-\frac{\alpha^{i}}{2}\left(\mathbf{q}^{i}+\mathbf{q}_{0}^{i}\right) \cdot \boldsymbol{\Sigma}\left(\mathbf{q}^{i}+\mathbf{q}_{0}^{i}\right),
$$

where $\mathbf{q}^{i}=\left(q_{k}^{i}\right)_{k} \in \mathbb{R}^{K}$ is trade, $\mathbf{q}_{0}^{i}=\left(q_{0, k}^{i}\right)_{k} \in \mathbb{R}^{K}$ represents the units of risky assets with which trader $i$ is initially endowed, and $\alpha^{i} \in \mathbb{R}_{+}$is trader $i$ 's risk aversion. Endowments $\left\{\mathbf{q}_{0}^{i}\right\}_{i}$ are traders' private information and are independent of asset payoffs $\mathbf{r}$. Gains from trade come from risk sharing and diversification: endowments are heterogeneous. All traders are strategic.

In keeping with the literature, to ensure that the per-capita aggregate endowment (equivalently, price) is random in the limit large market $(I \rightarrow \infty)$, we allow for the common value component $\mathbf{q}_{0}^{c v}=\left(q_{0, k}^{c v}\right)_{k} \in \mathbb{R}^{K}$ in traders' endowments. For each asset $k$, privately known endowments $\left\{q_{0, k}^{i}\right\}_{i}$ are correlated among traders through $q_{0, k}^{c v} \sim$ $\mathcal{N}\left(E\left[q_{0, k}^{c v}\right], \sigma_{c v}\right)$ : for each $i$

$$
q_{0, k}^{i}=q_{0, k}^{c v}+q_{0, k}^{i, p v}, \quad q_{0, k}^{i, p v} \sim \mathcal{N}\left(E\left[q_{0, k}^{i, p v}\right], \sigma_{p v}\right)
$$

where $q_{0, k}^{i, p v}$ are independent across $i$ and $k .{ }^{14}$ Trader $i$ knows his endowment $\mathbf{q}_{0}^{i}$ but not its components $\mathbf{q}_{0}^{c v}$ or $\mathbf{q}_{0}^{i, p v}=\left(q_{0, k}^{i, p v}\right)_{k} \in \mathbb{R}^{K}$. The endowments $\left\{q_{0, k}^{i}\right\}_{i}$ and the common value

\footnotetext{
${ }^{13}$ Lester, Shourideh, Venkateswaran, and Zetlin-Jones $(2018,2019)$ examined the joint effects of an information friction and market power (induced by a search friction) in over-the-counter markets.

${ }^{14}$ The common value component in $\left\{\mathbf{q}_{0}^{i}\right\}_{i}$ affects the magnitude of inference coefficients but does not affect any results qualitatively. Assuming (correlated) private values better isolates the effects that are key to nonreundancy of innovation, which do not rely on inference related to common values. The approach to characterize the linear equilibrium would go through with common values.
} 
$q_{0, k}^{c v}$ are independent across assets $k \cdot{ }^{15}$ We let $\overline{\mathbf{q}}_{0} \equiv \frac{1}{I} \sum_{j} \mathbf{q}_{0}^{j} \in \mathbb{R}^{K}$ denote the aggregate endowment and define $\sigma_{0} \equiv \frac{\sigma_{c v}+\frac{1}{T} \sigma_{p v}}{\sigma_{c v}+\sigma_{p v}} \in \mathbb{R}$.

Double auction. Each exchange is organized as a uniform-price double auction in which traders submit strictly downward-sloping ${ }^{16}$ (net) demand schedules. For $q_{k}^{i}>0$, trader $i$ is a buyer of asset $k$; for $q_{k}^{i}<0$, he is a seller. We first consider two types of schedules: contingent and uncontingent. In Section 4, we analyze arbitrary cross-asset conditioning.

DEFINITION 1-Contingent and Uncontingent Schedules: In a double auction with contingent schedules, each trader $i$ submits $K$ demand functions $\mathbf{q}^{i, c}(\cdot) \equiv\left(q_{1}^{i, c}(\mathbf{p}), \ldots\right.$, $\left.q_{K}^{i, c}(\mathbf{p})\right)$, each $q_{k}^{i, c}(\cdot): \mathbb{R}^{K} \rightarrow \mathbb{R}$ specifying the quantity of asset $k$ demanded for any price vector $\mathbf{p}=\left(p_{1}, \ldots, p_{K}\right)$.

In a double auction with uncontingent schedules, each trader $i$ submits $K$ demand functions $\mathbf{q}^{i}(\cdot) \equiv\left(q_{1}^{i}\left(p_{1}\right), \ldots, q_{K}^{i}\left(p_{K}\right)\right)$, each $q_{k}^{i}(\cdot): \mathbb{R} \rightarrow \mathbb{R}$ specifying the quantity of asset $k$ demanded for any price $p_{k}$.

How the market clears is determined by demand conditioning. With uncontingent schedules, the market clears independently across assets: the market-clearing price $p_{k}$ sets the aggregate net demand in each exchange $k$ to zero, $\sum_{i} q_{k}^{i}\left(p_{k}\right)=0$. With contingent schedules, the $K$ assets clear jointly: the equilibrium price vector is determined by $\sum_{i} \mathbf{q}^{i, c}\left(p_{1}, \ldots, p_{K}\right)=\mathbf{0} \in \mathbb{R}^{K}$. With either type of schedule, trader $i$ trades $\left\{q_{k}^{i}\right\}_{k}$, pays $\sum_{k} p_{k} q_{k}^{i}$, and receives a payoff of $u^{i}\left(\mathbf{q}^{i}\right)-\mathbf{p} \cdot \mathbf{q}^{i}$.

Equilibrium. We study the Bayesian Nash equilibrium in linear demand schedules (hereafter, equilibrium).

Definition 2-Equilibrium: A profile of (net) demand schedules $\left\{\left\{q_{k}^{i}(\cdot)\right\}_{k}\right\}_{i}$ is a Bayesian Nash equilibrium if, for each $i,\left\{q_{k}^{i}(\cdot)\right\}_{k}$ maximizes the expected payoff:

$$
\max _{\left\{q_{k}^{i}(\cdot)\right\}_{k}} E\left[\boldsymbol{\delta} \cdot\left(\mathbf{q}^{i}+\mathbf{q}_{0}^{i}\right)-\frac{\alpha^{i}}{2}\left(\mathbf{q}^{i}+\mathbf{q}_{0}^{i}\right) \cdot \mathbf{\Sigma}\left(\mathbf{q}^{i}+\mathbf{q}_{0}^{i}\right)-\mathbf{p} \cdot \mathbf{q}^{i} \mid \mathbf{q}_{0}^{i}\right],
$$

given the schedules of other traders $\left\{\left\{q_{k}^{j}(\cdot)\right\}_{k}\right\}_{j \neq i}$ and market clearing $\sum_{j} q_{k}^{j}(\cdot)=0$ for all $k$.

As is well known, in markets with contingent schedules, equilibrium is invariant to the distribution of private endowments; that is, the linear Bayesian Nash equilibrium with (possibly correlated) private endowments has an ex post property ${ }^{17}$ The contingent schedule allows a trader to choose his demand for each asset as a function of all prices

\footnotetext{
${ }^{15}$ For simplicity, we assume the symmetry of endowments' variance across traders and their independence across assets; the results hold qualitatively without these assumptions. Our equilibrium characterization in Appendix A allows for correlated endowments across assets that are symmetrically correlated across traders.

${ }^{16}$ That is, the Jacobian of demand schedules $\frac{\partial \mathbf{q}^{i}(\cdot)}{\partial \mathbf{p}}=\left(\frac{\partial q_{k}^{i}(\cdot)}{\partial p_{\ell}}\right)_{k, \ell} \in \mathbb{R}^{K \times K}$ is negative semi-definite. This rules out trivial equilibria with no trade.

${ }^{17}$ Equilibrium is linear if schedules have the functional form of $\mathbf{q}^{i}(\cdot)=\boldsymbol{a}^{i}+\mathbf{B}^{i} \mathbf{q}_{0}^{i}+\mathbf{C}^{i} \mathbf{p}$. Equilibrium is ex post if equilibrium schedules $\left\{q_{k}^{i}\left(\cdot ; \mathbf{q}_{0}^{i}\right)\right\}_{k}$ are optimal for all $i$, given endowment realizations for all traders $\left\{\mathbf{q}_{0}^{j}\right\}_{j}$ :

$$
\left\{q_{k}^{i}\left(\cdot ; \mathbf{q}_{0}^{i}\right)\right\}_{k}=\arg \max _{\left\{q_{k}^{i}(\cdot)\right\}_{k}} E\left[\boldsymbol{\delta} \cdot\left(\mathbf{q}^{i}+\mathbf{q}_{0}^{i}\right)-\frac{\alpha^{i}}{2}\left(\mathbf{q}^{i}+\mathbf{q}_{0}^{i}\right) \cdot \mathbf{\Sigma}\left(\mathbf{q}^{i}+\mathbf{q}_{0}^{i}\right)-\mathbf{p} \cdot \mathbf{q}^{i} \mid\left\{\mathbf{q}_{0}^{j}\right\}_{j}\right] .
$$
}


to be realized, which map one-to-one to realizations of quantities traded of other assets. With uncontingent schedules, equilibrium is not generally ex post. Given the quasilinearquadratic utility, traders face uncertainty both with respect to the price and payoff; their expected payoff (2) penalizes the latter, but not the former.

Competitive market. The competitive market will often serve as a benchmark when evaluating the effects of incomplete conditioning with imperfectly competitive traders. ${ }^{18}$

DEFINITION 3-Competitive Market, Competitive Equilibrium: Consider a market with $I<\infty$ traders. The competitive market is the limit game as $I \rightarrow \infty$, holding fixed all other primitives. Letting $\left\{\mathbf{q}^{i, I}(\cdot)\right\}_{i}$ be the equilibrium in the market with $I<\infty$ traders, the competitive equilibrium $\left\{\mathbf{q}^{i}(\cdot)\right\}_{i}$ is the limit of equilibria $\left\{\mathbf{q}^{i, I}(\cdot)\right\}_{i}$ as $I \rightarrow \infty$ :

$$
\mathbf{q}^{i}(\cdot)=\lim _{I \rightarrow \infty} \mathbf{q}^{i, I}(\cdot) \quad \forall i .
$$

\section{EQUILIBRIUM: CONTINGENT VERSUS UNCONTINGENT DEMANDS}

In this section, we characterize equilibrium in markets with uncontingent demands (Proposition 2, Theorem 1, and Corollary 1). For the sake of comparison, we also review equilibrium with contingent demands.

Although the contingent and uncontingent models are quite different, equilibria in both models can be characterized through parallel conditions (Propositions 1 and 2). First, a key argument (Lemma S2 in Appendix B of the Supplemental Material) shows that the well-known equivalence between individual trader optimization in demand functions (2) and pointwise optimization with respect to the realizations of $\mathbf{p} \in \mathbb{R}^{K}$ in the contingent model also holds in the uncontingent model with respect to the realizations of the relevant contingent variable, $p_{k} \in \mathbb{R}$. Both pointwise problems are motivated by the observation that when traders submit demand schedules contingent on price realizations (of any subset of assets), it is useful to adopt the perspective of an individual trader who optimizes against a profile of his residual supply functions, which is the sufficient statistic of a residual market $\left\{\left\{q_{k}^{j}(\cdot)\right\}_{k}\right\}_{j \neq i \cdot}{ }^{19}$ The residual supply $S_{k}^{-i, c}(\cdot): \mathbb{R}^{K} \rightarrow \mathbb{R}$ for asset $k$ is a function of $\mathbf{p}$ if demands are contingent $\left(\left\{q_{k}^{i, c}(\cdot): \mathbb{R}^{K} \rightarrow \mathbb{R}\right\}_{i}\right)$, and $S_{k}^{-i}(\cdot): \mathbb{R} \rightarrow \mathbb{R}$ is a function of $p_{k}$ if demands are uncontingent $\left(\left\{q_{k}^{i}(\cdot): \mathbb{R} \rightarrow \mathbb{R}\right\}_{i}\right)$. Second, in equilibrium, the residual supply functions are correct: $S_{k}^{-i}(\cdot)=-\sum_{j \neq i} q_{k}^{j}(\cdot)$ for all $k$ by aggregation through market clearing of the other traders' submitted schedules.

\footnotetext{
${ }^{18}$ The common value component $\mathbf{q}_{0}^{c v}$ in traders' endowments $\left\{\mathbf{q}_{0}^{i}\right\}_{i}$ ensures that the price (equivalently, the per-capita aggregate endowment) is random in the limit large market $(I \rightarrow \infty)$. To make the price variance $\operatorname{Var}\left[\mathbf{p} \mid \mathbf{q}_{0}^{i}\right]=\mathbf{\Sigma} \operatorname{Var}\left[\left(\sum_{j} \frac{1}{\alpha^{j}}\right)^{-1} \mathbf{q}_{0}^{j} \mid \mathbf{q}_{0}^{i}\right] \mathbf{\Sigma}^{\prime}$ independent of the number of traders $I$, the risk aversion $\alpha^{i}$ in utility (1) can be scaled according to $\alpha^{i, I} \equiv \alpha^{i} \frac{I}{I-1} \sqrt{\left(\sigma_{c v}+\frac{1}{I} \sigma_{p v}\right)^{-1} \sigma_{c v}}$. As $I \rightarrow \infty, \alpha^{i, I} \rightarrow \alpha^{i}>0$ for all $i$. More generally, one can jointly scale $\left(\alpha^{i}, \sigma_{c v}, \sigma_{p v}\right)$.

${ }^{19}$ The idea of considering the pointwise optimization problem of a single trader, taking as given his residual market, goes back to Klemperer and Meyer (1989) and Kyle (1989). Rostek and Weretka (2015) introduced the equilibrium characterization in terms of the fixed point in price impacts (Proposition 1 below), showing equivalence between the equilibrium conditions in Definition 2 and Proposition 1. The characterization of the equilibrium with contingent demands for heterogeneous risk aversions (Eq. (8)) is from Malamud and Rostek (2017).
} 


\subsection{Equilibrium With Contingent Demands}

Consider the optimization problem (2) of trader $i$ who submits demand schedules $\mathbf{q}^{i, c}(\cdot): \mathbb{R}^{K} \rightarrow \mathbb{R}^{K}$ contingent on price realizations for all assets $\mathbf{p} \in \mathbb{R}^{K} \cdot{ }^{20}$ It is well known that maximizing the expected payoff (2) is the same as maximizing the ex post payoff pointwise with respect to realizations of p (e.g., Klemperer and Meyer (1989), Kyle (1989)): for each asset $k$,

$$
\max _{q_{k}^{i, c} \in \mathbb{R}}\left\{\boldsymbol{\delta} \cdot\left(\mathbf{q}^{i, c}+\mathbf{q}_{0}^{i}\right)-\frac{\alpha^{i}}{2}\left(\mathbf{q}^{i, c}+\mathbf{q}_{0}^{i}\right) \cdot \boldsymbol{\Sigma}\left(\mathbf{q}^{i, c}+\mathbf{q}_{0}^{i}\right)-\mathbf{p} \cdot \mathbf{q}^{i, c}\right\} \quad \forall \mathbf{p} \in \mathbb{R}^{K},
$$

given the trader's demands for other assets $\left\{q_{\ell}^{i, c}(\cdot)\right\}_{\ell \neq k}$ and his residual supply function $\mathbf{S}^{-i, c}(\cdot): \mathbb{R}^{K} \rightarrow \mathbb{R}^{K}$ for all assets. In essence, the equivalence follows because the demand for each asset is measurable with respect to $\left\{\mathbf{p}, \mathbf{q}_{0}^{i}\right\}$ (i.e., the contingent variable $\mathbf{p}$ and the privately known endowment vector $\mathbf{q}_{0}^{i}$ ) and, as we will show, price distribution has full support (see Remark 1).

Coupled with the requirement that the residual supply is correct, that is, $\mathbf{S}^{-i, c}(\cdot)=$ $-\sum_{j \neq i} \mathbf{q}^{j, c}(\cdot)$ for all $i$, pointwise optimization leads to an equilibrium characterization in terms of two simple conditions (Proposition 1).

Step 1 (Optimization, given price impact). The first-order condition with respect to the demand for each asset $q_{k}^{i, c}$ is: for each $k$,

$$
\underbrace{\delta_{k}-\alpha^{i}\left(\sigma_{k k}\left(q_{k}^{i, c}+q_{0, k}^{i}\right)+\sum_{\ell \neq k} \sigma_{k \ell}\left(q_{\ell}^{i, c}+q_{0, \ell}^{i}\right)\right)}_{\text {Marginal utility for asset } k}=\underbrace{p_{k}+\frac{d p_{k}}{d q_{k}^{i, c}} q_{k}^{i, c}+\sum_{\ell \neq k} \frac{d p_{\ell}}{d q_{k}^{i, c}} q_{\ell}^{i, c}}_{\text {Marginal payment for asset } k}
$$

In a linear equilibrium, ${ }^{21} \frac{d p_{\ell}}{d q_{k}^{i, c}} \equiv \lambda_{k \ell}^{i, c}$ is constant for each $k, \ell$, and $i$. Written in matrix form, the first-order conditions (3) become

$$
\boldsymbol{\delta}-\alpha^{i} \boldsymbol{\Sigma}\left(\mathbf{q}^{i, c}+\mathbf{q}_{0}^{i}\right)=\mathbf{p}+\boldsymbol{\Lambda}^{i, c} \mathbf{q}^{i, c} \quad \forall \mathbf{p} \in \mathbb{R}^{K},
$$

\footnotetext{
${ }^{20}$ A unilateral demand change of trader $i$ is understood as a profile of arbitrary twice continuously differentiable functions $\left\{\Delta q_{k}^{i}(\cdot): \mathbb{R}^{K} \rightarrow \mathbb{R}\right\}_{k}$ so that $\mathbf{q}^{i}(\cdot)+\Delta \mathbf{q}^{i}(\cdot)$ is downward-sloping with respect to $\mathbf{p} \in \mathbb{R}^{K}$, that is, the Jacobian $\frac{\partial\left(\mathbf{q}^{i}(\cdot)+\Delta \mathbf{q}^{i}(\cdot)\right)}{\partial \mathbf{p}} \in \mathbb{R}^{K \times K}$ is negative semi-definite.

${ }^{21}$ More precisely, assuming that the best-response demands of traders $j \neq i$ are linear. If utilities were nonquadratic or distributions of traders' initial holdings were non-Gaussian, the equilibrium would not be linear. While, in a nonlinear equilibrium, some conditions might be required for the one-to-one mapping between the price realizations for each asset and the residual supply intercepts' realizations of these assets, the equivalence with pointwise optimization does not hinge on the linear-quadratic environment. In a nonlinear equilibrium, additional effects would arise and accordingly amend the welfare analysis; however, the main results would apply (e.g., nonredundancy, welfare trade-off, alternative implementation of the contingent outcome). These results do not rely on the linearity, only the monotonicity between conditioning variables and random variables.
} 
where matrix

$$
\Lambda^{i, c} \equiv \frac{d \mathbf{p}}{d \mathbf{q}^{i, c}}=\left(\frac{d p_{\ell}}{d q_{k}^{i, c}}\right)_{k, \ell}=\left[\begin{array}{ccc}
\frac{d p_{1}}{d q_{1}^{i, c}} & \cdots & \frac{d p_{K}}{d q_{1}^{i, c}} \\
\vdots & \ddots & \vdots \\
\frac{d p_{1}}{d q_{K}^{i, c}} & \cdots & \frac{d p_{K}}{d q_{K}^{i, c}}
\end{array}\right] \in \mathbb{R}^{K \times K}
$$

is the price impact of trader $i$. Its $(k, \ell)$ th element $\lambda_{k \ell}^{i, c}$ represents the price change in asset $\ell$ following a demand change in asset $k$ by trader $i$. The inverse of price impact is a common measure of liquidity: the lower the price impact, the smaller the price concession a trader must accept, and the more liquid the market. From the first-order condition (4), the best-response demand of trader $i$ is

$$
\mathbf{q}^{i, c}(\mathbf{p})=\left(\alpha^{i} \boldsymbol{\Sigma}+\boldsymbol{\Lambda}^{i, c}\right)^{-1}\left(\boldsymbol{\delta}-\mathbf{p}-\alpha^{i} \boldsymbol{\Sigma} \mathbf{q}_{0}^{i}\right) \quad \forall \mathbf{p} \in \mathbb{R}^{K},
$$

given his price impact $\Lambda^{i, c}$, which is a sufficient statistic for trader $i$ 's residual supply function (see Remark 3) and is endogenized in Step 2.

Step 2 (Correct price impact). In equilibrium, the price impact in the pointwise first-order condition (4) of trader $i$ must be correct, that is, must equal the transpose of the $K \times K$ Jacobian matrix of the trader's inverse residual supply function. Applying market clearing to the best-response demands (5) for traders $j \neq i$ yields the residual supply function $\mathbf{S}^{-i, c}(\cdot)$ of trader $i$ :

$$
\mathbf{S}^{-i, c}(\mathbf{p})=-\sum_{j \neq i}\left(\alpha^{j} \boldsymbol{\Sigma}+\boldsymbol{\Lambda}^{j, c}\right)^{-1}\left(\boldsymbol{\delta}-\alpha^{j} \boldsymbol{\Sigma} \mathbf{q}_{0}^{j}\right)+\sum_{j \neq i}\left(\alpha^{j} \boldsymbol{\Sigma}+\boldsymbol{\Lambda}^{j, c}\right)^{-1} \mathbf{p} \quad \forall \mathbf{p} \in \mathbb{R}^{K} .
$$

The price impact of trader $i$ is $\Lambda^{i, c} \equiv\left(\frac{d p_{\ell}}{d q_{k}^{i c c}}\right)_{k, \ell}=\left(\left(\frac{\partial \mathbf{S}^{-i, c}(\cdot)}{\partial \mathbf{p}}\right)^{-1}\right)^{\prime}$.

Proposition 1 gives an equivalent characterization of the equilibrium in demand schedules by two conditions: (i) traders optimize, given their assumed price impacts, (ii) which are correct.

PROPOSITION 1-Equilibrium: Contingent Trading: A profile of (net) demand schedules $\left\{\mathbf{q}^{i, c}(\cdot)\right\}_{i}$ is a linear Bayesian Nash equilibrium if and only if, for each trader $i$,

(i) (Optimization, given price impact) Demand schedules $\mathbf{q}^{i, c}(\cdot): \mathbb{R}^{K} \rightarrow \mathbb{R}^{K}$ are determined by pointwise equalization of marginal utility and marginal payment in Eq. (5), given his price impact $\boldsymbol{\Lambda}^{i, c}$, such that:

(ii) (Correct price impact) The price impact of trader $i$ equals the transpose of the Jacobian of his inverse residual supply function:

$$
\boldsymbol{\Lambda}^{i, c}=\left(\left(\sum_{j \neq i}\left(\alpha^{j} \mathbf{\Sigma}+\boldsymbol{\Lambda}^{j, c}\right)^{-1}\right)^{-1}\right)^{\prime} .
$$

The fixed point for price impact matrices defined by the system of $I$ equations (7) can be solved in closed form when demands are contingent: for each $i$,

$$
\Lambda^{i, c}=\beta^{i, c} \alpha^{i} \Sigma
$$


where $\beta^{i, c}=\frac{2-\alpha^{i} b+\sqrt{\left(\alpha^{i} b\right)^{2}+4}}{2 \alpha^{i} b} \in \mathbb{R}_{+}$and $b \in \mathbb{R}_{+}$is the unique solution to $\sum_{j}\left(\alpha^{j} b+2+\right.$ $\left.\sqrt{\left(\alpha^{j} b\right)^{2}+4}\right)^{-1}=1 / 2$. If risk aversions are symmetric (i.e., $\alpha^{i}=\alpha$ for all $i$ ), then the price impact is $\boldsymbol{\Lambda}^{i, c}=\frac{\alpha}{I-2} \mathbf{\Sigma}$.

Analyzing the price impact directly offers insights into the role of imperfectly competitive behavior. As $I \rightarrow \infty$, then $\boldsymbol{\Lambda}^{i, c} \rightarrow \mathbf{0}$ for all $i,{ }^{22}$ and the competitive limit demand coincides with the inverse marginal utility, given the quasilinearity of the payoff function. When the price impact is positive, $\boldsymbol{\Lambda}^{i, c}>\mathbf{0}$, trader $i$ demands (or sells) less than if he had submitted his competitive schedule.

Remarks. We note four properties, which—with the exception of the second-do not hold when demands are not contingent.

1. By Eq. (8), the price impact of trader $i$ derives from the utility concavity of the residual market $\left\{\alpha^{j} \mathbf{\Sigma}\right\}_{j \neq i}{ }^{23}$ and, with contingent trading, the equilibrium price impact of every trader is proportional to the fundamental covariance matrix $\mathbf{\Sigma}$. This proportionality between incentives $(\boldsymbol{\Lambda})$ and fundamental risk $(\boldsymbol{\Sigma})$ has important implications for how the contingent market functions and, as we will show, does not hold in more general designs because of cross-asset inference (cf. Theorem 3, Proposition S2 in Appendix C.1).

2. All price realizations $\mathbf{p} \in \mathbb{R}^{K}$ occur in equilibrium for some realizations of endowments, given the traders' downward-sloping demands (i.e., the Jacobian $\frac{\partial \mathbf{q}^{i, c} \cdot(\cdot)}{\partial \mathbf{p}}=-\left(\alpha^{i} \mathbf{\Sigma}+\right.$ $\left.\left.\boldsymbol{\Lambda}^{i}\right)^{-1}<\mathbf{0}\right){ }^{24}$ The market-clearing condition (Definition 2) is accounted for by condition (ii) for price impacts (Eq. (7)). Hence, the first-order conditions must hold for all prices, and the price impact of each trader is determined by the requirement that optimization, Bayesian inference, and market clearing hold in equilibrium and following a unilateral demand change. This makes precise the difference with Nash-in-Nash (see Introduction).

3. A trader's own price impact $\boldsymbol{\Lambda}^{i, c}$ is a sufficient statistic for the residual supply function in the best-response problem. This holds due to the one-to-one mapping between the contingent variable (i.e., price vector $\mathbf{p}$ ) and the residual supply's intercept (i.e., the vector $\mathbf{s}^{-i, c} \equiv-\sum_{j \neq i}\left(\alpha^{j} \mathbf{\Sigma}+\Lambda^{j, c}\right)^{-1}\left(\boldsymbol{\delta}-\alpha^{j} \mathbf{\Sigma} \mathbf{q}_{0}^{j}\right) \in \mathbb{R}^{K}$ in Eq. (6)) for all assets.

4. Equilibrium is ex post, given the one-to-one mapping.

\subsection{Equilibrium With Uncontingent Demands}

Consider the optimization problem (2) of trader $i$ in a market with $K$ exchanges, each for one asset, who submits demand schedules $\left\{q_{k}^{i}(\cdot): \mathbb{R} \rightarrow \mathbb{R}\right\}_{k} \cdot{ }^{25}$ The trader's objective function is the same as with contingent trading; in particular, his information set (i.e., $\mathbf{q}_{0}^{i}$ ) is. However, the choice variable differs: the demand in the exchange for asset $k$ is contingent on, and hence measurable with respect to, price $p_{k}$ only. Consequently, maximizing expected payoff (2) is not the same as maximizing ex post payoff.

\footnotetext{
${ }^{22}$ The price impact converges to zero as $I \rightarrow \infty$ so long as the risk aversion $\alpha^{i, I}$ increases slower than linearly, $\alpha^{i, I}=\alpha^{i} \gamma^{I}$ where $\gamma^{I} \sim o\left(I^{1-\varepsilon}\right)$ for some $\varepsilon \in(0,1)$ (Lemma S3 in Appendix B). Aggregate endowment is random in the limit, provided that traders' endowments are correlated via $\mathbf{q}_{0}^{c v}\left(\sigma_{c v}>0\right)$; see ft. 18.

${ }^{23}$ The riskier the assets traded and the more risk averse trader $i$ 's counterparties, the less elastic their marginal utilities, the less elastic the residual supply of trader $i$, and the larger the price concessions that $i$ has to accept.

${ }^{24}$ If $\mathbf{M}$ is not invertible, $\mathbf{M}^{-1}$ is the Moore-Penrose pseudoinverse of $\mathbf{M}$.

${ }^{25} \mathrm{~A}$ unilateral demand change of trader $i$ is understood as a profile of arbitrary twice continuously differentiable functions $\left\{\Delta q_{k}^{i}(\cdot): \mathbb{R} \rightarrow \mathbb{R}\right\}_{k}$ so that $q_{k}^{i}(\cdot)+\Delta q_{k}^{i}(\cdot)$ is downward-sloping, that is, $\frac{\partial\left(q_{k}^{i}(\cdot)+\Delta q_{k}^{i}(\cdot)\right)}{\partial p_{k}}<0$ for all $k$.
} 
Proposition 2 establishes that, analogously to the contingent market (Proposition 1), a trader's pointwise optimization for each asset $k$, now with respect to $p_{k} \in \mathbb{R}$, is necessary and sufficient for optimization in demand functions (i.e., $\left.\left\{q_{k}^{i}(\cdot)\right\}_{k}\right)$ : for each asset $k$,

$$
\max _{q_{k}^{i} \in \mathbb{R}} E\left[\boldsymbol{\delta} \cdot\left(\mathbf{q}^{i}+\mathbf{q}_{0}^{i}\right)-\frac{\alpha^{i}}{2}\left(\mathbf{q}^{i}+\mathbf{q}_{0}^{i}\right) \cdot \boldsymbol{\Sigma}\left(\mathbf{q}^{i}+\mathbf{q}_{0}^{i}\right)-\mathbf{p} \cdot \mathbf{q}^{i} \mid p_{k}, \mathbf{q}_{0}^{i}\right] \quad \forall p_{k} \in \mathbb{R},
$$

given his demands for other assets $\left\{q_{\ell}^{i}(\cdot)\right\}_{\ell \neq k}$ and a profile of residual supply functions $\left\{S_{\ell}^{-i}(\cdot): \mathbb{R} \rightarrow \mathbb{R}\right\}_{\ell}$ for all assets.

Compared to contingent trading (Eq. (3)), the first-order condition differs in two ways:

$$
\underbrace{\delta_{k}-\alpha^{i}\left(\sigma_{k k}\left(q_{k}^{i}+q_{0, k}^{i}\right)+\sum_{\ell \neq k} \sigma_{k \ell}(\underbrace{E\left[q_{\ell}^{i} \mid p_{k}, \mathbf{q}_{0}^{i}\right]}_{\begin{array}{c}
\text { Expected trade } \\
\text { of asset } \ell
\end{array}}+q_{0, \ell}^{i})\right)}_{\text {Expected marginal utility for asset } k}=\underbrace{p_{k}+\underbrace{\lambda_{k}^{i}}_{\begin{array}{c}
\text { Zero cross-exchange } \\
\text { priment }
\end{array}} q_{k}^{i}}_{\begin{array}{c}
\text { Marginal payment for asset } k \\
\text { price impact }
\end{array}}
$$

$$
\forall p_{k} \in \mathbb{R},
$$

where $\lambda_{k}^{i} \equiv \frac{d p_{k}}{d q_{k}^{i}} \in \mathbb{R}_{+}$is the price impact of trader $i$ in the exchange for asset $k$; in a linear equilibrium, $\lambda_{k}^{i}$ is constant. First, a trader's demand for asset $k$ depends on expected rather than realized trades of other assets $\ell \neq k, E\left[q_{\ell}^{i} \mid p_{k}, \mathbf{q}_{0}^{i}\right]$. Equilibrium is generally not ex post. Second, the cross-exchange price impact is zero: $\lambda_{k \ell}^{i} \equiv \frac{d p_{\ell}}{d q_{k}^{i}}=0$ for all $k$ and $\ell \neq k$, since the residual supply function $S_{k}^{-i}\left(\cdot ;\left\{\mathbf{q}_{0}^{j}\right\}_{j \neq i}\right): \mathbb{R} \rightarrow \mathbb{R}$ is contingent on $p_{k}$ but not $\left\{p_{\ell}\right\}_{\ell \neq k}$. It follows that, in contrast to the contingent market, where the price impact matrices of all traders are proportional to the covariance matrix $\mathbf{\Sigma}$ (Eq. (8) and Remark 1), the price impacts of all traders are diagonal matrices: for each $i$,

$$
\Lambda^{i} \equiv\left(\frac{d p_{\ell}}{d q_{k}^{i}}\right)_{k, \ell}=\operatorname{diag}\left(\lambda_{k}^{i}\right)_{k} \in \mathbb{R}^{K \times K} .
$$

Although the cross-exchange price impact is eliminated, equilibrium behavior and outcome (i.e., prices and allocations) are not independent across exchanges-unless all assets' payoffs are independent (i.e., $\sigma_{k \ell}=0$ for all $\ell \neq k$ ), in which case traders' utility Hessian is separable.

Proposition 2 takes the intercept of trader $i$ 's residual supply $s_{k}^{-i}$ rather than price $p_{k}$ as a contingent variable $-s_{k}^{-i}$ is exogenous in the best-response problem of trader $i$. This allows us to separate the best-response and equilibrium problems analogously to Proposition 1: (i) optimization by trader $i$, given $i$ 's residual supply (Step 1); (ii) which is correct (Step 2).

For each trader $i$, let $F\left(\left(\mathbf{q}_{0}^{j}\right)_{j \neq i} \mid \mathbf{q}_{0}^{i}\right)$ be the joint distribution of other traders' endowments and let $F\left(\mathbf{s}^{-i} \mid \mathbf{q}_{0}^{i}\right)$ be the joint distribution of the intercepts $\mathbf{s}^{-i} \equiv\left(s_{k}^{-i}\right)_{k}$ of the residual supplies of trader $i$-both conditional on trader $i$ 's privately known endowment. The former distribution is a primitive object; the latter is not, but it is taken as given in trader $i$ 's best-response problem. Given the linear demands $\left\{\mathbf{q}^{j}(\cdot)\right\}_{j \neq i}, F\left(\mathbf{s}^{-i} \mid \mathbf{q}_{0}^{i}\right)$ is jointly Normal.

PROPOSITION 2-Equilibrium: Uncontingent Trading: A profile of (net) demand schedules $\left\{\left\{q_{k}^{i}(\cdot)\right\}_{k}\right\}_{i}$ is a linear Bayesian Nash equilibrium if and only if, for each trader $i$, 
(i) (Optimization, given residual supply) Demand schedules $q_{k}^{i}(\cdot): \mathbb{R} \rightarrow \mathbb{R}$ are determined by equalization of expected marginal utility and marginal payment for each asset $k$ pointwise to $p_{k} \in \mathbb{R}$ :

$$
\delta_{k}-\alpha^{i} \boldsymbol{\Sigma}_{k} E\left[\mathbf{q}^{i}+\mathbf{q}_{0}^{i} \mid s_{k}^{-i}, \mathbf{q}_{0}^{i}\right]=p_{k}+\lambda_{k}^{i} q_{k}^{i} \quad \forall p_{k} \in \mathbb{R},
$$

given the trader's own demands for other assets $\left\{q_{\ell}^{i}(\cdot)\right\}_{\ell \neq k}$, the distribution $F\left(\mathbf{s}^{-i} \mid \mathbf{q}_{0}^{i}\right)$, and price impact $\boldsymbol{\Lambda}^{i}=\operatorname{diag}\left(\lambda_{k}^{i}\right)_{k}$.

(ii) (Correct residual supply) The residual supply function $S_{k}^{-i}(\cdot): \mathbb{R} \rightarrow \mathbb{R}$ of trader $i$ is determined by applying market clearing to the best responses of traders $j \neq i\left\{q_{k}^{j}(\cdot)\right\}_{j \neq i}$ that satisfy condition ( $i)$ : for each $k$,

$$
S_{k}^{-i}(\cdot)=-\sum_{j \neq i} q_{k}^{j}(\cdot) .
$$

The price impact $\lambda_{k}^{i}$ of trader $i$ is characterized by the slope of $\left(S_{k}^{-i}(\cdot)\right)^{-1}$. The distribution $F\left(\mathbf{s}^{-i} \mid \mathbf{q}_{0}^{i}\right)$ is characterized by the intercept of $S_{k}^{-i}(\cdot)$, given $F\left(\left(\mathbf{q}_{0}^{j}\right)_{j \neq i} \mid \mathbf{q}_{0}^{i}\right)$.

Remarks.

5 . Because the demand for asset $k$ is not measurable with respect to prices of other assets, asset by asset optimization and the equivalence with the pointwise problem is more subtle than in the contingent model. Lemma S2 in Appendix B shows that asset by asset optimization is without loss of generality by the Fréchet differentiability of expected payoff (2) with respect to the profile of demand schedules $\left\{q_{k}^{i}(\cdot)\right\}_{k}$. Proposition 2 shows that the first-order condition computed pointwise to its contingent variables in each exchange is necessary and sufficient, subject to the second-order conditions.

6. Because of inference across assets as well as across traders, the equilibrium characterization is more challenging in two ways.

First, the price impact $\Lambda^{i}$ is not by itself a sufficient statistic for the residual supply of trader $i$ (cf. Remark 3) - the joint distribution of the conditioning variable $\mathbf{s}^{-i}$ (equivalently, p) matters. Second, in a trader's best response (10) for asset $k$, expected trades $E\left[q_{\ell}^{i} \mid p_{k}, \mathbf{q}_{0}^{i}\right]$ depend on the distribution of his endogenous quantity traded of other assets, $\left\{q_{\ell}^{i}\right\}_{\ell \neq k}$. Therefore, characterizing a trader's own best-response demands requires solving a fixed point for the trader's own demand coefficients across assets.

To elaborate on the latter point, in a trader's multivariate optimization problem (2) contingent or uncontingent- the system of first-order conditions defines a fixed point problem among the trader's best-response schedules. With contingent demands, the system can be written as a single matrix equation, $\boldsymbol{\delta}-\alpha^{i} \mathbf{\Sigma}\left(\mathbf{q}^{i, c}+\mathbf{q}_{0}^{i}\right)=\mathbf{p}+\boldsymbol{\Lambda}^{i, c} \mathbf{q}^{i, c}$ for all $\mathbf{p} \in \mathbb{R}^{K}$ (Eq. (4)) for a quantity vector $\mathbf{q}^{i, c}=\left(q_{k}^{i, c}\right)_{k}$ as a function of the common conditioning variable $\mathbf{p}$, and can be solved for $\mathbf{q}^{i, c}$ pointwise with respect to $\mathbf{p}$. (Equation (5) gives the closed-form solution.) Thus, the coefficients of the best-response functions need not be characterized as a fixed point across assets.

Theorem 1 in Section 3.2.1 endogenizes all demand coefficients (Step 1)—including expected trades $\left\{\left\{E\left[q_{\ell}^{i} \mid p_{k}, \mathbf{q}_{0}^{i}\right]\right\}_{\ell \neq k}\right\}_{k}$ —and the distribution of the residual supply (Step 2) as functions of price impacts $\left\{\boldsymbol{\Lambda}^{i}\right\}_{i}$. It thus shows that a fixed point in uncontingent demand schedules $\left\{\left\{q_{k}^{i}(\cdot)\right\}_{k}\right\}_{i}$ is equivalent to a fixed point in price impact matrices.

\subsubsection{Equilibrium as a Fixed Point in Price Impacts}

This section presents our main characterization result, Theorem 1 . To tackle the characterization of the fixed point problem for a trader's best-response schedules $\left\{q_{k}^{i}(\cdot)\right\}_{k}$ across 
assets, we first transform the system of first-order conditions (10) into a fixed point among the trader's demand coefficients, given the residual supplies, that is, $\Lambda^{i}$ and $F\left(\mathbf{s}^{-i} \mid \mathbf{q}_{0}^{i}\right)$ (Step 1). We then endogenize the distribution of the residual supply-and thus all demand coefficients, including expected trades $E\left[q_{\ell}^{i} \mid p_{k}, \mathbf{q}_{0}^{i}\right]$ for all $\ell \neq k$ and $k$-as a function of price impacts $\left\{\boldsymbol{\Lambda}^{i}\right\}_{i}$ and characterize equilibrium as the fixed point for $\left\{\boldsymbol{\Lambda}^{i}\right\}_{i}$ (Step 2).

Best-response problem (Step 1). In each exchange $k$, we parameterize a trader's conjectured best responses for other assets $\ell \neq k$ as linear functions of $p_{\ell}$ and $\mathbf{q}_{0}^{i}$ :

$$
q_{\ell}^{i}\left(p_{\ell}\right) \equiv a_{\ell}^{i}-\mathbf{b}_{\ell}^{i} \mathbf{q}_{0}^{i}-c_{\ell}^{i} p_{\ell} \quad \forall p_{\ell} \in \mathbb{R},
$$

with the demand intercept $a_{\ell}^{i} \in \mathbb{R}$, the demand coefficients $\mathbf{b}_{\ell}^{i} \in \mathbb{R}^{1 \times K}$ on $\mathbf{q}_{0}^{i}$, and the demand slope $c_{\ell}^{i} \in \mathbb{R}_{+}$on $p_{\ell}$.

The parameterization of demands (12) allows us to change the conditioning variables in expected trades (from $p_{k}$ to $s_{k}^{-i}$ ) and endogenize expected trades in terms of variables that are exogenous in the trader's best-response problem for asset $k$. By market clearing, given the residual supply $S_{\ell}^{-i}(\cdot)=s_{\ell}^{-i}+\left(\lambda_{\ell}^{i}\right)^{-1} p_{\ell}$, we have $a_{\ell}^{i}-\mathbf{b}_{\ell}^{i} \mathbf{q}_{0}^{i}-c_{\ell}^{i} p_{\ell}=s_{\ell}^{-i}+\left(\lambda_{\ell}^{i}\right)^{-1} p_{\ell}$ for all $s_{\ell}^{-i} \in \mathbb{R}$, which gives $p_{\ell}$ as a linear function of $s_{\ell}^{-i}$ and

$$
E\left[q_{\ell}^{i} \mid p_{k}, \mathbf{q}_{0}^{i}\right]=E\left[a_{\ell}^{i}-\mathbf{b}_{\ell}^{i} \mathbf{q}_{0}^{i}-\frac{c_{\ell}^{i}}{c_{\ell}^{i}+\left(\lambda_{\ell}^{i}\right)^{-1}}\left(a_{\ell}^{i}-\mathbf{b}_{\ell}^{i} \mathbf{q}_{0}^{i}-s_{\ell}^{-i}\right) \mid s_{k}^{-i}, \mathbf{q}_{0}^{i}\right] \quad \forall s_{k}^{-i} \in \mathbb{R}
$$

Theorem 1 shows that when traders' risk aversions are the same, the fixed point problem for the coefficients of best-response schedules $\left\{q_{k}^{i}(\cdot)\right\}_{k}$ in Eqs. (13)-(15) has a unique solution.

Equilibrium as a fixed point in price impacts (Step 2). With best-response coefficients $\left\{a_{k}^{i}, \mathbf{b}_{k}^{i}, c_{k}^{i}\right\}_{k}$ endogenized as functions of $\Lambda^{i}$ and $F\left(\mathbf{s}^{-i} \mid \mathbf{q}_{0}^{i}\right)$, the equilibrium fixed point problem becomes one among the traders' residual supply functions $\left\{\Lambda^{i}, F\left(\mathbf{s}^{-i} \mid \mathbf{q}_{0}^{i}\right)\right\}_{i}$. The latter fixed point problem across traders is still complex and has a larger dimensionality. ${ }^{26}$

Nevertheless, the equilibrium distributions of the residual supply $\left\{F\left(\mathbf{s}^{-i} \mid \mathbf{q}_{0}^{i}\right)\right\}_{i}$ can themselves be characterized as functions of only price impacts $\left\{\Lambda^{i}\right\}_{i}$, given the primitive distribution of endowments: Applying market clearing to the best-response schedules $\left\{q_{k}^{j}(\cdot)\right\}_{j \neq i}$ gives the residual supply functions of trader $i$ (i.e., condition (ii) in Proposition 2): for each $k$,

$$
S_{k}^{-i}\left(p_{k}\right)=\underbrace{-\sum_{j \neq i}\left(a_{k}^{j}-\mathbf{b}_{k}^{j} \mathbf{q}_{0}^{j}\right)}_{=s_{k}^{-i}}+\underbrace{\sum_{j \neq i} c_{k}^{j}}_{=\left(\lambda_{k}^{i}\right)^{-1}} p_{k} \quad \forall p_{k} \in \mathbb{R} .
$$

\footnotetext{
${ }^{26}$ In the contingent market, the equilibrium fixed point equations (i.e., price impact equations) reduce to scalar equations, given the proportionality of the price impact matrix in the covariance; hence, the fixed point problem involves $I$ scalar variables $\left\{\beta^{i, c}\right\}_{i}$ (Eq. (8)). When schedules are not contingent, since the price impact $\boldsymbol{\Lambda}^{i}$ is not by itself a sufficient statistic for the residual supply, the additional fixed point problem among the distributions of the residual supplies' intercepts of all assets involves $\left(K+\frac{K(K+1)}{2}\right) I$ variables $-K$ first moments $\left\{E\left[s_{k}^{-i} \mid \mathbf{q}_{0}^{i}\right]\right\}_{k}$ and $\frac{K(K+1)}{2}$ second moments $\left\{\operatorname{Cov}\left[s_{k}^{-i}, s_{\ell}^{-i} \mid \mathbf{q}_{0}^{i}\right]\right\}_{k, \ell}$ for each $i$. Theorem 1 shows that equilibrium can be characterized by $I K$ price impacts $\left\{\left\{\lambda_{k}^{i}\right\}_{k}\right\}_{i}$.
} 
Finally, in each exchange, the equilibrium price impact $\lambda_{k}^{i} \equiv \frac{d p_{k}}{d q_{k}^{i}} \in \mathbb{R}_{+}$must equal the slope of the inverse residual supply function: $\lambda_{k}^{i}=-\left(\sum_{j \neq i} \frac{\partial q_{k}^{j}(\cdot)}{\partial p_{k}}\right)^{-1}=\left(\sum_{j \neq i} c_{k}^{j}\right)^{-1}$ for all $i$ and $k$.

Theorem 1 characterizes the equilibrium demand coefficients $\boldsymbol{a}^{i} \equiv\left(a_{k}^{i}\right)_{k} \in \mathbb{R}^{K}, \mathbf{B}^{i} \equiv$ $\left(\mathbf{b}_{k}^{i}\right)_{k} \in \mathbb{R}^{K \times K}$, and $\mathbf{C}^{i} \equiv \operatorname{diag}\left(c_{k}^{i}\right)_{k} \in \mathbb{R}^{K \times K}$ as functions of price impact-in matrix closed form-and characterizes the equilibrium price impact in terms of primitives. In the main text, we assume symmetric risk preferences and present the characterization of the symmetric equilibrium ${ }^{27}$ for simplicity of notation. In Appendix A, we state and prove the result for an asymmetric equilibrium.

Assumption (Symmetric Risk Preferences). Let $\alpha^{i}=\alpha$ for all $i$.

Notation. Let $[\cdot]_{d}: \mathbb{R}^{K \times K} \rightarrow \mathbb{R}^{K \times K}$ be an operator such that, for any matrix $\mathbf{M},[\mathbf{M}]_{d}$ is a diagonal matrix with the $(k, \ell)$ th element equal to zero for $k \neq \ell$ and the $(k, k)$ th element equal to $m_{k k}$ for any $k$.

THEOREM 1-Equilibrium: Fixed Point in Demand Schedules: In a symmetric equilibrium, the (net) demand schedules, defined by matrix coefficients $\left\{\boldsymbol{a}^{i}\right\}_{i}, \mathbf{B}$, and $\mathbf{C}$, and price impact $\boldsymbol{\Lambda}$ are characterized by the following conditions: for each trader $i$,

(i) (Demand coefficients, given price impact) Given price impact matrix $\mathbf{\Lambda}$, the coefficients of (net) demands $\boldsymbol{a}^{i}, \mathbf{B}$, and $\mathbf{C}$ are characterized by

$$
\begin{aligned}
& \boldsymbol{a}^{i}=\mathbf{C} \underbrace{\left.\mathbf{C}-\left(\alpha \boldsymbol{\Sigma}-\mathbf{C}^{-1} \mathbf{B}\right) E\left[\overline{\mathbf{q}}_{0}\right]\right)}_{=\mathbf{p}-\mathbf{C}^{-1} \mathbf{B} \overline{\mathbf{q}}_{0}} \\
& +\left((\alpha \mathbf{\Sigma}+\boldsymbol{\Lambda})^{-1} \alpha \mathbf{\Sigma}-\mathbf{B}\right)\left(E\left[\overline{\mathbf{q}}_{0}\right]-E\left[\mathbf{q}_{0}^{i}\right]\right), \\
& \text { Adjustment due to cross-asset inference } \\
& \mathbf{B}=\left(\left(1-\sigma_{0}^{2}\right)(\alpha \mathbf{\Sigma}+\boldsymbol{\Lambda})+\sigma_{0}^{2} \mathbf{C}^{-1} \quad\right)^{-1} \alpha \mathbf{\Sigma}, \\
& \text { Adjustment due to } \\
& \text { cross-asset inference }
\end{aligned}
$$

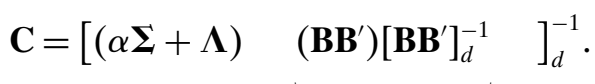

$$
\begin{aligned}
& \text { Inference coefficient } \\
& \operatorname{Var}\left[\mathbf{s}^{-i} \mid \mathbf{q}_{0}^{i}\right]\left[\operatorname{Var}\left[\mathbf{s}^{-i} \mid \mathbf{q}_{0}^{i}\right]\right]_{d}^{-1}
\end{aligned}
$$

(ii) (Correct price impact) Price impact $\boldsymbol{\Lambda}$ equals the transpose of the Jacobian of the trader's inverse residual supply function:

$$
\Lambda=\frac{1}{I-1}\left(\mathbf{C}^{-1}\right)^{\prime}=\frac{\alpha}{I-2}\left[\mathbf{\Sigma}\left(\mathbf{B B}^{\prime}\right)\left[\mathbf{B B}^{\prime}\right]_{d}^{-1}\right]_{d} .
$$

\footnotetext{
${ }^{27}$ Equilibrium is symmetric if, for all $k$, price impacts satisfy $\lambda_{k}^{i} \equiv \lambda_{k}$ for all $i$, demand coefficients satisfy $c_{k}^{i} \equiv c_{k}$ and $\mathbf{b}_{k}^{i} \equiv \mathbf{b}_{k}$ for all $i$, and $a_{k}^{i}$ is a symmetric function of $\left\{\left\{E\left[\mathbf{q}_{0}^{j}\right]\right\}_{j \neq i}, E\left[\mathbf{q}_{0}^{i}\right]\right\}$ across traders. We will suppress the superscript $i$ except where it is helpful.
} 
Note that the price slope $\mathbf{C}$ is a diagonal matrix in the uncontingent market and $\left(\mathbf{B B}^{\prime}\right)\left[\mathbf{B B}^{\prime}\right]_{d}^{-1}=\operatorname{Var}\left[\mathbf{s}^{-i} \mid \mathbf{q}_{0}^{i}\right]\left[\operatorname{Var}\left[\mathbf{s}^{-i} \mid \mathbf{q}_{0}^{i}\right]\right]_{d}^{-1}$ is the inference coefficient. Appendix C.2 derives demand coefficients for $K=2$.

Equilibrium outcome. Theorem 1 enables a direct comparison between the imperfectly competitive $(I<\infty)$ and competitive $(I \rightarrow \infty)$ outcomes. The competitive case is characterized by $\boldsymbol{\Lambda}^{i} \rightarrow \mathbf{0}$ for all $i$.

By Theorem 1, the implications of independence in market clearing that we will subsequently characterize can be understood through the structure of the endogenous price impact matrix. To begin, Corollary 1 shows how independence in market clearing affects the equilibrium outcome. To ease the comparison, if the market clears jointly, then by Eqs. (5) and (8),

$$
\mathbf{B}^{c}=\left(\alpha \boldsymbol{\Sigma}+\boldsymbol{\Lambda}^{c}\right)^{-1} \alpha \boldsymbol{\Sigma}=\frac{I-2}{I-1} \mathbf{I d}, \quad \mathbf{C}^{c}=\left(\alpha \boldsymbol{\Sigma}+\boldsymbol{\Lambda}^{c}\right)^{-1}, \quad \mathbf{\Lambda}^{c}=\frac{\alpha}{I-2} \boldsymbol{\Sigma},
$$

where $\mathbf{I d} \in \mathbb{R}^{K \times K}$ is the identity matrix. By contrast, in the uncontingent market, traders' demand coefficients depend on the distribution of traders' endowments through crossasset inference, as do price impacts.

COROLlARY 1-Equilibrium Prices and Allocations: Given the equilibrium demand coefficients $\left\{\boldsymbol{a}^{i}\right\}_{i}, \mathbf{B}, \mathbf{C}$, and price impact $\boldsymbol{\Lambda}$ in Theorem 1, equilibrium prices and allocations are

$$
\begin{aligned}
\mathbf{p} & =\boldsymbol{\delta}-\left(\alpha \boldsymbol{\Sigma}-\mathbf{C}^{-1} \mathbf{B}\right) E\left[\overline{\mathbf{q}}_{0}\right]-\mathbf{C}^{-1} \mathbf{B} \overline{\mathbf{q}}_{0}, \\
\mathbf{q}^{i}+\mathbf{q}_{0}^{i} & =\left((\alpha \boldsymbol{\Sigma}+\boldsymbol{\Lambda})^{-1} \alpha \boldsymbol{\Sigma}-\mathbf{B}\right)\left(E\left[\overline{\mathbf{q}}_{0}\right]-E\left[\mathbf{q}_{0}^{i}\right]\right)+\mathbf{B} \overline{\mathbf{q}}_{0}+(\mathbf{I d}-\mathbf{B}) \mathbf{q}_{0}^{i} .
\end{aligned}
$$

We highlight two implications of the outcome's dependence on inference. In contrast to the contingent market, where $\mathbf{p}^{c}=\boldsymbol{\delta}-\alpha \mathbf{\Sigma} \overline{\mathbf{q}}_{0}$, the second moment $\operatorname{Var}[\mathbf{p}]$ of the distribution of equilibrium prices depends on the distribution of endowments-through the endogenous demand coefficient $\mathbf{C}^{-1} \mathbf{B}$-rather than only the exogenous asset covariance $\mathbf{\Sigma}$. In particular, the price covariance of any asset pair depends on the second moment of the joint distribution of all assets and not merely the covariance of the pair. (We explore the implications of this property in Section 3.2.3 and Example 3.) Additionally, the allocations' weights on the idiosyncratic and market risk (i.e., Id $-\mathbf{B}$ and $\mathbf{B}$ ) now depend on the asset covariance and the distribution of endowments. Thus, asset payoff substitution $(\Sigma)$ itself factors in which assets' allocation is more efficient.

Intuitively, cross-asset inference changes how traders' incentives $(\boldsymbol{\Lambda})$ are shaped by the fundamental risk $(\boldsymbol{\Sigma})$. More precisely, independence in market clearing breaks the proportionality between price impact, and thus cross-asset demand substitution (i.e., the inverse of Jacobian $\mathbf{C}$ ), and asset covariance (cf. Remark 1). The nonproportionality of $\mathbf{C}$ to $\Sigma^{-1}$ continues to hold in the limit as $I \rightarrow \infty$.

THEOREM 2-Existence of Symmetric Equilibrium: There exists a symmetric linear Bayesian Nash equilibrium. When $K=2$, equilibrium is unique.

In the contingent market, the proportionality of price impact to asset covariance reduces the fixed point problem for $\left\{\Lambda^{i, c}\right\}_{i}$ to one for scalars $\left\{\beta^{i, c} \in \mathbb{R}\right\}_{i}$ (Eq. (8)). In the uncontingent market, the argument differs in two ways, due to cross-asset inference (i.e., inference coefficient $\left.\left(\mathbf{B B}^{\prime}\right)\left[\mathbf{B B}^{\prime}\right]_{d}^{-1}\right)$. First, price impact matrices are not proportional to 
the covariance, and ought to be found jointly for all assets and traders. Second, the mapping for price impact $\Lambda^{i}$-defined by the fixed point Eqs. (14) and (16) - is not monotone in price impacts $\left\{\boldsymbol{\Lambda}^{j}\right\}_{j \neq i}$.

Given Theorem 1's result that a fixed point in demand schedules can equivalently (for $I<\infty)$ be represented as a fixed point in price impact matrices, the existence of equilibrium follows from the Brouwer fixed point theorem (Theorem 2) with the bounds on the price impact being matrices (rather than scalars). ${ }^{28}$

\subsubsection{Preview}

Example 1 provides a preview of the results that follow.

EXAMPLE 1-Price Impact With Uncontingent Demands: Consider a market with two imperfectly correlated assets, $0<\left|\rho_{12}\right|<1, \rho_{12}=\frac{\sigma_{12}}{\sqrt{\sigma_{11} \sigma_{22}}}$ :

$$
\boldsymbol{\Sigma}=\left[\begin{array}{ll}
\sigma_{11} & \sigma_{12} \\
\sigma_{21} & \sigma_{22}
\end{array}\right], \quad \boldsymbol{\Lambda}^{i, c}=\left[\begin{array}{cc}
\lambda_{1}^{i, c} & \lambda_{12}^{i, c} \\
\lambda_{21}^{i, c} & \lambda_{2}^{i, c}
\end{array}\right], \quad \boldsymbol{\Lambda}^{i}=\left[\begin{array}{cc}
\lambda_{1}^{i} & 0 \\
0 & \lambda_{2}^{i}
\end{array}\right] .
$$

In the contingent market, the cross-asset price impact inherits the covariance's sign, by the proportionality of the price impact $\Lambda^{i, c}$ in the covariance $\boldsymbol{\Sigma}$ (Eq. (8) and Remark 1). When the assets are payoff substitutes, $\sigma_{12}>0$, for the traders who take the same (buying or selling) position in both assets, the cross-asset price impacts $\lambda_{12}^{i, c}>0$ and $\lambda_{21}^{i, c}>0$ increase the marginal trading cost of each asset:

$$
p_{1}+\lambda_{1}^{i, c} q_{1}^{i, c}+\lambda_{12}^{i, c} q_{2}^{i, c}, \quad p_{2}+\lambda_{2}^{i, c} q_{2}^{i, c}+\lambda_{21}^{i, c} q_{1}^{i, c},
$$

thereby exacerbating the demand reduction relative to the competitive demand. When the assets are payoff complements, $\sigma_{12}<0$, the negative cross-asset price impacts $\lambda_{12}^{i, c}<0$ and $\lambda_{21}^{i, c}<0$ lower the trading costs. These effects are absent with uncontingent demands-the cross-asset price impacts $\lambda_{12}^{i}$ and $\lambda_{21}^{i}$ are zero. Moreover, as we will show, the withinexchange price impacts $\lambda_{1}^{i}$ and $\lambda_{2}^{i}$ change due to cross-asset inference (Theorem 3 ).

As Example 1 indicates (and Proposition 3 and Corollary 4 will demonstrate), letting assets clear independently can increase welfare in some trading environments. Unlike the competitive market, the characteristics of traders (i.e., trading needs across assets) and assets (i.e., the asset covariance) matter for which design is efficient. In particular, when traders have an impact on prices, neither the market structure in which all assets clear jointly nor that in which each asset is traded in a separate exchange is always efficient.

\footnotetext{
${ }^{28}$ Proposition $\mathrm{S} 1$ in Appendix C provides a uniqueness result for $K \geq 2$ in symmetric markets. In more general markets, the numerical iteration that solves the equilibrium fixed point equation (Theorem 1 ) converges to the same equilibrium in extensive simulations including random initial values, different forms of the fixed point equation, and fixed points with respect to $\boldsymbol{\Lambda}$ and $\mathbf{C}$. In the contingent model, the equilibrium uniqueness can be shown outside the symmetric-market environments by applying the argument from a one-asset market, using the proportionality of the price impact in the covariance matrix (Eq. (8)) (see Malamud and Rostek (2017)). Lambert, Ostrovsky, and Panov (2018) considered a game in which strategies are quantities (market orders) with one asset and one liquidity provider; the scalar price impact solves a quadratic equation that has a unique positive solution, which gives equilibrium uniqueness. We analyze games in demand and supply functions with multiple assets and price impacts characterized by a system of nonlinear (non-polynomial) matrix equations.
} 
We will show that one can design a market with multiple venues that clear independently which for any characteristics of traders and assets is as efficient as a single exchange that clears all assets jointly (Section 4). Thus, a suitable design can implement a lower bound on welfare with no knowledge of traders' preferences or endowments. In fact, multi-venue design can be strictly more efficient than joint clearing (Section 5). Underlying these results is that innovation that would be neutral for traders' payoffs with joint clearing (if well-defined at all) is no longer redundant-another consequence of the nonproportionality between the price impact matrix $\boldsymbol{\Lambda}^{i}$ and the covariance matrix $\mathbf{\Sigma}$.

\subsubsection{Comparative Statics of Price Impact}

As anticipated by Example 1, Theorem 1, and Corollary 1, the price impact will be the key to understanding the design and welfare implications of independence in market clearing in Sections 4 and 5. Thus far we have noted that, in contrast to when markets clear jointly, the cross-exchange price impacts are zero (by definition of uncontingent demands) and the within-exchange price impacts change due to cross-asset inference (Theorem 1). One expects that these inference effects depend on the complementarities and substitutabilities in asset payoffs, which can be essentially arbitrary (subject to $\Sigma$ being a covariance matrix). Theorem 3 provides a sufficient condition for independent market clearing to increase the within-exchange price impacts $\left\{\lambda_{k}\right\}_{k}$.

Price impact and cross-asset inference. Consider the counterfactual that defines trader $i$ 's price impact in exchange $k$ : what is the effect of increasing the demand by trader $i$ for asset $k$ at a margin? Price $p_{k}$ increases so that other traders are willing to sell the extra units and the market clears. This direct effect is present in the contingent market as well. When the market is uncontingent, the change in price $p_{k}$ has also an indirect inference effect. Implicitly differentiating the first-order condition (10) of trader $j \neq i$ for asset $k$ with respect to $p_{k}$ characterizes the direct and inference effects on the marginal utility and the marginal payment:

$$
-\alpha^{j} \sigma_{k k} \frac{\partial q_{k}^{j}(\cdot)}{\partial p_{k}}-\underbrace{\sum_{\ell \neq k} \alpha^{j} \sigma_{k \ell} \frac{\partial q_{\ell}^{j}(\cdot)}{\partial p_{\ell}} \frac{\partial E\left[p_{\ell} \mid p_{k}, \mathbf{q}_{0}^{j}\right]}{\partial p_{k}}}_{\begin{array}{c}
\text { Inference effect } \\
\text { on the marginal utility }
\end{array}}=\underbrace{1}_{\begin{array}{c}
\text { Direct effect } \\
\text { on the marginal payment }
\end{array}}+\lambda_{k}^{j} \frac{\partial q_{k}^{j}(\cdot)}{\partial p_{k}} .
$$

Rewriting Eq. (19) decomposes the demand slope $\frac{\partial q_{k}^{j}(\cdot)}{\partial p_{k}}$ into the direct and indirect inference effects. Using $\frac{\partial q_{k}^{j}}{\partial p_{\ell}} \equiv \frac{\alpha^{j} \sigma_{k \ell}}{\alpha^{j} \sigma_{k k}+\lambda_{k}^{j}}\left(-\frac{\partial q_{\ell}^{j}(\cdot)}{\partial p_{\ell}}\right)=\frac{\alpha^{j} \sigma_{k \ell}}{\alpha^{j} \sigma_{k k}+\lambda_{k}^{j}} c_{\ell}^{j}$, we have

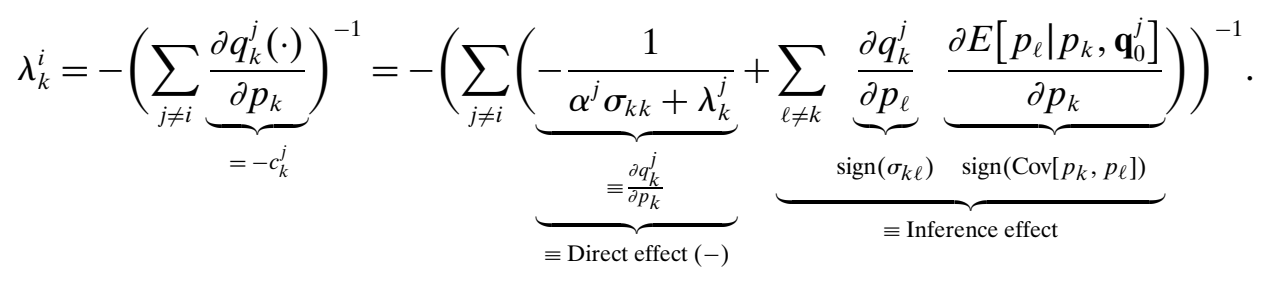

To explain the inference effect in Eq. (20), in the counterfactual following the demand increase by trader $i$, consider traders $j \neq i$ posterior conditioned on the higher price $p_{k}$. When asset payoffs are symmetric substitutes (i.e., $\sigma_{k k}=\sigma$ for all $k$ and $\sigma_{k \ell}=\sigma \rho>0$ 
for all $k$ and $\ell \neq k$ ), then other traders, who assume that all others-including trader $i$-play equilibrium, would instead attribute the higher price $p_{k}$ to a lower on average realization of endowments for all correlated assets, and expect higher prices and lower trades of those assets. ${ }^{29}$ This further increases the price at which they are willing to sell units of the substitute asset $k$ to trader $i .^{30}$

Theorem 3 shows that when payoff correlations are symmetric, uncontingent trading increases the within-exchange price impact-the inference effect in Eq. (20) is positive. Let $\rho_{k \ell} \equiv \operatorname{Corr}\left[r_{k}, r_{\ell}\right]=\frac{\sigma_{k \ell}}{\sqrt{\sigma_{k k} \sigma_{\ell \ell}}}$.

THEOREM 3-Price Impact: Comparative Statics: Suppose that asset covariances are symmetric: $\sigma_{\ell \ell}=\sigma$ for all $\ell$ and $\sigma_{\ell m}=\sigma \rho$ for all $\ell$ and $m \neq \ell$. The within-exchange price impact $\lambda_{k}$ satisfies the following properties for each $k$ :

(1) (Magnitude) With $K$ assets, price impact $\lambda_{k}$ maximally increases $K$-fold relative to $\lambda_{k}^{c}=\frac{\alpha}{I-2} \sigma_{k k}$ :

$$
\frac{\alpha}{I-2} \sigma_{k k} \leq \lambda_{k} \leq \frac{\alpha}{I-2} \sum_{\ell} \sigma_{\ell \ell}
$$

The upper bound of the $K$-fold increase is attained if and only if $|\rho|=1$.

(2) (Comparative statics) Relative to the contingent market:

(i) $\frac{\partial\left(\lambda_{k}-\lambda_{k}^{c}\right)}{\partial I}<0$, that is, the inference effect is decreasing in the number of traders I;

(ii) $\frac{\partial\left(\lambda_{k}-\lambda_{k}^{c}\right)}{\partial|\rho|}>0$, that is, the inference effect is increasing in asset correlation $|\rho|$.

As a corollary, in two-asset markets, uncontingent trading always increases the withinexchange price impact. ${ }^{31}$ Price impact $\lambda_{k}$ increases less relative to $\lambda_{k}^{c}=\frac{\alpha}{I-2} \sigma_{k k}$ when the inference effect is weaker-that is, with a larger number of traders $I$ or smaller correla-

\footnotetext{
${ }^{29}$ Price $p_{k}$ contains information about endowments of other traders for all assets and thus affects the conditional expectation separately from endowments $\mathbf{q}_{0}^{j}$ in expected trades $E\left[q_{\ell}^{j} \mid p_{k}, \mathbf{q}_{0}^{j}\right]$ (equivalently, $E\left[p_{\ell} \mid p_{k}, \mathbf{q}_{0}^{j}\right]$ ) provided that asset payoffs are not independent.

${ }^{30}$ The decomposition of the equilibrium price impact-which, by definition, represents an off-equilibrium counterfactual-captures how the cross-agent and cross-asset externalities are accounted for. This makes precise the difference with Nash-in-Nash (see Introduction).
}

${ }^{31}$ When $K=2$, Appendix C.2 characterizes $\frac{\partial E\left[p_{\ell} \mid p_{k}, \mathbf{q}_{0}^{j}\right]}{\partial p_{k}}$ as a closed-form function of the price impact $\lambda$ and simplifies Eq. (20) into Eq. (S92):

$$
\lambda_{k}=\underbrace{\frac{\alpha}{I-2}}_{=\lambda_{k}^{c}}+\underbrace{\frac{\alpha \rho}{I-2} \underbrace{\frac{2 x y}{x^{2}+y^{2}}}_{\operatorname{sign}(\rho)},}_{\text {Inference }(+)}
$$

where $x \equiv\left(1-\sigma_{0}\right)\left(1-\rho^{2}\right) \alpha+\left(1+(I-2) \sigma_{0}\right) \lambda$ and $y \equiv \rho\left(1+(I-2) \sigma_{0}\right) \lambda$. 


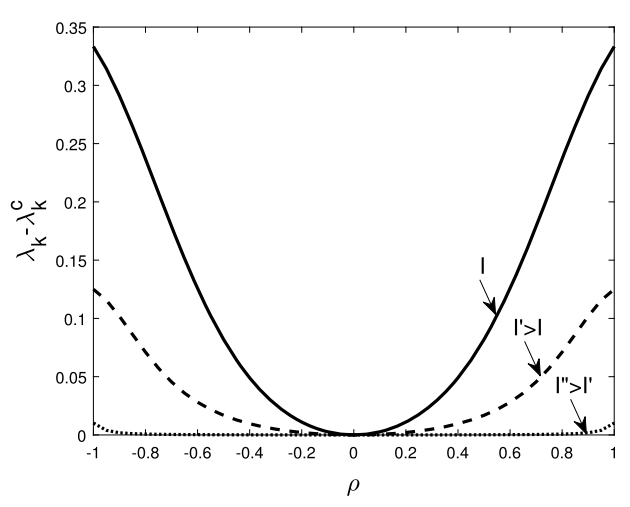

(A)

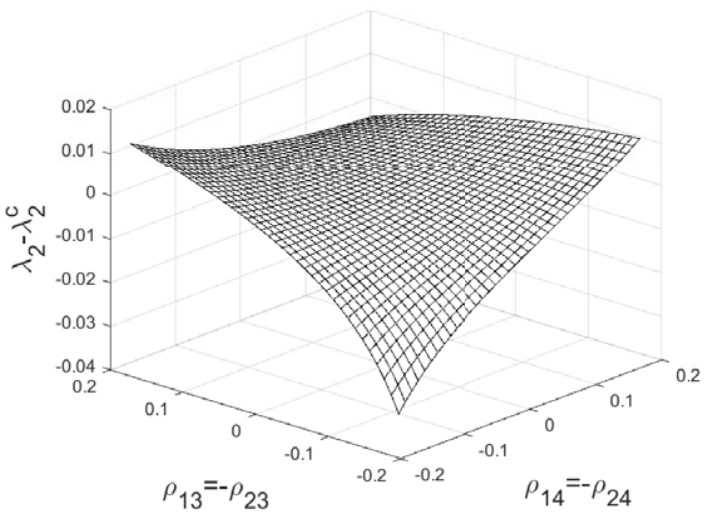

(B)

FIGURE 1.-Within-exchange price impact: inference effect. Notes: Panel A: $(K=2)$ Price impact difference $\lambda_{k}-\lambda_{k}^{c}$ is determined by the inference effect (Eq. (20)) - the direct effect is the same in contingent and uncontingent markets. The inference effect is larger (in absolute value) when assets are more strongly correlated (i.e., $|\rho|$ is larger) and the number of traders $I$ is smaller. The solid, dashed, and dotted curves assume, respectively, $I=5, I^{\prime}=10$, and $I^{\prime \prime}=100$. Panel B: $(K>2)$ With heterogeneous correlations, the price impact $\lambda_{k}$ can be lower than $\lambda_{k}^{c}$. Assets 1 and 2 are heterogeneously correlated with other assets: $\rho_{13}=\rho_{14}, \rho_{23}=\rho_{24}$, $\rho_{12}=-0.1, \rho_{15}=\rho_{16}=-0.5$, and $\rho_{25}=\rho_{26}=0.3 ; I=10$. In both panels, $\sigma_{c v}=0, \sigma_{p v}=1, \alpha=1$.

tions $|\rho|($ Figure $1(\mathrm{~A})) .{ }^{32}$ As $I \rightarrow \infty$, then $\Lambda^{i} \rightarrow 0$ for all $i\left(\right.$ Lemma S3 in Appendix B). ${ }^{33}$

When asset correlations are heterogeneous $(K>2)$, uncontingent trading can lower the price impact $\lambda_{k}$ for some assets relative to contingent trading (Figure 1(B)). In the counterfactual below Eq. (20), when correlations are symmetric, the inferred price changes of all assets induced by a demand change for one asset have the same sign. With heterogeneous correlations, however, the inferred price changes may differ in sign across assets, resulting in a negative "net" inference effect for asset $k$. This is because in traders' $j \neq i$ inference following trader $i$ 's demand increase, the expectation of a higher average realization of endowments for correlated assets-and hence lower prices and higher trades of those assets-dominates. This decreases the price at which they are willing to sell units of asset $k$ to trader $i$.

This result can also be seen in Corollary $1: \lambda_{k \ell}$ and $\operatorname{Cov}\left[p_{k}, p_{\ell}\right]$ depend on the covariance of all assets and need not match the sign of asset correlation (i.e., $\sigma_{k \ell}$ ); for example, the prices of complementary assets $\left(\sigma_{k \ell}<0\right)$ can be positively correlated $\left(\operatorname{Cov}\left[p_{k}, p_{\ell}\right]>\right.$ 0 ). As a result, $\lambda_{k}<\lambda_{k}^{c}$ is possible (see Eq. (20) and Figure 1(B)). (We provide additional discussion below the proof of Corollary S1 in Appendix B.)

\footnotetext{
${ }^{32}$ As discussed in Example 1, the welfare effects of independence in market clearing depend on cross-asset and within-exchange price impacts. The welfare effects of changes in $\left\{\lambda_{k}\right\}_{k}$ (which increase $K$-fold when assets are perfectly correlated) and $\left\{\lambda_{k \ell}\right\}_{\ell \neq k}$ (which equal zero) cancel out if and only if all assets are perfectly correlated (Corollary S2 in Appendix B). In symmetric-correlation markets of Theorem 3, Figure 1(A) shows that price impact $\lambda_{k}$ is convex in asset correlation $|\rho|$.

${ }^{33}$ The conditions from ft. 22 apply to contingent, uncontingent, and general markets in the next section. When schedules are not contingent, the cross-asset inference is present in the limit (i.e., $\frac{\partial E\left[p_{\ell} \mid p_{k}, \mathbf{q}_{0}^{j}\right]}{\partial p_{k}}=$ $\frac{\operatorname{Cov}\left[p_{\ell}, p_{k} \mid \mathbf{q}_{0}^{j}\right]}{\operatorname{Var}\left[p_{k} \mid \mathbf{q}_{0}^{j}\right]} \neq 0$ for $\ell \neq k$ ) even when the price impact becomes zero (i.e., $\lambda_{k}^{i} \rightarrow 0$ ).
} 


\section{CHANGES IN MARKET STRUCTURE}

The endogenous—-with limited conditioning-price covariance creates incentives for innovation in trading technology, defined by changes in market clearing. We first discuss a particular example of such innovation.

EXAMPLE 2-Innovation in Trading Technology: Suppose a new exchange for one of the $K$ traded assets is created to operate alongside the existing exchanges without altering traders' endowments of any asset. In the contingent market, the corresponding innovation of duplicating a traded asset would be neutral for traders' equilibrium payoffs. This can be seen from the first-order condition (4) for contingent demands $\mathbf{q}^{i, c}\left(p_{1}, \ldots, p_{K}, p_{K+1}\right)$ : $\mathbb{R}^{K+1} \rightarrow \mathbb{R}^{K+1}$,

$$
\left(\alpha^{i} \mathbf{\Sigma}^{+}+\Lambda^{i, c}\right) \mathbf{q}^{i, c}=\boldsymbol{\delta}^{+}-\mathbf{p}-\alpha^{i} \boldsymbol{\Sigma}^{+} \mathbf{q}_{0}^{i,+} \quad \forall \mathbf{p} \in \mathbb{R}^{K+1},
$$

where the payoffs of $K+1$ assets are jointly Normally distributed according to $\mathcal{N}\left(\boldsymbol{\delta}^{+}, \mathbf{\Sigma}^{+}\right)$ with $\boldsymbol{\delta}^{+} \in \mathbb{R}^{K+1}$ and $\boldsymbol{\Sigma}^{+} \in \mathbb{R}^{(K+1) \times(K+1)}$, and endowments for the duplicated asset can be arbitrarily split, provided that $q_{0, k}^{i,+}+q_{0, K+1}^{i,+}=q_{0, k}^{i}$. Using the fact that the price impact $\boldsymbol{\Lambda}^{i, c}=\beta^{i, c} \alpha^{i} \mathbf{\Sigma}^{+} \in \mathbb{R}^{(K+1) \times(K+1)}$ is proportional to the covariance matrix for all $i$ in the contingent market (Eq. (8)) and that the covariance matrix $\mathbf{\Sigma}^{+}$is singular with the new asset, condition (21) has a continuum of solutions $\mathbf{q}^{i, c} \in \mathbb{R}^{K+1}$ pointwise with respect to the price vector $\mathbf{p} \in \mathbb{R}^{K+1}$ including zero trade of the new asset $q_{K+1}^{i, c}(\cdot)=0$. Even if the asset in the new venue is traded, traders' equilibrium payoffs are the same as in the market with $K$ assets.

With independent market clearing, innovation in trading technology that would be neutral for traders' payoffs with joint clearing is generally no longer redundant, that is, traders' equilibrium payoffs change. In regard to innovation, we present two results: first, we characterize when innovation is not redundant (Proposition 4, Theorem 4); second, we show that markets with multiple exchanges that clear independently can be designed to function like a single exchange for all assets (Corollary 2). In Section 5, we examine how innovation affects welfare.

To accommodate various forms of innovation and a more general class of market structures, we extend the uncontingent model from Section 3.2. We allow arbitrary restrictions on cross-asset demand conditioning "between" uncontingent and contingent-this permits multiple assets per exchange-and we allow an asset to be traded in multiple venues. Given that all traders participate in all exchanges, we can identify an exchange with a subset of assets traded.

DEFINITION 4-Exchanges, Market Structure: Consider a market with $I$ traders and $K$ assets. An exchange $n$ is defined by the subset of assets traded $K(n) \subseteq K$. The market structure is described by a set of $N$ exchanges; that is, $N=\{K(n)\}_{n}$.

Exchanges clear independently: in each exchange $n$, trader $i$ submits a demand schedule $q_{k, n}^{i}(\cdot): \mathbb{R}^{K(n)} \rightarrow \mathbb{R}$ for each asset $k \in K(n)$ contingent on the prices of assets traded there, $\mathbf{p}_{K(n)} \equiv\left(p_{\ell, n}\right)_{\ell \in K(n)} \in \mathbb{R}^{K(n)}$. The market-clearing price vector $\mathbf{p}_{K(n)}$ in exchange $n$ is determined by $\sum_{j} q_{k, n}^{j}\left(\mathbf{p}_{K(n)}\right)=0$ jointly for all assets $k \in K(n)$ traded in this exchange.

Like in previous sections, the market clears independently across exchanges (but not necessarily across assets). The uncontingent market corresponds to $K$ exchanges $N=$ $\{\{k\}\}_{k}$, and the contingent market corresponds to a single exchange $N=\{K\}$. 
We treat the same asset traded in different exchanges as distinct assets with perfectly correlated payoffs. For the fundamentals $\boldsymbol{\delta} \in \mathbb{R}^{K}, \boldsymbol{\Sigma} \in \mathbb{R}^{K \times K}$, and $\left\{\mathbf{q}_{0}^{i} \in \mathbb{R}^{K}\right\}_{i}$, the superscript ' + ' indicates their counterparts in $\mathbb{R}^{\sum_{n} K(n)}$. Accordingly, the asset payoffs in $N$ exchanges are jointly Normally distributed $\mathcal{N}\left(\boldsymbol{\delta}^{+}, \boldsymbol{\Sigma}^{+}\right)$, where $\boldsymbol{\delta}^{+} \in \mathbb{R}^{\sum_{n} K(n)}$ and $\boldsymbol{\Sigma}^{+} \in \mathbb{R}^{\left(\sum_{n} K(n)\right) \times\left(\sum_{n} K(n)\right)}$. An asset's endowment can be split arbitrarily across exchanges. ${ }^{34}$ This is because the trader's demand for each asset depends on his total endowment of all assets (Eq. (22)), and hence so do prices. Generalizing from the first-order condition (11) for one asset per exchange, the best-response demand schedule for asset $k \in K(n)$ in exchange $n$ is determined by

$$
\delta_{k}^{+}-\alpha^{i} \boldsymbol{\Sigma}_{k} \mathbf{q}_{0}^{i}-\alpha^{i} \boldsymbol{\Sigma}_{k}^{+} E\left[\mathbf{q}^{i} \mid \mathbf{p}_{K(n)}, \mathbf{q}_{0}^{i}\right]=p_{k, n}+\left(\Lambda_{K(n)}^{i}\right)_{k} \mathbf{q}_{K(n)}^{i} \quad \forall \mathbf{p}_{K(n)} \in \mathbb{R}^{K(n)},
$$

given $\left\{q_{\ell, n}^{i}(\cdot)\right\}_{\ell \neq k, \ell \in K(n)}, \quad\left\{\left\{q_{\ell, n^{\prime}}^{i}(\cdot)\right\}_{\ell \in K\left(n^{\prime}\right)}\right\}_{n^{\prime} \neq n}, \quad F\left(\mathbf{s}^{-i} \mid \mathbf{q}_{0}^{i}\right)$, and $\left\{\boldsymbol{\Lambda}_{K(n)}^{i}\right\}_{n}$, where $\boldsymbol{\Lambda}_{K(n)}^{i} \in$ $\mathbb{R}^{K(n) \times K(n)}$ is trader $i$ 's price impact in exchange $n$ and $\left(\boldsymbol{\Lambda}_{K(n)}^{i}\right)_{k} \in \mathbb{R}^{1 \times K(n)}$ is the $k$ th row of $\boldsymbol{\Lambda}_{K(n)}^{i}$.

To analyze the equilibrium in markets with arbitrary demand conditioning across assets (Definition 4), we must extend Theorem 1. As with the simpler market structures characterized in Theorem 1, the fixed point in demand schedules is equivalent to a fixed point in traders' price impacts—now, block-diagonal matrices $\boldsymbol{\Lambda}^{i} \equiv \operatorname{diag}\left(\boldsymbol{\Lambda}_{K(n)}^{i}\right)_{n} \in$ $\mathbb{R}^{\left(\sum_{n} K(n)\right) \times\left(\sum_{n} K(n)\right)}$ for all $i$. Theorem 5 in Appendix A characterizes the equilibrium; Proposition S2 in Appendix C.1 provides the comparative statics of equilibrium price impact. The proofs of Proposition 2 and Theorem 2 in Appendix B encompass general market structures.

\subsection{Nonredundant Changes in Market Structure}

Price impacts per se are not useful in comparing payoffs across arbitrary market structures, as they are defined for different exchanges and may have different dimensionality. Proposition 4 in Appendix A simplifies and illuminates the analysis of nonredundancy and welfare, relating the payoffs across market structures with different conditioning variables, and hence a different price impact $\Lambda$, through a single-exchange counterfactual.

We define two statistics, $\widehat{\Lambda} \in \mathbb{R}^{K \times K}$ and $\widehat{\mathbf{B}} \in \mathbb{R}^{K \times K}$, that match the moments of total equilibrium trade of each asset $k$ across exchanges in a market structure $\{K(n)\}_{n}$, $\widehat{q}_{k}^{i} \equiv \sum_{\{n \mid k \in K(n)\}} q_{k, n}^{i}$. The per-unit price impact $\widehat{\Lambda}$ corresponds to the unique positive semidefinite matrix, such that if the price impact in a market structure with a single exchange for $K$ assets were $\widehat{\Lambda}$, the expected trade of each asset $k \in K$ in the counterfactual exchange would equal the expected equilibrium total trade in the market structure $\{K(n)\}_{n}$. For all $i$ and $k$,

$$
E\left[\widehat{q}_{k}^{i}\right] \equiv \sum_{\{n \mid k \in K(n)\}} E\left[q_{k, n}^{i}\right]=(\alpha \mathbf{\Sigma}+\widehat{\boldsymbol{\Lambda}})_{k}^{-1} \alpha \mathbf{\Sigma}\left(E\left[\overline{\mathbf{q}}_{0}\right]-E\left[\mathbf{q}_{0}^{i}\right]\right) .
$$

In turn, the cross-asset inference $\widehat{\mathbf{B}}$ is the coefficient on the privately known endowment $\mathbf{q}_{0}^{i}$ in a trader's total demand that matches the variance of the equilibrium total trade (cf.

\footnotetext{
${ }^{34}$ Given trader $i$ 's endowment $\mathbf{q}_{0}^{i}=\left(q_{0, k}^{i}\right)_{k} \in \mathbb{R}^{K}$, his endowment in $\mathbb{R}^{\sum_{n} K(n)}$ can be an arbitrary vector $\mathbf{q}_{0}^{i,+} \equiv$ $\left(\left(q_{0, k, n}^{i,+}\right)_{k}\right)_{n} \in \mathbb{R}^{\sum_{n} K(n)}$ such that $q_{0, k}^{i}=\sum_{\{n \mid k \in K(n)\}} q_{0, k, n}^{i,+}$ for all $k$. The parts of the split endowment in different exchanges are perfectly correlated: $\operatorname{Corr}\left[q_{0, k, n}^{i,+}, q_{0, k, n^{\prime}}^{i,+}\right]=1$ for any $n, n^{\prime}$ such that $k \in K(n) \cap K\left(n^{\prime}\right)$.
} 
Eq. (14)). For all $i$ and $k$,

$$
\operatorname{Var}\left[\widehat{q}_{k}^{i}\right] \equiv \operatorname{Var}\left[\sum_{\{n \mid k \in K(n)\}} q_{k, n}^{i}\right]=\widehat{\mathbf{B}} \operatorname{Var}\left[\overline{\mathbf{q}}_{0}-\mathbf{q}_{0}^{i}\right] \widehat{\mathbf{B}}^{\prime}=\frac{I-1}{I} \sigma_{p v} \widehat{\mathbf{B}}^{\prime}
$$

$\left(\widehat{\mathbf{B B}}^{\prime}\right)_{k \ell}\left(\widehat{\mathbf{B}}^{\prime}\right)_{k k}^{-1}$ is the cross-asset inference coefficient in the expected total trade $E\left[\widehat{q}_{\ell}^{i} \mid \widehat{q}_{k}^{i}\right.$, $\mathbf{q}_{0}^{i}$ ]. Proposition 4 (Appendix A) shows that one can compare equilibrium payoffs across market structures through $(\widehat{\boldsymbol{\Lambda}}, \widehat{\mathbf{B}}) \in \mathbb{R}^{K \times K} \times \mathbb{R}^{K \times K} .{ }^{35}$ Moreover, one can identify nonredundant innovation with the change in either statistic.

Which innovations are nonredundant? Not all are. Theorem 4 shows that the asymmetry of price impact is the key to understanding which innovations are nonredundant. ${ }^{36}$

THEOREM 4-Nonredundancy of Changes in Market Structure: Conditions: Let I $<$ and $K>1$, and consider a market structure $N=\{K(n)\}_{n}$. Suppose a new exchange $n^{\prime}$ such that $K\left(n^{\prime}\right) \subset K(n)$ for some $n \in N$ is introduced. Exchange $n^{\prime}$ is redundant in an equilibrium if and only if one of the following conditions holds:

(i) (Innovation mimics an exchange) The set of assets traded in exchange $n^{\prime}$ is the same as in an existing exchange, that is, $K\left(n^{\prime}\right)=K\left(n^{\prime \prime}\right)$ for some $n^{\prime \prime} \in N$.

(ii) (Symmetric price impact) The price impact in an exchange $n^{\prime \prime}$ such that $K\left(n^{\prime}\right) \subset$ $K\left(n^{\prime \prime}\right)$ is symmetric, that is, $\boldsymbol{\Lambda}_{K\left(n^{\prime \prime}\right)}=\Lambda_{K\left(n^{\prime \prime}\right)}^{\prime}$.

(iii) (Independent or perfectly correlated assets) The payoffs of all assets in $K\left(n^{\prime}\right)$ are independent or perfectly correlated with those of the assets in $K(n) \backslash K\left(n^{\prime}\right)$, that is, $\left|\rho_{k \ell}\right| \in\{0,1\}$ for all $k \in K\left(n^{\prime}\right)$ and $\ell \in K(n) \backslash K\left(n^{\prime}\right)$.

So far, we have emphasized that independent market clearing severs the (proportional) relation between the price impact, and hence cross-asset demand substitution (i.e., the inverse of Jacobian $\mathbf{C}$ ), and asset covariance. The asymmetry of the equilibrium price impact matrix is a new equilibrium property relative to both the contingent design $\left(\Lambda^{c}\right.$ is proportional to $\Sigma$ and hence $\lambda_{k \ell}=\lambda_{\ell k}, \ell \neq k$, Eq. (8)) and the uncontingent design $\left(\lambda_{k \ell}=0, \ell \neq k\right)$. In more general designs, the price impact is symmetric only under a joint symmetry restriction on the covariance $\Sigma$ and the market structure $N=\{K(n)\}_{n}$. The cross-asset price impacts are asymmetric because the cross-asset inference effects are (Section 3.2.3). Example 3 illustrates this link.

By Proposition 4, an innovation is nonredundant if it changes the relative trading costs across assets $\widehat{\boldsymbol{\Lambda}}$ or cross-asset inference $\widehat{\mathbf{B}}$. Both $\widehat{\boldsymbol{\Lambda}}$ and $\widehat{\mathbf{B}}$ change if one does, generically in asset covariance $\boldsymbol{\Sigma}$ and market structure $N=\{K(n)\}_{n}$ (by the proof of Theorem 4). Intuitively, under any of Theorem 4's conditions, in any market structure, the prices of the same asset traded in different venues equalize (Lemma S4 in Appendix B); new exchanges do not create additional linearly independent conditioning variables in any asset's demand. Neither $\widehat{\mathbf{B}}$ nor $\widehat{\boldsymbol{\Lambda}}$ change. However, when the price impact is asymmetric, the

\footnotetext{
${ }^{35} \widehat{\boldsymbol{\Lambda}}$ and $\widehat{\mathbf{B}}$ are not defined as equilibrium objects in a single-exchange game. While either price impact $\boldsymbol{\Lambda}$ itself or the demand coefficient $\mathbf{B}$ is a sufficient statistic for equilibrium payoffs in a market structure $N=$ $\{K(n)\}_{k}$ (Theorems 1 and 5), their per-unit counterparts $\widehat{\boldsymbol{\Lambda}}$ and $\widehat{\mathbf{B}}$ are both required for the payoffs in two market structures $N$ and $N^{\prime}$ to match, unless price impact is a symmetric matrix.

${ }^{36}$ Mathematically, the relevance of the price impact asymmetry can be seen in Eq. (49): the inverse matrix in $\widehat{\mathbf{B}}$ is a harmonic mean of the demand coefficient $\mathbf{C}=\frac{1}{I-1}\left(\boldsymbol{\Lambda}^{-1}\right)^{\prime}$ and its transpose $\mathbf{C}^{\prime}$ and, thus, it is not a linear function of $\widehat{\Lambda}$ unless $\boldsymbol{\Lambda}$ is symmetric.
} 
prices of the same asset traded in different venues differ, and thus both $\widehat{\mathbf{B}}$ and $\widehat{\boldsymbol{\Lambda}}$ change. Example 3 illustrates Theorem 4.

EXAMPLE 3-Nonredundant Exchanges and Price Impact Asymmetry: Consider a market with two exchanges $N=\{\{1,2\},\{3\}\}$. For simplicity, assume that $\sigma_{11}=\sigma_{22}=\sigma_{33}$ and the assets are imperfectly correlated $\left(0<\left|\rho_{k \ell}\right|<1\right.$ for all $k$ and $\left.\ell \neq k\right)$. Per Theorem 4(ii), the introduction of exchange $\{1\}$ is redundant if and only if the equilibrium price impact $\Lambda_{\{1,2\}}$ is a symmetric matrix, that is, the cross-asset price impacts coincide, $\lambda_{12}=\lambda_{21}$.

(a) When is price impact $\boldsymbol{\Lambda}_{\{1,2\}}$ symmetric? This is the case if and only if the covariances of assets 1 and 2 are symmetric:

$$
\sigma_{13}=\sigma_{23} \Leftrightarrow \operatorname{Cov}\left[p_{2}, p_{3}\right]=\operatorname{Cov}\left[p_{1}, p_{3}\right] .
$$

A closer look at the inference effect in price impact shows why. Using the relation between the price impact and demand slope, $\boldsymbol{\Lambda}_{\{1,2\}}=\frac{1}{I-1}\left(\mathbf{C}_{\{1,2\}}^{-1}\right)^{\prime}$, that is,

$$
\left[\begin{array}{ll}
\lambda_{11} & \lambda_{12} \\
\lambda_{21} & \lambda_{22}
\end{array}\right]=\frac{1}{I-1} \frac{1}{c_{11} c_{22}-c_{12} c_{21}}\left[\begin{array}{cc}
c_{22} & -c_{21} \\
-c_{12} & c_{11}
\end{array}\right] \text {, }
$$

we decompose the off-diagonal demand coefficients $c_{12} \equiv \frac{\partial q_{1}^{j}(\cdot)}{\partial p_{2}}$ and $c_{21} \equiv \frac{\partial q_{2}^{j}(\cdot)}{\partial p_{1}}$ into direct and indirect effects (analogously to Eq. (20) in Section 3.2.3):

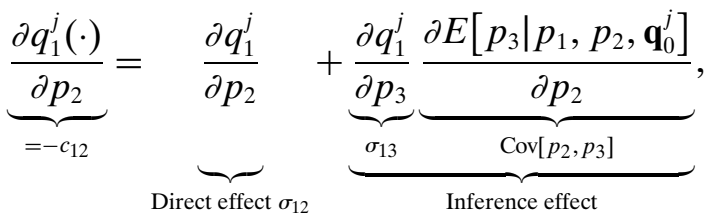

$$
\begin{aligned}
& \underbrace{\frac{\partial q_{2}^{j}(\cdot)}{\partial p_{1}}}_{=-c_{21}}=\underbrace{\frac{\partial q_{2}^{j}}{\partial p_{1}}}_{\text {Direct effect } \sigma_{21}}+\underbrace{\frac{\partial q_{2}^{j}}{\partial p_{3}} \underbrace{\frac{\partial E\left[p_{3} \mid p_{1}, p_{2}, \mathbf{q}_{0}^{j}\right]}{\partial p_{1}}}_{\sigma_{23}}}_{\text {Inference effect }} .
\end{aligned}
$$

Since the direct effects in cross-asset price impact coincide, the price impact is symmetric if the corresponding inference effects coincide. As we discussed in Section 3.2.3, because the price impact is not proportional to covariance $\mathbf{\Sigma}$ with independent market clearing, the price impact between any pair of assets depends on the covariance of all assets. Suppose that assets 1 and 2, which clear jointly and hence involve no mutual inference effects, are asymmetrically correlated with asset 3 . For instance, let asset 2 be uncorrelated with asset 3 . Then, a trader's demand increase for asset 2 as $p_{1}$ changes has no inference effect on the price of asset 3 , but a demand increase for asset 1 as $p_{2}$ changes has a nonzero inference effect.

(b) Why is exchange $\{1\}$ not redundant when the cross-asset price impacts $\lambda_{12}$ and $\lambda_{21}$ are asymmetric (Theorem 4(ii))? Intuitively, for the new exchange to be nonredundant, it must be that the equilibrium prices of asset 1 in exchanges $\{1,2\}$ and $\{1\}$ carry different information. Price impacts determine the weights in the price function on the random variables - the aggregate endowments $\left\{\bar{q}_{0, k}\right\}_{k}$ (see Eq. (17)). In particular, the matrix weight coefficient $\mathbf{C}^{-1} \mathbf{B}$ is determined by the price impact $\boldsymbol{\Lambda}$ and its transpose $\boldsymbol{\Lambda}^{\prime}$ (which 
is not relevant with contingent or uncontingent demands). With asymmetric price impact, the weights on the aggregate endowments in prices of the same assets in different exchanges are distinct. ${ }^{37}$

(c) On the other hand, the introduction of exchange $\{3\}$ is redundant irrespective of the symmetry of $\boldsymbol{\Lambda}$ (Theorem 4(i)). Because the inference effects with respect to assets 1 and 2 are the same in both exchanges $\{3\}$, the price impact is the same in these venues-prices equalize, and traders split their demands for asset 3 equally between the two exchanges.

We highlight additional insights of Proposition 4 and Theorem 4. These results underscore the role of imperfect competition for the nonredundancy of innovation. Given the market structure with multiple trading venues that clear independently, there is more nonredundant innovation when traders have price impact:

- Exchanges $n^{\prime}$ whose assets are not a subset of another venue's assets $\left(K\left(n^{\prime}\right) \not \subset K(n)\right.$ for all $n$ ): the total demand for some asset $k \in K$ is contingent on the prices of new assets; for example, exchange $\{2,3\}$ in Example 3 . The inference error weakly decreases for all assets.

- Exchanges $n^{\prime}$ whose assets are a proper subset of another venue's assets $\left(K\left(n^{\prime}\right) \subsetneq\right.$ $K(n)$ for some $n)$ : the total demand of each asset $k \in K$ is contingent on prices of the same assets; for example, exchange $\{1\}$ in Example 3. However, with new contingent variables (i.e., additional prices of the same assets), demands for the same asset in different exchanges are contingent on distinct linear combinations of the random variables. This changes cross-asset inference, and hence $\widehat{\boldsymbol{\Lambda}}$ and $\widehat{\mathbf{B}}$. Inference error can increase for some assets and decrease for others.

The latter type of nonredundant innovation, which is present when price impact is asymmetric (Theorem 4(ii)), has no counterparts in competitive markets. Indeed, when the price impact is symmetric (e.g., the zero matrix), only the former type of innovation can be nonredundant. Furthermore, even if inference error were zero (i.e., $\left(\sigma_{c v}, \sigma_{p v}\right) \rightarrow 0$, $\left.\frac{\sigma_{p v}}{\sigma_{c v}}>0\right)$, the former type of innovation would be nonredundant when $I<\infty$ but not when $\stackrel{\sigma_{c v}}{\rightarrow} \rightarrow \infty$, as it would change the equilibrium price impact.

More generally, apart from the introduction of new trading protocols (Example 3), independent market clearing motivates other forms of innovation, such as the linking of existing trading protocols (i.e., merging assets between venues), and the inclusion of an asset in a trading protocol where it was not previously traded (e.g., asset listings). When increasing the set of (imperfectly correlated) conditioning variables in traders' demands, these innovations lead to a market structure with more, fewer, and the same number of exchanges, respectively. ${ }^{38}$

\subsection{Multiple Exchanges: Equivalence With Joint Market Clearing}

In this section, we ask: what is the scope for innovation in trading technology that would not be redundant in the market? Corollary 2 characterizes the bound on the number of nonredundant exchanges in any market structure. The intuition for the general result can be gleaned from the following example.

\footnotetext{
${ }^{37}$ Per Theorem 4(ii), the symmetry of the price impact is required only for the exchanges $n^{\prime \prime}$ whose assets are a superset of those in the new venue $n^{\prime}$. For example, in the market $\{\{1,2\},\{3,4\}\}$, the price impact in exchange $\{3,4\}$ need not be symmetric for exchange $\{1\}$ to be redundant. The required symmetry condition ensures that the inference effects among the new assets $K\left(n^{\prime}\right)$ and between assets $K\left(n^{\prime}\right)$ and assets $K(n) \backslash K\left(n^{\prime}\right)$ and $K \backslash K(n)$ are symmetric.

${ }^{38}$ The argument from the proof of Theorem 4 applies to these other innovations: an innovation is redundant if the price impact submatrix that corresponds to the affected exchanges is symmetric.
} 
EXAMPLE 4-Multi-Venue Market Can Be Equivalent to Market That Clears Jointly: Consider the market structure $\{\{1,2\},\{2,3\},\{3,1\}$. Even though the market is composed of multiple exchanges, none of which contain all assets, traders' equilibrium payoffs are the same as in the market with a single exchange for all assets $\{\{1,2,3\}\}$.

To explain this result, we consider trader $i$ 's total demand for asset 1 behind the oneexchange counterfactual in Proposition 4, that is, the sum of his demands for asset 1 in exchanges $\{1,2\}$ and $\{3,1\}$ :

$$
\widehat{q}_{1}^{i}\left(\mathbf{p}_{\{1,2\}}, \mathbf{p}_{\{3,1\}}\right) \equiv q_{1,\{1,2\}}^{i}\left(\mathbf{p}_{\{1,2\}}\right)+q_{1,\{3,1\}}^{i}\left(\mathbf{p}_{\{3,1\}}\right) \quad \forall \mathbf{p}_{\{1,2\}} \in \mathbb{R}^{2} \forall \mathbf{p}_{\{3,1\}} \in \mathbb{R}^{2} .
$$

In either exchange, the expected trades are conditioned on the respective contingent variables in traders' demands-for example, $p_{1}$ and $p_{2}$ in exchange $\{1,2\}$. In the total demand for asset 1 , with additional expected trade terms contingent on a different subset of prices, the expected trades of assets 2 and 3 are linear combinations of all random variables, $\bar{q}_{0,1}, \bar{q}_{0,2}$, and $\bar{q}_{0,3}{ }^{39}$

Crucially, since the total demand for each asset is conditioned on at least $K=3$ prices that give linearly independent combinations of the $K$ random variables $\left\{\bar{q}_{0, k}\right\}_{k}$, the inference errors cancel out: the sum of expected total trades $E\left[\widehat{q}_{\ell}^{i} \mid \mathbf{p}_{K(n)}, \mathbf{q}_{0}^{i}\right]$ in the total demand for asset $k$ across exchanges $\{n \mid k \in K(n)\}$ is the same as $\widehat{q}_{\ell}^{i}$ for all $k$ and $\ell \neq k$. Equilibrium can be ex post even if traders' demand schedules do not condition on prices of all assets in any exchange (i.e., $K(n) \subsetneq K$ for all $n$ ) so that no expectation about trade is perfect, that is, $E\left[q_{\ell, n^{\prime}}^{i} \mid \mathbf{p}_{K(n)}, \mathbf{q}_{0}^{i}\right] \neq q_{\ell, n^{\prime}}^{i}$ for all $n$ and $n^{\prime} \neq n$, and all $i$. Then, with perfect inference in total demands, the price impact matrix is symmetric and the same as in the contingent market, $\widehat{\boldsymbol{\Lambda}}=\boldsymbol{\Lambda}^{c}=\frac{\alpha}{I-2} \boldsymbol{\Sigma}$ and $\widehat{\mathbf{B}}=\mathbf{B}^{c}=\frac{I-2}{I-1} \mathbf{I d}$ (Proposition 4). The cross-exchange inference effects mimic the contingent design's cross-asset price impact; equilibrium is as if traders could condition their demand for each asset on the price vector. ${ }^{40}$

Corollary 2 gives a condition on the market structure itself that characterizes the scope for nonredundant innovation. ${ }^{41}$

COROLlaRY 2-Redundancy of Changes in Market Structure: A Condition on Exchanges: Suppose that $0<\left|\rho_{k \ell}\right|<1$ for some $k$ and $\ell \neq k$. When $I<\infty$, the following statements are equivalent:

(i) Introducing any additional exchange $n^{\prime}$ is redundant;

(ii) Equilibrium is ex post;

(iii) For every pair of assets $k^{\prime}$ and $\ell^{\prime} \neq k^{\prime}$ such that $0<\left|\rho_{k^{\prime} \ell^{\prime}}\right|<1$, there is an exchange $n$ in which these assets are traded, that is, $k^{\prime}, \ell^{\prime} \in K(n)$.

The equivalence between conditions (ii) and (iii) answers the following question: Given the assets and traders, which market structures with multiple exchanges that clear independently function like a market that clears jointly, and when does equilibrium behavior

\footnotetext{
${ }^{39}$ For example, from Eq. (22), the total expected trade for asset 2 in total demand $\widehat{q}_{1}^{i}(\cdot)=q_{1,\{1,2\}}^{i}(\cdot)+q_{1,\{3,1\}}^{i}(\cdot)$ is $\left(\left(\alpha \boldsymbol{\Sigma}_{\{1,2\},\{1,2\}}+\boldsymbol{\Lambda}_{\{1,2\}}\right)^{-1}\right)_{1} \alpha \boldsymbol{\Sigma}_{\{1,2\}} E\left[\widehat{q}_{2}^{i} \mid \mathbf{p}_{\{1,2\}}, \mathbf{q}_{0}^{i}\right]+\left(\left(\alpha \boldsymbol{\Sigma}_{\{3,1\},\{3,1\}}+\boldsymbol{\Lambda}_{\{3,1\}}\right)^{-1}\right)_{1} \alpha \boldsymbol{\Sigma}_{\{3,1\}} E\left[\widehat{q}_{2}^{i} \mid \mathbf{p}_{\{3,1\}}, \mathbf{q}_{0}^{i}\right]$.

${ }^{40}$ Consider a market structure $\{\{1,2\},\{2,3\},\{3,1\},\{4\}\}$ and assume that the payoff of asset 4 is imperfectly correlated with those of other assets. This market structure is payoff-equivalent to $\{\{1,2,3\},\{4\}\}$ if and only if the inference effects of assets 1,2 . and 3 with respect to asset 4 are symmetric. Then, multiple venues that clear independently can implement joint clearing "locally" for assets 1,2, and 3.

${ }^{41}$ Corollary S2 in Appendix B shows that as long as some assets in the market are imperfectly correlated, some innovations will not be redundant. With perfectly correlated assets, inference is perfect; with independent assets, inference is not payoff-relevant. Innovation then does not affect equilibrium inference, and hence price impact.
} 
differ? This result shows that for all market structures characterized in condition (iii) of Corollary 2, one can implement the contingent-market outcome via schedules with simpler conditioning. ${ }^{42}$ Two assets per exchange suffice.

The equivalence between conditions (i) and (iii) puts a bound on the number of exchanges that can be introduced in a market-or the ways in which trading protocols can be linked (by merging their assets), or asset listings—and still be nonredundant. The maximal number of such nonredundant innovations is $\frac{K(K-1)}{2}$.

\section{WELFARE AND INDEPENDENCE IN MARKET CLEARING}

In this section, we consider the welfare impact of independence in market clearing. An important implication of Corollary 2 is that when combined with a suitable exchange design, markets with multiple exchanges that clear independently can be as efficient as a single exchange that clears all assets jointly, for any distributions of asset payoffs and endowments. The main observation in this section is that markets with multiple exchanges can strictly improve ex ante welfare relative to joint market clearing.

We first ask: Why would trading platforms not allow traders to condition their demands on more information from other venues or trading protocols within the platform? If the market were competitive $(I \rightarrow \infty)$, then joint clearing would give higher welfare than any other market structure-it would eliminate inference error across exchanges. In imperfectly competitive markets, the benefit of lower trading costs associated with price impact can counteract the cost that stems from inference error.

We then inquire: In which trading environments is welfare higher with multi-venue designs? Unlike the competitive market, efficient design depends on market characteristics. Corollary 3, Proposition 3, and Examples 1 and 5 give and illustrate the conditions.

\subsection{Welfare-Improving Designs}

The ex ante total welfare is given by the sum of the equilibrium payoffs for all traders:

$$
\begin{aligned}
E\left[u^{i}\left(\mathbf{q}^{i}\right)-\mathbf{p} \cdot \mathbf{q}^{i}\right]= & \underbrace{E\left[\boldsymbol{\delta} \cdot \mathbf{q}_{0}^{i}-\frac{1}{2} \mathbf{q}_{0}^{i} \cdot \alpha \boldsymbol{\Sigma} \mathbf{q}_{0}^{i}\right]}_{\text {Payoff without trade }}+\underbrace{\left(E\left[\overline{\mathbf{q}}_{0}\right]-E\left[\mathbf{q}_{0}^{i}\right]\right) \cdot \mathbf{Y}(\widehat{\boldsymbol{\Lambda}})\left(E\left[\overline{\mathbf{q}}_{0}\right]-E\left[\mathbf{q}_{0}^{i}\right]\right)}_{\text {Equilibrium surplus from trade }} \\
& +\underbrace{\left.\frac{1}{\frac{I-1}{I} \sigma_{p v} \operatorname{tr}\left(\widehat{\mathbf{B}}^{\prime} \alpha \boldsymbol{\Sigma}\right.}+\alpha \boldsymbol{\Sigma} \widehat{\mathbf{B}}-\widehat{\mathbf{B}}^{\prime} \alpha \mathbf{\Sigma} \widehat{\mathbf{B}}\right)}_{\text {Payoff term due to } \operatorname{Var}\left[\overline{\mathbf{q}}_{0} \mid \mathbf{q}_{0}^{i}\right]>0},
\end{aligned}
$$

where the per-unit price impact $\widehat{\Lambda} \in \mathbb{R}^{K \times K}$ and cross-asset inference $\widehat{\mathbf{B}} \in \mathbb{R}^{K \times K}$ are defined by conditions (23) and (24), respectively. When the market structure changes, the corresponding welfare change can be decomposed into three effects related to: (1) the price impact for a given asset (i.e., $\left.\widehat{\lambda}_{k}\right)$, (2) the cross-asset price impact (i.e., $\widehat{\lambda}_{k \ell}$ ), and (3) the inference error. ${ }^{43}$ A corollary of Theorem 3 and Proposition 4 shows that it is important to

\footnotetext{
${ }^{42}$ For "(iii) implies (ii)," we select the equilibrium with symmetric price impact (i.e., $\lambda_{k \ell}=\lambda_{\ell k}$ ) whenever it exists. We have not found any parameters with which an asymmetric equilibrium exists when condition (iii) holds.

${ }^{43}$ The inference error in the last term of $\sum_{i} E\left[u^{i}\left(\mathbf{q}^{i}\right)-\mathbf{p} \cdot \mathbf{q}^{i}\right]$ in Eq. (25) can be characterized as

$$
(I-1) \sigma_{p v} \operatorname{tr}\left(\frac{1}{2}\left(\widehat{\mathbf{B}}-\mathbf{B}^{c}\right)^{\prime} \alpha \mathbf{\Sigma}\left(\widehat{\mathbf{B}}-\mathbf{B}^{c}\right)+\frac{1}{I-1} \alpha \mathbf{\Sigma}\left(\widehat{\mathbf{B}}-\mathbf{B}^{c}\right)\right),
$$


distinguish between the trading cost components (1) and (2). Respectively, they represent the trading cost of risk sharing and the trading costs of diversification.

COROLlARY 3-Price Impact and Market Structure: Consider two market structures $N$ and $N^{\prime}$ such that if $\{k, \ell\} \subset K\left(n^{\prime}\right)$ for some $n^{\prime} \in N^{\prime}, \ell \neq k$, then $\{k, \ell\} \subset K(n)$ for some $n \in N$. Let $\widehat{\Lambda}^{N}$ and $\widehat{\Lambda}^{N^{\prime}}$ be the corresponding per-unit price impact matrices. Assume that $\widehat{\Lambda}^{N} \neq \widehat{\Lambda}^{N^{\prime}}: 44$

(i) If $K=2$, then $\hat{\boldsymbol{\lambda}}_{k}^{N} \leq \hat{\boldsymbol{\lambda}}_{k}^{N^{\prime}}$ for each $k$.

(ii) If $K>2, \hat{\boldsymbol{\lambda}}_{k}^{N}$ need not be lower than $\hat{\boldsymbol{\lambda}}_{k}^{N^{\prime}}$ for some $k$.

For two-asset markets, Corollary 3 establishes a trade-off between risk sharing and diversification. Namely, when $K=2$, then limited conditioning increases the per-unit diagonal price impact for each asset (part (i)). ${ }^{45}$ Although joint clearing minimizes the cost of risk sharing, multi-venue trading can strictly increase welfare by lowering the trading cost of diversification that stems from cross-asset price impact; Example 1 illustrates this. Corollary 4 in Section 5.2 provides the necessary and sufficient conditions for independent market clearing to increase welfare in the Pareto sense in two-asset markets.

More generally, in markets with $K>2$, multi-venue trading can increase welfare by lowering the trading costs of risk sharing, diversification, or both (part (ii); Example 5). Likewise, so can the further limiting of demand conditioning in markets with multiple exchanges. The increase in welfare with multi-venue trading-relative to joint clearing or more generally_can be accomplished in the Pareto sense.

\subsection{When Are Multi-Venue Markets More Efficient?}

We further ask: Which designs are more efficient? Proposition 3 translates Corollary 3's price-impact effects on risk sharing and diversification to market characteristics.

PROPOSITION 3-Welfare With Multiple Exchanges Versus Joint Market Clearing: Given $I<\infty$ traders and $K$ assets such that $0<\left|\rho_{k \ell}\right|<1$ for some $k$ and $\ell \neq k$, there exists a market structure with multiple exchanges for which the ex ante welfare is strictly larger than that in a single exchange for some distribution of endowments $\left\{\mathbf{q}_{0}^{i}\right\}_{i}$.

Proposition 3, Corollary 4, and Example 5 demonstrate that neither the contingent nor the uncontingent market structure is efficient regardless of the market characteristics (see also Example 1 and Corollary 3). Here, we highlight three insights.

First, if the number of traders is sufficiently large, then joint clearing (or an equivalent design) is efficient. Given any $K$ (imperfectly correlated) assets and $I<\infty$ traders, however, a market structure with multiple venues is more efficient than joint clearing for some distributions of endowments. The proof of Proposition 3 is constructive and provides a sufficient condition on the pertinent market structures: any demerger of a single exchange for all assets.

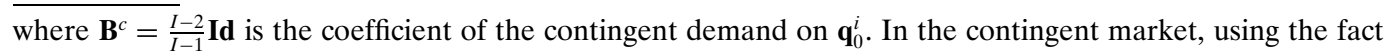
that $\widehat{\boldsymbol{\Lambda}}=\boldsymbol{\Lambda}^{c}$, we have that $\Upsilon\left(\boldsymbol{\Lambda}^{c}\right) \equiv \frac{I(I-2)}{2(I-1)^{2}} \alpha \boldsymbol{\Sigma}$ in the ex ante welfare Eq. (25), and the inference error (26) is zero (equilibrium is ex post).

${ }^{44}$ That is, there exists $\left\{k^{\prime}, \ell^{\prime}\right\}$ such that $\left\{k^{\prime}, \ell^{\prime}\right\} \subset K(n)$ for some $n \in N$ but $\left\{k^{\prime}, \ell^{\prime}\right\} \not \subset K\left(n^{\prime}\right)$ for all $n^{\prime} \in N^{\prime}$.

${ }^{45}$ Part (i) of Corollary 3 holds in more general markets with $K \geq 2$ assets, symmetric covariances (i.e., $\sigma_{k k}=\sigma$ for all $k$ and $\sigma_{k \ell}=\sigma \rho$ for all $k$ and $\ell \neq k$ ), and market structures defined by symmetric demergers (Definition 5). (See Proposition S2 in Appendix C.1.) 
DEFINITION 5-Demerger: A demerger of a single exchange for all assets is a market structure $N=\{K(n)\}_{n}$ whose exchanges partition the $K$ assets traded: $K(n) \cap K\left(n^{\prime}\right)=\emptyset$ for all $n$ and $n^{\prime} \neq n$. A demerger is symmetric if its exchanges have the same number of assets.

Thus, simply breaking up a single exchange for all assets into multiple venues can increase welfare.

A market with multiple trading venues yields higher welfare than a single exchange if $E\left[\overline{\mathbf{q}}_{0}\right]-E\left[\mathbf{q}_{0}^{i}\right]$ is proportional to an eigenvector of $\mathbf{Y}\left(\boldsymbol{\Lambda}^{c}\right)-\mathbf{Y}(\widehat{\Lambda})$ that corresponds to a negative eigenvalue (see Eq. (25)). Intuitively, in light of the trade-off between price impact and information loss implied by the multi-venue design relative to the contingent one, efficient market structure reduces the (net) cost of within- and across-exchange price impacts $\mathbf{Y}\left(\boldsymbol{\Lambda}^{c}\right)-\mathbf{Y}(\widehat{\boldsymbol{\Lambda}})$, given the trading needs $E\left[\overline{\mathbf{q}}_{0}\right]-E\left[\mathbf{q}_{0}^{i}\right]$. Corollary 3 and Lemma S5 in Appendix B give a sufficient condition on the market structures to admit the cost reduction: a negative eigenvalue exists for any market structure whose exchanges are demergers of a single venue for all assets (Definition 5). Proposition 3-like all results in the paperis robust by continuity (i.e., by the continuity of the price impact and the total welfare with respect to the endowment distribution).

Second, in which trading environments is multi-venue trading more efficient than joint clearing? Any change in design affects price impacts for all assets ${ }^{46}$ and these effects depend on the market structure and characteristics of traders and assets. The proof of Proposition 3 shows that the welfare impact of a change in design can be determined by a joint condition on the primitives: the joint substitution in asset payoffs (i.e., $\mathbf{\Sigma}$ ) and traders' endowments (i.e., $\left.\left\{\left|E\left[\bar{q}_{0, k}\right]-E\left[q_{0, k}^{i}\right]\right|\right\}_{i, k}\right) .{ }^{47}$ In particular, even if all asset payoffs are symmetric substitutes or complements, efficient design depends on whether some traders buy and others sell assets (e.g., the primary market in Treasury auctions) or traders buy some assets and sell others (e.g., inter-dealer markets). See Examples 1 and 5(a) and (b).

Finally, the heterogeneity in the joint substitution $\left\{\boldsymbol{\Sigma},\left\{E\left[\overline{\mathbf{q}}_{0}\right]-E\left[\mathbf{q}_{0}^{i}\right]\right\}_{i}\right\}$ determines whether the net benefit from diversification and risk sharing dominates with multivenue trading. With sufficient heterogeneity, inducing asymmetries in trading costs is beneficial-market structures "intermediate" between contingent or uncontingent are then efficient. See Example 5(c).

Corollary 4 illustrates these observations in two-asset markets, providing a necessary and sufficient condition on $\left\{\boldsymbol{\Sigma},\left\{E\left[\overline{\mathbf{q}}_{0}\right]-E\left[\mathbf{q}_{0}^{i}\right]\right\}_{i}\right\}$ for multi-venue trading to dominate joint clearing in welfare terms. ${ }^{48}$

\footnotetext{
${ }^{46}$ Another result of the non-proportionality of the price impact to asset covariance in markets other than the contingent one (Eqs. (16) and (S22)).

${ }^{47}$ The role of the joint condition can be seen in Eq. (25), where the equilibrium surplus is a quadratic matrix function of expected trading needs $\left\{E\left[\overline{\mathbf{q}}_{0}\right]-E\left[\mathbf{q}_{0}^{i}\right]\right\}_{i}$ and the surplus matrix difference $\mathbf{Y}\left(\boldsymbol{\Lambda}^{c}\right)-\mathbf{Y}(\widehat{\boldsymbol{\Lambda}})$ depends on the asset covariance $\boldsymbol{\Sigma}$. In any demerger (including uncontingent markets), the price impact matrices in the surplus matrix difference $\mathbf{Y}\left(\boldsymbol{\Lambda}^{c}\right)-\mathbf{Y}(\widehat{\Lambda})$ are not ranked in a positive semi-definite sense (Corollary 3 ). Hence, expected trading needs $\left\{\left|E\left[\bar{q}_{0, k}\right]-E\left[q_{0, k}^{i}\right]\right|\right\}_{i, k}$ matter for efficient market structure.

${ }^{48}$ Corollary 4 illustrates how the market structure reduces the trading costs (i.e., the joint condition described below Proposition 3 ) with $K=2$. The eigenvector associated with a negative eigenvalue of the surplus matrix difference $\mathbf{Y}\left(\boldsymbol{\Lambda}^{c}\right)-\mathbf{Y}(\widehat{\boldsymbol{\Lambda}})$ is $(1,1)$ if $\rho>0$ and $(1,-1)$ if $\rho<0$. Condition (i) in Corollary 4 shows that the signs of the eigenvector's elements (either $(1,1)$ or $(1,-1)$ ) match the signs of relative trading needs, and the length of interval $[\underline{\xi}, \bar{\xi}]$ in condition (ii) represents how close the trading needs must be to the eigenvector, i.e., how much heterogeneity in trading needs can be accommodated for independent market clearing to yield
} 


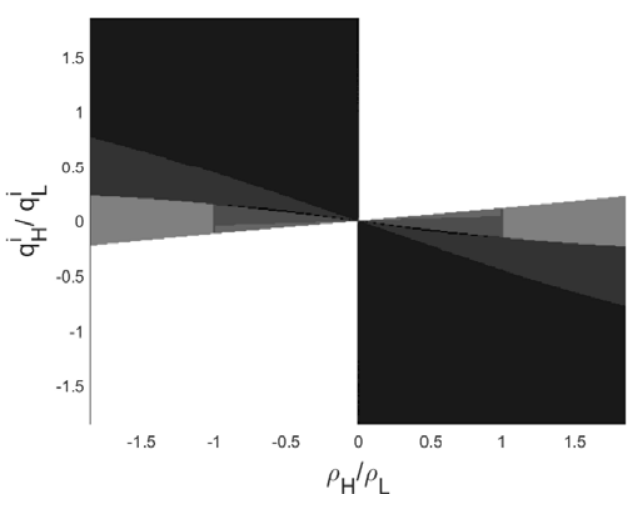

(A) $\rho_{L}=0.2$

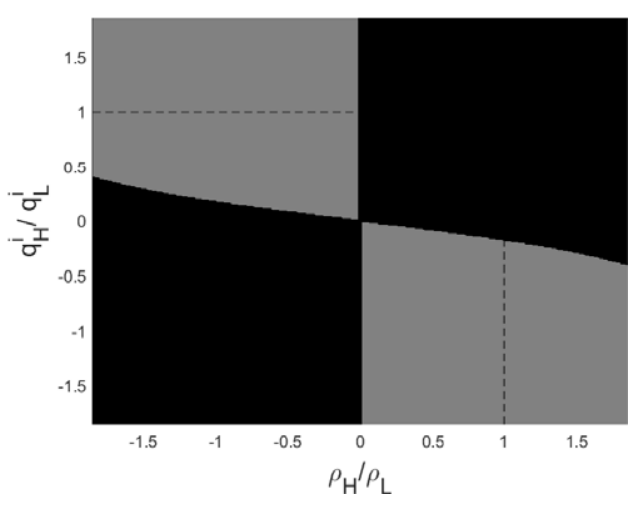

(B) $\rho_{L}=-0.2$

FIGURE 2.-Heterogeneous asset correlations and trading needs. Notes: Each color indicates which market structure provides the highest ex ante welfare. Darker colors represent market structures with more demand conditioning. Panel (A): White $=\{\{1\},\{2\},\{3\}\}$ (i.e., the uncontingent market); Shades of gray $=\{\{1,2\},\{3\}\}$, $\{\{1,2\},\{2\},\{3\}\},\{\{1,2\},\{1\},\{3\}\},\{\{1,2\},\{1,3\}\},\{\{1,2\},\{1,3\},\{3\}\}$; and Black $=\{\{1,2\},\{1,3\},\{1\}\}$. Panel (B): Gray $=\{\{1\},\{2,3\}\}$ and Black $=\{\{1,2,3\}\}$ (i.e., the contingent market). Appendix B presents a color version of Figure 2. The welfare effect of the inference error is sufficiently small not to dominate the welfare benefit from diversification $\left(\sigma_{c v}=0, \sigma_{p v}=0.01\right)$. The number of traders is $I=10$. The trading needs for assets 2 and 3 are $\left|E\left[\bar{q}_{0, L}\right]-E\left[q_{0, L}^{i}\right]\right|=1$ for all $i$. Panel (A) assumes the asset payoff correlation $\rho_{L}=0.2$ (i.e., substitutes), and panel (B) assumes $\rho_{L}=-0.2$ (i.e., complements).

COROLLARY 4-Welfare With Multiple Exchanges Versus Joint Market Clearing $(K=2)$ : Consider a market with two imperfectly correlated (i.e., $0<|\rho|<1)$ assets whose variances are the same (i.e., $\sigma_{11}=\sigma_{22}$ ). Suppose there is no inference error, that is, $\left(\sigma_{c v}, \sigma_{p v}\right) \rightarrow \mathbf{0}$ and $\sigma_{0}<1$. The ex ante welfare is strictly larger in the Pareto sense in the uncontingent market $\{\{1\},\{2\}\}$ than in a single exchange for all assets $\{\{1,2\}\}$ if and only if the following conditions hold:

(i) $\operatorname{sign}\left(E\left[\bar{q}_{0,1}\right]-E\left[q_{0,1}^{i}\right]\right) \operatorname{sign}\left(E\left[\bar{q}_{0,2}\right]-E\left[q_{0,2}^{i}\right]\right)=\operatorname{sign}(\rho)$, that is, the market is onesided when $\rho>0$ or two-sided when $\rho<0$; and

(ii) The trading needs are sufficiently symmetric across assets, that is, there exist bounds $\underline{\xi}(\rho, I)<1<\bar{\xi}(\rho, I)$ on the relative trading needs such that

$$
\underline{\xi}(\rho, I)<\left|\frac{E\left[\bar{q}_{0, k}\right]-E\left[q_{0, k}^{i}\right]}{E\left[\bar{q}_{0, \ell}\right]-E\left[q_{0, \ell}^{i}\right]}\right|<\bar{\xi}(\rho, I) \quad \forall i .
$$

Even with a vanishing (cf. ft. 48) endowment variance $\left(\sigma_{c v}, \sigma_{p v}\right)$, the effects of a change in market structure do not vanish, because of the heterogeneity in the mean endowments $\left\{E\left[\mathbf{q}_{0}^{i}\right]\right\}_{i}$. Given the nonzero trading needs $\left\{E\left[\overline{\mathbf{q}}_{0}\right]-E\left[\mathbf{q}_{0}^{i}\right]\right\}_{i}$, by condition (i), the zero cross-asset price impact is beneficial; by condition (ii), the benefit of diversification (i) dominates the cost of risk sharing (cf. Example 1 and Corollary 3 ). ${ }^{49}$

higher welfare. Corollary 4's result is continuous with respect to the variance of endowment distribution and holds away from the limit of zero inference error.

${ }^{49}$ As $I \rightarrow \infty$, the bounds in condition (ii) $\underline{\xi}(\rho, I) \rightarrow 0$ and $\bar{\xi}(\rho, I) \rightarrow \infty$ for all $\rho$, and so the cost of risk sharing goes to zero regardless of the heterogeneity in trading needs across assets. However, $\mathbf{Y}\left(\boldsymbol{\Lambda}^{c}\right)-\mathbf{Y}(\widehat{\boldsymbol{\Lambda}}) \rightarrow$ $\mathbf{0}$, that is, the benefit from diversification relative to the contingent market also vanishes, and the ex ante welfare in $\{\{1\},\{2\}\}$ and $\{\{1,2\}\}$ can differ in the limit only due to the inference error (if $\sigma_{c v}>0$ and $\sigma_{p v}>0$ ). 
EXAMPLE 5-Heterogeneity and Efficient Market Structure: Consider a market with $K=3$ assets, two of which (i.e., 2 and 3 ) are each symmetrically correlated with other assets and have symmetric ex ante trading needs. There are thirteen payoff-relevant market structures, including the contingent and uncontingent ones. Figure 2 plots the welfaremaximizing design as a function of the heterogeneity in asset correlations $\rho_{12} / \rho_{23}=$ $\rho_{13} / \rho_{23} \equiv \rho_{H} / \rho_{L}$ on the horizontal axis and the heterogeneity in trading needs $\left(E\left[\bar{q}_{0,1}\right]-\right.$ $\left.E\left[q_{0,1}^{i}\right]\right) /\left(E\left[\bar{q}_{0,2}\right]-E\left[q_{0,2}^{i}\right]\right)=\left(E\left[\bar{q}_{0,1}\right]-E\left[q_{0,1}^{i}\right]\right) /\left(E\left[\bar{q}_{0,3}\right]-E\left[q_{0,3}^{i}\right]\right) \equiv q_{H}^{i} / q_{L}^{i}$ for all $i$ on the vertical axis.

(a) If the asset correlations and trading needs are symmetric across assets (i.e., the point $\left(\frac{\rho_{H}}{\rho_{L}}, \frac{q_{H}^{i}}{q_{L}^{i}}\right)=(1,1)$ in each panel), either the contingent or the uncontingent market structure is efficient.

(b) In one-sided markets (i.e., when traders either buy or sell all assets), efficient market structure depends on the asset payoff substitutability:

If asset payoffs are complements (i.e., $\rho_{H}<0$ and $\rho_{L}<0$; Figure 2(B)) and traders buy both assets (i.e., $q_{H}^{i}>0$ and $q_{L}^{i}>0$ ), then the contingent market maximizes welfare, irrespective of the heterogeneity in $\left\{\rho_{H}, \rho_{L}\right\}$ and $\left\{q_{H}^{i}, q_{L}^{i}\right\}$.

If asset payoffs are substitutes (i.e., $\rho_{H}>0$ and $\rho_{L}>0$; Figure 2(A)), then the heterogeneity in trading needs matters. If trading needs are symmetric, the uncontingent market is efficient. With sufficiently heterogeneous trading needs, a market structure other than the contingent or uncontingent ones maximizes welfare.

(c) Welfare-maximizing market structures in which some but not all assets clear jointly either link assets to reduce the trading cost of diversification for those assets (orange and blue areas) or link assets with the most heterogeneous trading needs to balance the tradeoff between risk sharing and diversification-even when linking the assets increases the trading costs due to diversification (yellow and purple areas).

Proposition S3 in Appendix C.1 generalizes Corollary 4 to $K \geq 2$ assets. It shows the link between asset payoff substitutes and complements, and the optimality of an extreme market structure in symmetric trading environments. ${ }^{50}$ The uncontingent market is the most efficient when asset payoffs are substitutes $(\rho>0$ in condition (i)), whereas a single exchange for all assets is the most efficient when asset payoffs are complements $(\rho<0)$.

\section{DISCUSSION}

The effects identified in this paper contribute to the discussion concerning the impact of changes in trading technology (e.g., Pagano (1989), Budish, Cramton, and Shim (2015), Pagnotta and Philippon (2018), Budish, Lee, and Shim (2020), Cespa and Vives (forthcoming)). By accommodating general cross-asset demand conditioning, our analysis takes a step towards exploring the implications of innovation in trading technology defined by changes in market clearing that cross-asset conditioning makes possible. We conclude with possible directions for future research and further discussion of the model.

First, the non-neutrality of innovation in trading technology is a manifestation of a more general implication of independence in market clearing: equilibrium payoffs can be changed by innovations whose payoffs lie in the span of traded assets. This paper's model and equilibrium characterization as a fixed point in price impacts can be adapted

\footnotetext{
${ }^{50}$ That is, symmetric demergers (Definition 5), asset covariances (i.e., $\sigma_{k k}=\sigma$ for all $k$ and $\sigma_{k \ell}=\sigma \rho$ for all $\ell \neq k$ and $k$ ), and trading needs across assets $\left(\frac{E\left[\bar{q}_{0, k}\right]-E\left[q_{0, k}^{i}\right]}{E\left[\bar{q}_{0, \ell}\right]-E\left[q_{0, \ell}^{i}\right]}=1\right.$ for all $k, \ell$, and $i$ in condition (ii) of Corollary 4).
} 
to the study of other innovations. In Rostek and Yoon (2020a), we studied one such class: derivatives, that is, securities whose payoffs are defined as bundles (linear combinations) of the existing assets. We showed that the equilibrium effects of the introduction of nonredundant derivatives differ from those produced by the innovation in trading technology studied in this paper. Thus, innovation in trading technology provides an instrument for impacting markets' performance separate from security innovation.

Second, it would be worthwhile to explore whether, in a dynamic market, a joint design of the trading frequency and trading technology could further improve the lower bound on welfare relative to the contingent design. A study of the joint design would also illuminate the effectiveness of traders' breaking up their orders across trading protocols versus over time to mitigate their trading costs. Dynamic trading provides additional reasons to innovate in trading technology (see Lyu, Rostek, and Yoon (2021)). More generally, conditioning on simultaneously determined prices (i.e., this paper) and past prices within or outside an exchange will interact in nontrivial ways with the relative frequencies of market clearing, shocks which renew the gains from trade, and payoff realizations (i.e., consumption). The rich set of market design questions raised by these two types of demand conditioning merits a separate study.

Third, independence in market clearing gives rise to equilibrium price differentials for the same assets, which would be absent with contingent demands (Lemma S4 in Appendix B). This result indicates a possible source of latency arbitrage and competition for speed, which have been controversial (e.g., Budish, Lee, and Shim (2020)). ${ }^{51}$ Our analysis also suggests a potential market-design solution that could eliminate these incentives: demands contingent on simultaneously determined prices eliminate the rents extracted by fast traders. Cross-asset conditioning allows traders to "react" instantaneously to changes in prices within and across exchanges. Since such rents are substantial, it would be worth thinking about the role of exchange design through its effect on price impacts and the presence of such arbitrage opportunities.

Fourth, the non-neutrality of innovation in trading technology could be leveraged to enhance revenue or other objectives in markets such as that for Treasury bills, in which securities are often traded simultaneously and independently.

Fifth, dispensing with the assumption that demands are contingent also admits asymmetric designs: if the demand for asset $k$ is contingent on the price of asset $l$, the converse need not hold. Designs in which demand conditioning is not mutual give rise to additional reasons to innovate, some of which are explored in Lyu, Rostek, and Yoon (2021).

Finally, there is room for further development of the asset-pricing implications of independence in market clearing as well as the equilibrium properties it induces, such as the transformation of risk made possible by the non-proportionality and asymmetry of price impact.

Independence of market clearing: incentives of traders versus exchanges. While the paper does not characterize the endogenous formation of exchanges, the analysis suggests that it is interesting to distinguish between the incentives of traders and exchanges. If traders themselves could decide whether to submit contingent or uncontingent demands,

\footnotetext{
${ }^{51}$ In particular, it is useful to distinguish between the incentives of traders participating in the market to trade fundamental risk versus algorithmic market makers with a speed advantage. We thank an anonymous referee for this suggestion. Since in imperfectly competitive markets, profits from arbitrage are bounded, price equalization (with one shock) requires multiple trading rounds. With additional shocks renewing the gains from trade, price differentials and hence the incentives to profit from them will remain. (See Rostek and Weretka (2015) for the analysis of the incentives of the hedgers vs. outside investors.)
} 
individual optimization entails that a contingent demand would be a best response, taking as given the demand schedules submitted by others. Submission of contingent demand schedules by all traders would be the unique equilibrium, eliminating the welfareimproving (possibly in the Pareto sense) effects of limited conditioning. Thus, implementation of uncontingent trading involves a restriction of the cross-asset conditioning of simultaneously placed orders. This is the prevalent practice. Exchanges, however, endowed with an objective to maximize (e.g., revenue, volume, or liquidity) generally have at least weak incentives not to allow for full demand conditioning of assets traded. Too much innovation, through its externalities on the liquidity of the traded assets, can hinder the exchanges' objective.

Role of the uniform-price mechanism. One might wonder whether the effects of crossasset conditioning are intrinsic to the designs based on the uniform-price demand submission (e.g., markets for Treasury bills, repo, electricity, spectrum, natural resources). The cross-asset conditioning matters in other designs, such as the limit order book. This is the case unless prices are observable without any delay and submission/withdrawal of limit orders is executed immediately. The key to the welfare effects of new exchanges is the inefficiency of equilibrium allocation due to price impact—given incomplete demand conditioning - which new exchanges alter.

Heterogeneous participation. ${ }^{52}$ Decentralizing a market by allowing some traders to participate in exchanges for only a subset of all assets or trade with only a subset of all traders (while submitting contingent schedules) can increase welfare by reallocating risk across traders, provided that traders' risk preferences differ; with symmetric risk preferences, the centralized market maximizes welfare (Malamud and Rostek (2017)). Our results suggest that restrictions on conditioning can provide an effective instrument to increase welfare in the Pareto sense, by improving risk sharing across traders and/or risk diversification across assets in ways not feasible with heterogeneous participation and contingent trading.

\section{APPENDIX A: EQUILIBRIUM CHARACTERIZATION}

Theorem 5 characterizes the equilibrium for general market structures (Definition 4). We allow endowments to be correlated across assets: $\boldsymbol{\Omega}=\left(\operatorname{Cov}\left[q_{0, k}^{i}, q_{0, \ell}^{i}\right]\right)_{k, \ell} \in \mathbb{R}^{K \times K}$ is a positive definite matrix..$^{53}$ In a market structure $N=\{K(n)\}_{n}$, the distribution of asset returns is jointly Normal, $\mathcal{N}\left(\boldsymbol{\delta}^{+}, \mathbf{\Sigma}^{+}\right)$, where $\boldsymbol{\delta}^{+} \in \mathbb{R}^{\sum_{n} K(n)}$ and $\boldsymbol{\Sigma}^{+} \in \mathbb{R}^{\left(\sum_{n} K(n)\right) \times\left(\sum_{n} K(n)\right)}$. We introduce an indicator matrix $\mathbf{W}$ that represents a market structure $N=\{K(n)\}_{n}$.

DEFINITION 6-Indicator Matrix for Market Structure: An indicator matrix $\mathbf{W} \equiv$ $\left(\mathbf{W}_{n}\right)_{n} \in\{0,1\}^{\left(\sum_{n} K(n)\right) \times K}$ represents a market structure $N=\{K(n)\}_{n}$ if, for each exchange $n$, the $(\ell, k)$ th element of $\mathbf{W}_{n} \in\{0,1\}^{K(n) \times K}$ equals 1 if the $\ell$ th asset in exchange $n$ is asset $k$ and zero otherwise.

We can now write $\boldsymbol{\delta}^{+}=\mathbf{W} \boldsymbol{\delta}$ and $\boldsymbol{\Sigma}^{+}=\mathbf{W} \Sigma \mathbf{W}^{\prime}$.

Notation. We define an operator $[\cdot]_{N}: \mathbb{R}^{\left(\sum_{n} K(n)\right) \times\left(\sum_{n} K(n)\right)} \rightarrow \mathbb{R}^{\left(\sum_{n} K(n)\right) \times\left(\sum_{n} K(n)\right)}$ that maps a matrix $\mathbf{M}$ to a block-diagonal matrix $[\mathbf{M}]_{N}$ with $\left([\mathbf{M}]_{N}\right)_{K(n), K\left(n^{\prime}\right)} \equiv \mathbf{0}$ for $n \neq n^{\prime}$ and $\left([\mathbf{M}]_{N}\right)_{K(n), K(n)} \equiv \mathbf{M}_{K(n), K(n)}$ for any $n$.

\footnotetext{
${ }^{52}$ Babus and Kondor (2018), Babus and Parlatore (2021), and Malamud and Rostek (2017) studied markets with limited participation and contingent contracts.

${ }^{53}$ The characterization results-Theorems 2 and 5, Proposition 2, Corollaries 1 and S1, and Lemmas S2 and S3-allow for general market structures. Theorem 5, Proposition 2, Corollary 1, Lemma S2 also allow for correlated endowments across assets, and heterogeneous risk preferences across traders.
} 
THEOREM 5-Equilibrium: Fixed Point in Demand Schedules; General Design: Consider a market with $N=\{K(n)\}_{n}$ exchanges. In equilibrium, the (net) demand schedules, defined by matrix coefficients $\left\{\boldsymbol{a}^{i}, \mathbf{B}^{i}, \mathbf{C}^{i}\right\}_{i}$, and price impacts $\left\{\boldsymbol{\Lambda}^{i}\right\}_{i}$ are characterized by the following conditions: for each trader $i$,

(i) (Demand coefficients, given price impact) Given price impact matrices $\boldsymbol{\Lambda}^{i} \in$ $\mathbb{R}^{\left(\sum_{n} K(n)\right) \times\left(\sum_{n} K(n)\right)}$, the coefficients of (net) demands $\boldsymbol{a}^{i} \in \mathbb{R}^{\sum_{n} K(n)}, \mathbf{B}^{i} \in \mathbb{R}^{\left(\sum_{n} K(n)\right) \times K}$, and $\mathbf{C}^{i} \in \mathbb{R}^{\left(\sum_{n} K(n)\right) \times\left(\sum_{n} K(n)\right)}$ are characterized by

$$
\begin{aligned}
& \boldsymbol{a}^{i}=\mathbf{C}^{i} \boldsymbol{\delta}^{+}+\left(\mathbf{B}^{i}-\left(\alpha^{i} \boldsymbol{\Sigma}^{+}+\boldsymbol{\Lambda}^{i}\right)^{-1} \mathbf{W} \alpha^{i} \mathbf{\Sigma}\right) E\left[\mathbf{q}_{0}^{i}\right] \\
& -\left(\mathbf{C}^{i}-\left(\alpha^{i} \mathbf{\Sigma}^{+}+\boldsymbol{\Lambda}^{i}\right)^{-1}\right) \\
& \times \underbrace{\left(\sum_{j}\left(\alpha^{j} \mathbf{\Sigma}^{+}+\boldsymbol{\Lambda}^{j}\right)^{-1}\right)^{-1} \sum_{j}\left(\alpha^{j} \mathbf{\Sigma}^{+}+\boldsymbol{\Lambda}^{j}\right)^{-1} \mathbf{W} \alpha^{j} \mathbf{\Sigma} E\left[\mathbf{q}_{0}^{j}\right]}_{=\boldsymbol{\delta}^{+}-E[\mathbf{p}]}, \\
& \mathbf{B}^{i}=\left(\alpha^{i} \boldsymbol{\Sigma}^{+}+\boldsymbol{\Lambda}^{i}\right)^{-1} \mathbf{W} \alpha^{i} \mathbf{\Sigma} \\
& -\left(\left(\alpha^{i} \mathbf{\Sigma}^{+}+\Lambda^{i}\right)^{-1}-\mathbf{C}^{i}\right)\left(\sum_{j} \mathbf{C}^{j}\right)^{-1}\left(\mathbf{B}^{i}+\frac{\sigma_{c v}}{\sigma_{c v}+\sigma_{p v}}\left(\sum_{j \neq i} \mathbf{B}^{j}\right)\right), \\
& \text { Adjustment due to cross-asset inference } \\
& \left.[\left(\mathbf{I d}-\left(\alpha^{i} \mathbf{\Sigma}^{+}+\boldsymbol{\Lambda}^{i}\right) \mathbf{C}^{i}\right)\left(\sum_{j} \mathbf{C}^{j}\right)^{-1} \underbrace{\left(\sum_{j \neq i} \mathbf{B}^{j} \boldsymbol{\Omega}\left(\mathbf{B}^{j}+\frac{\sigma_{c v}}{\sigma_{c v}+\sigma_{p v}} \sum_{h \neq i} \mathbf{B}^{h}\right)\right.}_{\text {Inference coefficient } \operatorname{Var}\left[\mathbf{s}^{-i} \mid \mathbf{q}_{0}^{i}\right]})\right]_{N} \\
& =0 \text {, }
\end{aligned}
$$

where $\mathbf{W} \in\{0,1\}^{\left(\sum_{n} K(n)\right) \times K}$ is the indicator matrix in market $N$ (Definition 6).

(ii) (Correct price impact) Price impact $\boldsymbol{\Lambda}^{i}$ equals the transpose of the Jacobian of trader i’s inverse residual supply:

$$
\mathbf{\Lambda}^{i}=\left(\left(\sum_{j \neq i} \mathbf{C}^{j}\right)^{-1}\right)^{\prime}
$$

Note. With one asset per exchange (i.e., $N=\{\{k\}\}_{k}$ ), the statement of Theorem 5 specializes to that of Theorem 1 .

Lemma 2 in Appendix B shows that asset by asset optimization ${ }^{54}$ brings no loss of generality for optimization with respect to a profile of demands $\left\{\left\{q_{k, n}^{i}(\cdot)\right\}_{k \in K(n)}\right\}_{n}{ }^{55}$ Proposi-

\footnotetext{
${ }^{54}$ That is, trader $i$ 's optimization with respect to demand $q_{k, n}^{i}(\cdot)$ for each asset $k \in K(n)$ in each exchange $n$, taking as given his demands for other assets $\left\{q_{\ell, n}^{i}(\cdot)\right\}_{\ell \in K(n), \ell \neq k}$ in exchange $n$ and $\left\{\left\{q_{\ell, n^{\prime}}^{i}(\cdot): \mathbb{R}^{K\left(n^{\prime}\right)} \rightarrow\right.\right.$ $\left.\mathbb{R}\}_{\ell \in K\left(n^{\prime}\right)}\right\}_{n^{\prime} \neq n}$ in all other exchanges $n^{\prime} \neq n$.

${ }^{55} \mathrm{~A}$ unilateral demand change of trader $i$ is understood as a profile of arbitrary twice continuously differentiable functions $\left\{\Delta q_{k}^{i}(\cdot): \mathbb{R}^{K(n)} \rightarrow \mathbb{R}\right\}_{k}$ so that $\mathbf{q}_{K(n)}^{i}(\cdot)+\Delta \mathbf{q}_{K(n)}^{i}(\cdot)$ are downward-sloping with respect to the contingent variables, that is, the Jacobian $\frac{\partial\left(\mathbf{q}_{K(n)}^{i}(\cdot)+\Delta \mathbf{q}_{K(n)}^{i}(\cdot)\right)}{\partial \mathbf{p}_{K(n)}} \in \mathbb{R}^{K(n) \times K(n)}$ is negative semi-definite.
} 
tion 2 shows that pointwise optimization ${ }^{56}$ is necessary and sufficient for optimization with respect to demand schedules $\mathbf{q}_{K(n)}^{i}(\cdot) \equiv\left(q_{k, n}^{i}(\cdot)\right)_{k \in K(n)}: \mathbb{R}^{K(n)} \rightarrow \mathbb{R}^{K(n)}$ in each exchange $n$.

Proof of Theorem 5: (Equilibrium: Fixed Point in Demand Schedules; General Design). Step 1 (Part (i): Optimization, given residual supply $\mathbf{\Lambda}^{i}$ and $F\left(\mathbf{s}^{-i} \mid \mathbf{q}_{0}^{i}\right)$ ). Because $\mathbf{p}_{K(n)}$ maps one-to-one to $\mathbf{s}_{K(n)}^{-i}$ (as we will show in Step 1.3), the price vector $\mathbf{p}_{K(n)}$ has full support. Proposition 2 and Lemma S2 establish that a trader's pointwise optimization for each asset $k$ and each exchange $n$ is necessary and sufficient for optimization in demand functions (i.e., $\left.\left\{\left\{q_{k, n}^{i}(\cdot)\right\}_{k}\right\}_{n}\right)$ : for each $k$ and $n$,

$$
\max _{q_{k, n}^{i} \in \mathbb{R}} E\left[\boldsymbol{\delta} \cdot\left(\mathbf{q}^{i}+\mathbf{q}_{0}^{i}\right)-\frac{\alpha^{i}}{2}\left(\mathbf{q}^{i}+\mathbf{q}_{0}^{i}\right) \cdot \mathbf{\Sigma}\left(\mathbf{q}^{i}+\mathbf{q}_{0}^{i}\right)-\mathbf{p} \cdot \mathbf{q}^{i} \mid \mathbf{p}_{K(n)}, \mathbf{q}_{0}^{i}\right] \quad \forall \mathbf{p}_{K(n)} \in \mathbb{R}^{K(n)},
$$

given his demands for other assets $\left\{q_{\ell, n}^{i}(\cdot)\right\}_{\ell \neq k \in K(n)}$ and $\left\{q_{\ell, n^{\prime}}^{i}(\cdot)\right\}_{\ell \in K\left(n^{\prime}\right), n^{\prime} \neq n}$, and his residual supply functions for all assets: that is, the distribution of the trader's residual supply intercepts $F\left(\mathbf{s}^{-i} \mid \mathbf{q}_{0}^{i}\right)$ and price impact $\boldsymbol{\Lambda}_{K(n)}^{i} \equiv \frac{d \mathbf{p}_{K(n)}}{d \mathbf{q}_{K(n)}^{i}} \in \mathbb{R}^{K(n) \times K(n)}>0$ for all $n \cdot{ }^{57}$ The first-order condition of trader $i$ in each exchange $n$ is

$$
\begin{gathered}
\boldsymbol{\delta}_{K(n)}^{+}-\alpha^{i} \boldsymbol{\Sigma}_{K(n)} \mathbf{q}_{0}^{i}-\alpha^{i} \boldsymbol{\Sigma}_{K(n)}^{+} E\left[\mathbf{q}^{i} \mid \mathbf{p}_{K(n)}, \mathbf{q}_{0}^{i}\right] \\
=\mathbf{p}_{K(n)}+\Lambda_{K(n)}^{i} \mathbf{q}_{K(n)}^{i} \quad \forall \mathbf{p}_{K(n)} \in \mathbb{R}^{K(n)} .
\end{gathered}
$$

In the quadratic-Gaussian setting, $\boldsymbol{\Lambda}_{K(n)}^{i}$ is a constant matrix for all $n$, given the linearity of residual supply.

Because the first-order condition (31) in exchange $n$ depends on expected trades $E\left[\mathbf{q}^{i} \mid \mathbf{p}_{K(n)}, \mathbf{q}_{0}^{i}\right]$ of other assets, to characterize the best-response demands of trader $i$, $\left\{\mathbf{q}_{K(n)}^{i}(\cdot)\right\}_{n}$, we transform the fixed point among the trader's best-response demands into a fixed point among the trader's demand coefficients, given the residual supplies, $\boldsymbol{\Lambda}^{i}$ and $F\left(\mathbf{s}^{-i} \mid \mathbf{q}_{0}^{i}\right)$ (Step 1). We then endogenize the distribution of the residual supply-and thus all demand coefficients, including expected trades $E\left[\mathbf{q}_{K\left(n^{\prime}\right)}^{i} \mid \mathbf{p}_{K(n)}, \mathbf{q}_{0}^{i}\right]$ for all $n^{\prime} \neq n$ and $n-$ as a function of price impacts $\left\{\boldsymbol{\Lambda}^{i}\right\}_{i}$ (Step 2).

Step 1.1 (Parameterization of trader $i$ 's demands in exchanges $n^{\prime} \neq n$ ). Fix trader $i$ 's demands $\left\{\mathbf{q}_{K\left(n^{\prime}\right)}^{i}(\cdot)\right\}_{n^{\prime} \neq n}$ in exchanges $n^{\prime} \neq n$, and parameterize them as linear functions: for each $n^{\prime} \neq n$,

$$
\mathbf{q}_{K\left(n^{\prime}\right)}^{i}\left(\mathbf{p}_{K\left(n^{\prime}\right)}\right)=\boldsymbol{a}_{K\left(n^{\prime}\right)}^{i}-\mathbf{B}_{K\left(n^{\prime}\right)}^{i} \mathbf{q}_{0}^{i}-\mathbf{C}_{K\left(n^{\prime}\right)}^{i} \mathbf{p}_{K\left(n^{\prime}\right)} \quad \forall \mathbf{p}_{K\left(n^{\prime}\right)} \in \mathbb{R}^{K\left(n^{\prime}\right)},
$$

where $\boldsymbol{a}_{K\left(n^{\prime}\right)}^{i} \in \mathbb{R}^{K\left(n^{\prime}\right)}, \mathbf{B}_{K\left(n^{\prime}\right)}^{i} \in \mathbb{R}^{K\left(n^{\prime}\right) \times K}$, and $\mathbf{C}_{K\left(n^{\prime}\right)}^{i} \in \mathbb{R}^{K\left(n^{\prime}\right) \times K\left(n^{\prime}\right)}$.

Step 1.2 (Expected trades, given $F\left(\mathbf{s}^{-i} \mid \mathbf{q}_{0}^{i}\right)$ ). To endogenize the expected trades in a trader's demands in exchange $n$, we characterize the distributions of prices $\mathbf{p}_{K\left(n^{\prime}\right)}$ and the trader's quantity traded $\mathbf{q}_{K\left(n^{\prime}\right)}^{i}$ in other exchanges $n^{\prime} \neq n$, using the parameterized demands (32).

The price vector $\mathbf{p}_{K\left(n^{\prime}\right)}$ is determined as a function of the residual supply intercept $\mathbf{s}_{K\left(n^{\prime}\right)}^{-i}$ by applying market clearing to trader $i$ 's demands (32) and residual supply $\mathbf{S}_{K\left(n^{\prime}\right)}^{-i}(\cdot)=$

\footnotetext{
${ }^{56}$ That is, optimization with respect to $\mathbf{q}_{K(n)}^{i}$ pointwise to each realization of $\mathbf{s}_{K(n)}^{-i} \in \mathbb{R}^{K(n)}$ in each exchange $n$.

${ }^{57}$ Given the downward-sloping demands of traders $j \neq i$ (Step 2.2).
} 


$$
\begin{aligned}
\mathbf{s}_{K\left(n^{\prime}\right)}^{-i}+ & \left(\left(\boldsymbol{\Lambda}_{K\left(n^{\prime}\right)}^{i}\right)^{-1}\right)^{\prime} \mathbf{p}_{K\left(n^{\prime}\right)}: \mathbb{R}^{K\left(n^{\prime}\right)} \rightarrow \mathbb{R}^{K\left(n^{\prime}\right)} \text { : for each } n^{\prime} \neq n, \\
& \boldsymbol{a}_{K\left(n^{\prime}\right)}^{i}-\mathbf{B}_{K\left(n^{\prime}\right)}^{i} \mathbf{q}_{0}^{i}-\mathbf{C}_{K\left(n^{\prime}\right)}^{i} \mathbf{p}_{K\left(n^{\prime}\right)}=\mathbf{s}_{K\left(n^{\prime}\right)}^{-i}+\left(\left(\boldsymbol{\Lambda}_{K\left(n^{\prime}\right)}^{i}\right)^{-1}\right)^{\prime} \mathbf{p}_{K\left(n^{\prime}\right)} \quad \forall \mathbf{s}_{K\left(n^{\prime}\right)}^{-i} \in \mathbb{R}^{K\left(n^{\prime}\right)} .
\end{aligned}
$$

Given the downward-sloping demand in each exchange $n^{\prime} \neq n$ (i.e., we assume $\mathbf{C}_{K\left(n^{\prime}\right)}^{i}>\mathbf{0}$ ), price vector $\mathbf{p}_{K\left(n^{\prime}\right)}$ maps one-to-one to the residual supply intercept vector $\mathbf{s}_{K\left(n^{\prime}\right)}^{-i}$, which we can thus treat as the contingent variable in trader $i$ 's demands in exchange $n^{\prime}$ (in place of $\left.\mathbf{p}_{K\left(n^{\prime}\right)}\right)$ :

$$
\begin{aligned}
\mathbf{q}_{K\left(n^{\prime}\right)}^{i *}\left(\mathbf{s}_{K\left(n^{\prime}\right)}^{i}\right)= & \left(\mathbf{C}_{K\left(n^{\prime}\right)}^{i}\left(\boldsymbol{\Lambda}_{K\left(n^{\prime}\right)}^{i}\right)^{\prime}+\mathbf{I} \mathbf{d}\right)^{-1}\left(\boldsymbol{a}_{K\left(n^{\prime}\right)}^{i}-\mathbf{B}_{K\left(n^{\prime}\right)}^{i} \mathbf{q}_{0}^{i}\right) \\
& +\mathbf{C}_{K\left(n^{\prime}\right)}^{i}\left(\boldsymbol{\Lambda}_{K\left(n^{\prime}\right)}^{i}\right)^{\prime}\left(\mathbf{C}_{K\left(n^{\prime}\right)}^{i}\left(\boldsymbol{\Lambda}_{K\left(n^{\prime}\right)}^{i}\right)^{\prime}+\mathbf{I d}\right)^{-1} \mathbf{s}_{K\left(n^{\prime}\right)}^{-i} .
\end{aligned}
$$

Equation (34) characterizes the distribution of trades $\mathbf{q}_{K\left(n^{\prime}\right)}^{i}$ as a function of $F\left(\mathbf{s}^{-i} \mid \mathbf{q}_{0}^{i}\right)$, and $\boldsymbol{a}_{K\left(n^{\prime}\right)}^{i}, \mathbf{B}_{K\left(n^{\prime}\right)}^{i}$, and $\mathbf{C}_{K\left(n^{\prime}\right)}^{i}$. Denote this distribution by $\left.F\left(\mathbf{q}_{K\left(n^{\prime}\right)}^{i}\right) \mid \mathbf{q}_{0}^{i}\right)$. Moreover,

$$
E\left[\mathbf{q}_{K\left(n^{\prime}\right)}^{i}\left(\mathbf{p}_{K\left(n^{\prime}\right)}\right) \mid \mathbf{p}_{K(n)}, \mathbf{q}_{0}^{i}\right]=E\left[\mathbf{q}_{K\left(n^{\prime}\right)}^{i *}\left(\mathbf{s}_{K\left(n^{\prime}\right)}^{-i}\right) \mid \mathbf{s}_{K(n)}^{-i}, \mathbf{q}_{0}^{i}\right] .
$$

By Eq. (34), the expected trades vector $E\left[\mathbf{q}_{K\left(n^{\prime}\right)}^{i *}\left(\mathbf{s}_{K\left(n^{\prime}\right)}^{-i}\right) \mid \mathbf{s}_{K(n)}^{-i}, \mathbf{q}_{0}^{i}\right]$ is a linear function of expected intercepts $E\left[\mathbf{s}_{K\left(n^{\prime}\right)}^{-i} \mid \mathbf{s}_{K(n)}^{-i}, \mathbf{q}_{0}^{i}\right]$. Applying the Projection Theorem to the distribution of intercepts $F\left(\mathbf{s}^{-i} \mid \mathbf{q}_{0}^{i}\right)$, the vector of expected intercepts is

$$
E\left[\mathbf{s}_{K\left(n^{\prime}\right)}^{-i} \mid \mathbf{s}_{K(n)}^{-i}, \mathbf{q}_{0}^{i}\right]=\mathbf{x}_{n^{\prime}, n}^{-i}+\mathbf{Y}_{n^{\prime}, n}^{-i} \mathbf{s}_{K(n)}^{-i}+\mathbf{Z}_{n^{\prime}, n}^{-i} \mathbf{q}_{0}^{i},
$$

where $\mathbf{x}_{n^{\prime}, n}^{-i} \in \mathbb{R}^{K\left(n^{\prime}\right)}, \mathbf{Y}_{n^{\prime}, n}^{-i} \in \mathbb{R}^{K\left(n^{\prime}\right) \times K(n)}$, and $\mathbf{Z}_{n^{\prime}, n}^{-i} \in \mathbb{R}^{K\left(n^{\prime}\right) \times K}$ are coefficients of the expected residual supply intercepts. We will endogenize these coefficients in Eqs. (44)-(46), having endogenized distribution $F\left(\mathbf{s}^{-i} \mid \mathbf{q}_{0}^{i}\right)$.

Substituting the expected intercepts (36) into Eq. (35) characterizes the expected trades $E\left[\mathbf{q}^{i} \mid \mathbf{s}_{K(n)}^{-i}, \mathbf{q}_{0}^{i}\right]$ as a function of the demand coefficients in other exchanges $\left\{\boldsymbol{a}_{K\left(n^{\prime}\right)}^{i}, \mathbf{B}_{K\left(n^{\prime}\right)}^{i}, \mathbf{C}_{K\left(n^{\prime}\right)}^{i}\right\}_{n^{\prime} \neq n}$ and the inference coefficients $\left\{\mathbf{x}_{n^{\prime}, n}^{-i}, \mathbf{Y}_{n^{\prime}, n}^{-i}, \mathbf{Z}_{n^{\prime}, n}^{-i}\right\}_{n^{\prime} \neq n}$.

Step 1.3 (Best response in exchange $n$ is linear). Substituting the expected trades (35) into the first-order condition (31) gives the best-response demands $\mathbf{q}_{K(n)}^{i}(\cdot)$ in exchange $n$ as a linear function of $\mathbf{p}_{K(n)}, \mathbf{s}_{K(n)}^{-i}$, and $\mathbf{q}_{0}^{i}$ :

$$
\begin{aligned}
\left(\alpha^{i} \mathbf{\Sigma}_{K(n), K(n)}^{+}+\boldsymbol{\Lambda}_{K(n)}^{i}\right) \mathbf{q}_{K(n)}^{i} & \\
= & \boldsymbol{\delta}_{K(n)}^{+}-\mathbf{p}_{K(n)}-\alpha^{i} \mathbf{\Sigma}_{K(n)} \mathbf{q}_{0}^{i} \\
& -\sum_{n^{\prime} \neq n} \alpha^{i} \mathbf{\Sigma}_{K(n), K\left(n^{\prime}\right)}^{+}\left(\mathbf{C}_{K\left(n^{\prime}\right)}^{i}\left(\boldsymbol{\Lambda}_{K\left(n^{\prime}\right)}^{i}\right)^{\prime}+\mathbf{I d}\right)^{-1}\left(\boldsymbol{a}_{K\left(n^{\prime}\right)}^{i}-\mathbf{B}_{K\left(n^{\prime}\right)}^{i} \mathbf{q}_{0}^{i}\right) \\
& -\sum_{n^{\prime} \neq n} \alpha^{i} \mathbf{\Sigma}_{K(n), K\left(n^{\prime}\right)}^{+} \mathbf{C}_{K\left(n^{\prime}\right)}^{i}\left(\boldsymbol{\Lambda}_{K\left(n^{\prime}\right)}^{i}\right)^{\prime}\left(\mathbf{C}_{K\left(n^{\prime}\right)}^{i}\left(\boldsymbol{\Lambda}_{K\left(n^{\prime}\right)}^{i}\right)^{\prime}+\mathbf{I d}\right)^{-1} \\
& \times\left(\mathbf{x}_{n^{\prime}, n}^{-i}+\mathbf{Y}_{n^{\prime}, n}^{-i} \mathbf{s}_{K(n)}^{-i}+\mathbf{Z}_{n^{\prime}, n}^{-i} \mathbf{q}_{0}^{i}\right) .
\end{aligned}
$$

By the linearity of the downward-sloping best response $\mathbf{q}_{K(n)}^{i}(\cdot)$, equilibrium price $\mathbf{p}_{K(n)}$ is a strictly monotone linear function of $\mathbf{s}_{K(n)}^{-i}$ (Eq. (33) for exchange $n$ ), and hence maps oneto-one to $\mathbf{s}_{K(n)}^{-i}$. Thus, substituting $\mathbf{s}_{K(n)}^{-i}$ for $\mathbf{p}_{K(n)}$ in Eq. (37) gives the best response $\mathbf{q}_{K(n)}^{i}(\cdot)$ 
as a function of his private information $\mathbf{q}_{0}^{i}$ and contingent variable $\mathbf{p}_{K(n)}$. This allows us to parameterize $\mathbf{q}_{K(n)}^{i}(\cdot)$ as a linear function of $\mathbf{q}_{0}^{i}$ and $\mathbf{p}_{K(n)}$ :

$$
\mathbf{q}_{K(n)}^{i}\left(\mathbf{p}_{K(n)}\right)=\boldsymbol{a}_{K(n)}^{i}-\mathbf{B}_{K(n)}^{i} \mathbf{q}_{0}^{i}-\mathbf{C}_{K(n)}^{i} \mathbf{p}_{K(n)} \quad \forall \mathbf{p}_{K(n)} \in \mathbb{R}^{K(n)} .
$$

Step 1.4 (Fixed point for each demand coefficient as a single matrix equation). Given the linearity of best-response schedules $\left\{\mathbf{q}_{K(n)}^{i}(\cdot)\right\}_{n}$ in all exchanges (Step 1.3), we will write a profile of demand schedules $\mathbf{q}^{i}(\cdot)=\left(\mathbf{q}_{K(n)}^{i}(\cdot): \mathbb{R}^{K(n)} \rightarrow \mathbb{R}^{K(n)}\right)_{n}$ in matrix form (Eq. (39) below). The matrix form allows us to write the fixed point problem (37) for trader $i$ 's best-response demands in all exchanges as a system of matrix equations, given $\boldsymbol{\Lambda}^{i}$ and $F\left(\mathbf{s}^{-i} \mid \mathbf{q}_{0}^{i}\right)$.

Define demand coefficients for all $N$ exchanges,

$$
\begin{aligned}
\boldsymbol{a}^{i} & \equiv\left(\boldsymbol{a}_{K(n)}^{i}\right)_{n} \in \mathbb{R}^{\sum_{n} K(n)}, \quad \mathbf{B}^{i} \equiv\left(\mathbf{B}_{K(n)}^{i}\right)_{n} \in \mathbb{R}^{\left(\sum_{n} K(n)\right) \times K}, \\
\mathbf{C}^{i} & \equiv \operatorname{diag}\left(\mathbf{C}_{K(n)}^{i}\right)_{n} \in \mathbb{R}^{\left(\sum_{n} K(n)\right) \times\left(\sum_{n} K(n)\right)} .
\end{aligned}
$$

With matrix coefficients $\left\{\boldsymbol{a}^{i}, \mathbf{B}^{i}, \mathbf{C}^{i}\right\}_{i}$, a profile of trader $i$ 's demand schedules $\mathbf{q}^{i}(\cdot)$ can be written as a function of the vector of the contingent variables in all exchanges $\mathbf{p} \in \mathbb{R}^{\sum_{n} K(n)}$ rather than the price vector $\mathbf{p}_{K(n)} \in \mathbb{R}^{K(n)}$ for exchange $n$ :

$$
\mathbf{q}^{i}(\mathbf{p})=\boldsymbol{a}^{i}-\mathbf{B}^{i} \mathbf{q}_{0}^{i}-\mathbf{C}^{i} \mathbf{p} \quad \forall \mathbf{p} \in \mathbb{R}^{\sum_{n} K(n)},
$$

using that the matrix slope $\mathbf{C}^{i}=\operatorname{diag}\left(\mathbf{C}_{K(n)}^{i}\right)_{n}$ is a block-diagonal matrix; each block corresponds to an exchange in $N$. Similarly, we can write the inference coefficients (36) in trader $i$ 's expected intercepts $E\left[\mathbf{s}_{K\left(n^{\prime}\right)}^{-i} \mid \mathbf{s}_{K(n)}^{-i}, \mathbf{q}_{0}^{i}\right]$ in matrix form:

$$
\left(\mathbf{x}_{n^{\prime}, n}^{-i}\right)_{n^{\prime}} \in \mathbb{R}^{\sum_{n} K(n)}, \quad \mathbf{Y}^{-i} \equiv\left(Y_{n^{\prime}, n}^{-i}\right)_{n^{\prime}, n} \in \mathbb{R}^{\left(\sum_{n} K(n)\right) \times\left(\sum_{n} K(n)\right)}, \quad\left(\mathbf{Z}_{n^{\prime}, n}^{-i}\right)_{n^{\prime}} \in \mathbb{R}^{\left(\sum_{n} K(n)\right) \times K},
$$

where $\mathbf{x}_{n, n}^{-i} \equiv \mathbf{0}, \mathbf{Y}_{n, n}^{-i} \equiv \mathbf{I d}$, and $\mathbf{Z}_{n, n}^{-i} \equiv \mathbf{0}$ for all $n$ and $i$.

Using the matrix demand coefficients $\left\{\boldsymbol{a}^{i}, \mathbf{B}^{i}, \mathbf{C}^{i}\right\}$ and the matrix inference coefficients $\left\{\left\{\left(\mathbf{x}_{n^{\prime}, n}^{-i}\right)_{n^{\prime}},\left(\mathbf{Z}_{n^{\prime}, n}^{-i}\right)_{n^{\prime}}\right\}_{n}, \mathbf{Y}^{-i}\right\}$, the fixed point (37) for trader $i$ 's best-response demands across exchanges simplifies to three matrix equations, one for each demand coefficient:

$$
\begin{aligned}
& \left(\alpha^{i} \boldsymbol{\Sigma}^{+}+\boldsymbol{\Lambda}^{i}+\left(\boldsymbol{\Lambda}^{i}\right)^{\prime}\right)\left(\mathbf{C}^{i}\left(\boldsymbol{\Lambda}^{i}\right)^{\prime}+\mathbf{I d}\right)^{-1} \boldsymbol{a}^{i} \\
& \quad=\boldsymbol{\delta}^{+}-\left(\alpha^{i} \mathbf{\Sigma}_{K(n)}^{+} \mathbf{C}^{i}\left(\boldsymbol{\Lambda}^{i}\right)^{\prime}\left(\mathbf{C}^{i}\left(\boldsymbol{\Lambda}^{i}\right)^{\prime}+\mathbf{I} \mathbf{d}\right)^{-1}\left(\mathbf{x}_{n^{\prime}, n}^{-i}\right)_{n^{\prime}}\right)_{n}, \\
& \left(\alpha^{i} \boldsymbol{\Sigma}^{+}+\boldsymbol{\Lambda}^{i}+\left(\boldsymbol{\Lambda}^{i}\right)^{\prime}\right)\left(\mathbf{C}^{i}\left(\boldsymbol{\Lambda}^{i}\right)^{\prime}+\mathbf{I d}\right)^{-1} \mathbf{B}^{i} \\
& \quad=\mathbf{W} \alpha^{i} \boldsymbol{\Sigma}+\left(\alpha^{i} \boldsymbol{\Sigma}_{K(n)}^{+} \mathbf{C}^{i}\left(\boldsymbol{\Lambda}^{i}\right)^{\prime}\left(\mathbf{C}^{i}\left(\boldsymbol{\Lambda}^{i}\right)^{\prime}+\mathbf{I d}\right)^{-1}\left(\mathbf{Z}_{n^{\prime}, n}^{-i}\right)_{n^{\prime}}\right)_{n}, \\
& \boldsymbol{\Lambda}^{i} \mathbf{C}^{i}+\left[\alpha^{i} \boldsymbol{\Sigma}^{+} \mathbf{C}^{i}\left(\boldsymbol{\Lambda}^{i}\right)^{\prime}\left(\mathbf{C}^{i}\left(\boldsymbol{\Lambda}^{i}\right)^{\prime}+\mathbf{I d}\right)^{-1} \mathbf{Y}^{-i}\right]_{N}\left(\boldsymbol{\Lambda}^{i}\right)^{\prime}\left(\mathbf{C}^{i}\left(\boldsymbol{\Lambda}^{i}\right)^{\prime}+\mathbf{I d}\right)=\mathbf{I d} .
\end{aligned}
$$

Step 2 (Correct residual supply $\left\{F\left(\mathbf{s}^{-i} \mid \mathbf{q}_{0}^{i}\right)\right\}_{i}$ and $\left.\left\{\mathbf{\Lambda}^{i}\right\}_{i}\right)$. Applying market clearing to the best-response demands (38) of traders $j \neq i$ gives the residual supply of trader $i$ in ex- 
change $n$ : for each $\mathbf{p}_{K(n)} \in \mathbb{R}^{K(n)}$,

$$
\mathbf{S}_{K(n)}^{-i}\left(\mathbf{p}_{K(n)}\right) \equiv-\sum_{j \neq i} \mathbf{q}_{K(n)}^{j}\left(\mathbf{p}_{K(n)}\right)=\underbrace{-\sum_{j \neq i}\left(\boldsymbol{a}_{K(n)}^{j}-\mathbf{B}_{K(n)}^{j} \mathbf{q}_{0}^{j}\right)}_{=\mathbf{s}_{K(n)}^{-i}}+\sum_{j \neq i} \mathbf{C}_{K(n)}^{j} \mathbf{p}_{K(n)} .
$$

Step 2.1 (Correct distribution of residual supply intercepts $\left.\left\{F\left(\mathbf{s}^{-i} \mid \mathbf{q}_{0}^{i}\right)\right\}_{i}\right)$. We endogenize the distributions of the vector of the residual supply intercepts $\mathbf{s}^{-i}$ of each trader $i$ as a function of price impacts $\left\{\boldsymbol{\Lambda}^{j}\right\}_{j}$. The vector of intercepts $\mathbf{s}_{K(n)}^{-i}$ in Eq. (43) in each exchange $n$ is jointly Normally distributed:

$$
F\left(\mathbf{s}^{-i} \mid \mathbf{q}_{0}^{i}\right)=\mathcal{N}\left(-\sum_{j \neq i}\left(\boldsymbol{a}^{j}-\mathbf{B}^{j} E\left[\mathbf{q}_{0}^{j} \mid \mathbf{q}_{0}^{i}\right]\right), \sum_{j, h \neq i} \mathbf{B}^{j} \operatorname{Cov}\left[\mathbf{q}_{0}^{j}, \mathbf{q}_{0}^{h} \mid \mathbf{q}_{0}^{i}\right]\left(\mathbf{B}^{h}\right)^{\prime}\right),
$$

given traders' $j \neq i$ demand coefficients $\left\{\boldsymbol{a}^{j}, \mathbf{B}^{j}\right\}_{j \neq i}$ and the primitive joint distribution of their endowments $F\left(\left(\mathbf{q}_{0}^{j}\right)_{j \neq i} \mid \mathbf{q}_{0}^{i}\right)$. Applying the Projection Theorem to the joint distribution $F\left(\mathbf{s}^{-i} \mid \mathbf{q}_{0}^{i}\right)$ determines the inference coefficients $\left(\mathbf{x}_{n^{\prime}, n}^{-i}\right)_{n^{\prime}}, \mathbf{Y}^{-i}$, and $\left(\mathbf{Z}_{n^{\prime}, n}^{-i}\right)_{n^{\prime}}$ in expected intercepts $E\left[\mathbf{s}^{-i} \mid \mathbf{s}_{K(n)}^{-i}, \mathbf{q}_{0}^{i}\right]$ (Eq. (36)) as functions of demand coefficients $\left\{\boldsymbol{a}^{j}, \mathbf{B}^{j}\right\}_{j \neq i}$, given $\left\{\boldsymbol{\Lambda}^{j}\right\}_{j \neq i}$ : for each $n$,

$$
\begin{aligned}
\left(\mathbf{x}_{n^{\prime}, n}^{-i}\right)_{n^{\prime}}= & -\sum_{j \neq i}\left(\boldsymbol{a}^{j}-\left(\mathbf{Y}_{n^{\prime}, n}^{-i}\right)_{n^{\prime}} \boldsymbol{a}_{K(n)}^{j}\right) \\
& +\sum_{j \neq i}\left(\mathbf{B}^{j}-\left(\mathbf{Y}_{n^{\prime}, n}^{-i}\right)_{n^{\prime}} \mathbf{B}_{K(n)}^{j}\right)\left(E\left[\mathbf{q}_{0}^{j}\right]-\frac{\sigma_{c v}}{\sigma_{c v}+\sigma_{p v}} E\left[\mathbf{q}_{0}^{i}\right]\right), \\
\left(\mathbf{Z}_{n^{\prime}, n}^{-i}\right)_{n^{\prime}}= & \frac{\sigma_{c v}}{\sigma_{c v}+\sigma_{p v}} \sum_{j \neq i}\left(\mathbf{B}^{j}-\left(\mathbf{Y}_{n^{\prime}, n}^{-i}\right)_{n^{\prime}} \mathbf{B}_{K(n)}^{j}\right) \\
\mathbf{Y}^{-i}= & \left(\sum_{j \neq i} \mathbf{B}^{j} \boldsymbol{\Omega}\left(\mathbf{B}^{j}+\frac{\sigma_{c v}}{\sigma_{c v}+\sigma_{p v}} \sum_{h \neq i} \mathbf{B}^{h}\right)\right) \\
& \times\left[\sum_{j \neq i} \mathbf{B}^{j} \mathbf{\Omega}^{+}\left(\mathbf{B}^{j}+\frac{\sigma_{c v}}{\sigma_{c v}+\sigma_{p v}} \sum_{h \neq i} \mathbf{B}^{h}\right)^{\prime}\right]_{N}^{-1}
\end{aligned}
$$

Substituting these inference coefficients into Eqs. (40)-(42) gives the system of equations (27)-(29) for demand coefficients $\left\{\boldsymbol{a}^{i}, \mathbf{B}^{i}, \mathbf{C}^{i}\right\}_{i}$ as functions of price impact matrices $\left\{\boldsymbol{\Lambda}^{i}\right\}_{i}$.

Step 2.2 (Part (ii): Correct price impact $\left\{\boldsymbol{\Lambda}^{i}\right\}_{i}$ ). The transpose of the Jacobian of the trader's inverse residual supply $\left(\mathbf{S}^{-i}(\cdot)\right)^{-1}$ characterizes equilibrium price impact $\boldsymbol{\Lambda}^{i} \equiv$ $\operatorname{diag}\left(\boldsymbol{\Lambda}_{K(n)}^{i}\right)_{n}$ by a single matrix equation for all exchanges: for each $i$,

$$
\mathbf{\Lambda}^{i}=\left(\left(\sum_{j \neq i} \mathbf{C}^{j}\right)^{-1}\right)^{\prime} .
$$

The system of equations (28)-(29) and (47) for all traders characterizes the fixed point problem for price impact $\left\{\boldsymbol{\Lambda}^{i}\right\}_{i}$.

Q.E.D. 
Proposition 4 characterizes the traders' equilibrium payoffs. Its proof is provided in Appendix B. Recall that $\operatorname{tr}(\mathbf{M}) \equiv \sum_{k} m_{k k}$ is the trace of a matrix $\mathbf{M}$ (i.e., the sum of its diagonal elements).

Proposition 4-Sufficient Statistic for Equilibrium Payoffs: Let $I<\infty$ and $K>1$. Assume that $\mathbf{\Sigma}$ is not singular. ${ }^{58}$ Fix a market structure $N=\{K(n)\}_{n}$.

(1) (Expected payoffs) The expected equilibrium payoff of trader $i$ is characterized as a function of $\widehat{\boldsymbol{\Lambda}}$ and $\widehat{\mathbf{B}}$ :

$$
\begin{aligned}
E\left[u^{i}\left(\mathbf{q}^{i}\right)-\mathbf{p} \cdot \mathbf{q}^{i}\right]= & \underbrace{E\left[\boldsymbol{\delta} \cdot \mathbf{q}_{0}^{i}-\frac{1}{2} \mathbf{q}_{0}^{i} \cdot \alpha \boldsymbol{\Sigma} \mathbf{q}_{0}^{i}\right]}_{\text {Payoff without trade }}+\underbrace{\left(E\left[\overline{\mathbf{q}}_{0}\right]-E\left[\mathbf{q}_{0}^{i}\right]\right) \cdot \mathbf{Y}(\widehat{\boldsymbol{\Lambda}})\left(E\left[\overline{\mathbf{q}}_{0}\right]-E\left[\mathbf{q}_{0}^{i}\right]\right)}_{\text {Equilibrium supplus from trade }} \\
& +\underbrace{\frac{1 I-1}{2} \sigma_{p v} \operatorname{tr}\left(\widehat{\mathbf{B}}^{\prime} \alpha \boldsymbol{\Sigma}+\alpha \boldsymbol{\mathbf { B }}-\widehat{\mathbf{B}^{\prime}} \alpha \mathbf{\Sigma} \widehat{\mathbf{B}}\right)}_{\text {Payoff term due to } \operatorname{Var}\left[\overline{\mathbf{q}}_{0} \mid \mathbf{q}_{0}^{i}\right]>0},
\end{aligned}
$$

where the per-unit price impact $\widehat{\boldsymbol{\Lambda}} \in \mathbb{R}^{K \times K}$ and cross-asset inference $\widehat{\mathbf{B}} \in \mathbb{R}^{K \times K}$ are defined by conditions (23) and (24), respectively, and characterized by

$$
\begin{aligned}
& \widehat{\boldsymbol{\Lambda}} \equiv(\mathbf{W}^{\prime} \underbrace{\boldsymbol{\Lambda}^{-1}}_{=(I-1) \mathbf{C}^{\prime}} \mathbf{W})^{-1}, \\
& \widehat{\mathbf{B}} \equiv \mathbf{W}^{\prime}(\left(1-\sigma_{0}\right)(\alpha \mathbf{\Sigma}^{+}+\underbrace{\boldsymbol{\Lambda}}_{=\frac{1}{I-1}\left(\mathbf{C}^{-1}\right)^{\prime}})+\sigma_{0}(I-1) \underbrace{\boldsymbol{\Lambda}^{\prime}}_{=\frac{1}{I-1} \mathbf{C}^{-1}})^{-1} \mathbf{W} \alpha \mathbf{\Sigma} ;
\end{aligned}
$$

$\mathbf{Y}(\widehat{\boldsymbol{\Lambda}}) \equiv \frac{1}{2} \alpha \mathbf{\Sigma}\left(\alpha \mathbf{\Sigma}+\widehat{\boldsymbol{\Lambda}}^{\prime}\right)^{-1}\left(\alpha \mathbf{\Sigma}+\widehat{\boldsymbol{\Lambda}}+\widehat{\boldsymbol{\Lambda}}^{\prime}\right)(\alpha \mathbf{\Sigma}+\widehat{\boldsymbol{\Lambda}})^{-1} \alpha \mathbf{\Sigma} \in \mathbb{R}^{K \times K}$ represents the marginal payoff per unit of ex ante trading needs $E\left[\overline{\mathbf{q}}_{0}\right]-E\left[\mathbf{q}_{0}^{i}\right]$.

(2) (Sufficient statistic and symmetry) The sufficient statistic $(\widehat{\boldsymbol{\Lambda}}, \widehat{\mathbf{B}})$ for the equilibrium payoffs (48) reduces to $\widehat{\boldsymbol{\Lambda}}$, equivalently $\widehat{\mathbf{B}}$, if and only if the equilibrium price impact is a symmetric matrix, that is., $\Lambda=\mathbf{\Lambda}^{\prime}$.

\section{REFERENCES}

Afonso, G., AND R. LAGos (2015): “Trade Dynamics in the Market for Federal Funds," Econometrica, 83, 263-313. [2892]

ANTILL, S., AND D. DUfFIE (forthcoming): "Augmenting Markets With Mechanisms," Review of Economic Studies, 88 (4), 1665-1719. [2891]

AtKeson, A. G., A. L. Eisfeldt, AND P.-O. Weill (2015): "Entry and Exit in OTC Derivatives Markets," Econometrica, 83, 2231-2292. [2892]

BABUS, A., AND P. KONDOR (2018): "Trading and Information Diffusion in Over-the-Counter Markets," Econometrica, 86, 1727-1769. [2892,2920]

Babus, A., AND C. PARLATORE (2021): "Strategic Fragmented Markets," Working Paper. [2892,2920]

Bergemann, D., T. HeumanN, AND S. Morris (2021): "Information and Market Power," RAND Journal of Economics, 52, 125-150. [2891]

\footnotetext{
${ }^{58}$ The proof allows for a singular covariance $\boldsymbol{\Sigma}$. Then, the uniqueness of $\widehat{\boldsymbol{\Lambda}}$ and $\widehat{\mathbf{B}}$ holds up to payoff equivalence.
} 
Budish, E., P. CRAMTON, AND J. Shim (2015): "The High-Frequency Trading Arms Race: Frequent Batch Auctions as a Market Design Response," Quarterly Journal of Economics, 130 (4), 1547-1621. [2918]

Budish, E., R. LEE, AND J. SHIM (2020): "A Theory of Stock Exchange Competition and Innovation: Will the Market Fix the Market?" Working Paper. [2888,2918,2919]

CESPA, G. (2004): “A Comparison of Stock Market Mechanisms,” RAND Journal of Economics, 35 (4), $803-823$. [2891]

CESPA, G., AND X. VIVES (forthcoming): "Exchange Competition, Entry, and Welfare," Review of Financial Studies. [2918]

CHANG, B., AND S. ZHANG (2019): “Endogenous Market Making and Network Formation,” Working Paper. [2892]

CHEN, D., AND D. DUfFIE (2021): “Market Fragmentation,” Working Paper. [2891]

Choi, M., A. GAlEOTTI, AND S. Goyal (2017): "Trading in Networks: Theory and Experiment," Journal of the European Economic Association, 15 (4), 784-817. [2892]

Collard-Wexler, A., G. Gowrisankaran, AND R. S. LeE (2019): ““Nash-in-Nash” Bargaining: A Microfoundation for Applied Work," Journal of Political Economy, 127 (1), 163-195. [2892]

Condorelli, D., A. Galeotti, And L. Renou (2017): "Bilateral Trading in Networks," Review of Economic Studies, 84 (1), 82-105. [2892]

Du, S., AND H. ZHU (2017a): "Bilateral Trading in Divisible Double Auctions," Journal of Economic Theory, 167, 285-311. [2891]

- (2017b): "What Is the Optimal Trading Frequency in Financial Markets?," Review of Economic Studies, 84 (4), 1606-1651. [2891]

DufFIE, D. (2018): “Notes on LIBOR Conversion,” Working Paper. [2891]

DufFie, D., N. GARleANU, AND L. H. PEDERSEN (2005): “Over-the-Counter Markets,”Econometrica, 73 (6), 1815-1847. [2892]

Elliott, M. (2015): "Inefficiencies in Networked Markets," American Economic Journal: Microeconomics, 7 (4), 43-82. [2892]

GALE, D. (1986a): “Bargaining and Competition Part I: Characterization,” Econometrica, 54 (4), $785-806$. [2892]

_ (1986b): "Bargaining and Competition Part II: Existence," Econometrica, 54 (4), 807-818. [2892]

Garleanu, N., AND L. Pedersen (2013): "Dynamic Trading With Predictable Returns and Transaction Costs," Journal of Finance, 68 (6), 2309-2340. [2891]

Glode, V., AND C. OpP (2016): "Asymmetric Information and Intermediation Chains," American Economic Review, 106 (9), 2699-2721. [2892]

Gofman, M. (2018): “A Network-Based Analysis of Over-the-Counter Markets,” Working Paper. [2892]

HART, O., AND J. TIROLE (1990): "Vertical Integration and Market Foreclosure,” Brookings Papers on Economic Activity, Special Issue, 205-276. [2892]

Horn, H., AND A. Wolinsky (1988): "Bilateral Monopolies and Incentives for Merger," The RAND Journal of Economics, 19 (3), 408-419. [2891]

HugOnNIER, J., B. LESTER, AND P.-O. WeILl (2020): "Frictional Intermediation in Over-the-Counter Markets," Review of Economic Studies, 87, 1432-1469. [2892]

KAWAKAMI, K. (2017): "Welfare Consequences of Information Aggregation and Optimal Market Size," American Economic Journal: Microeconomics, 9 (4), 303-323. [2892]

KLEMPERER, P., AND M. MEYER (1989): "Supply Function Equilibria in Oligopoly Under Uncertainty," Econometrica, 57 (6), 1243-1277. [2888,2895,2896]

Kranton, R. E., AND D. F. Minehart (2001): "A Theory of Buyer-Seller Networks," American Economic Review, 91 (3), 485-508. [2892]

KYLE, A. S. (1989): “Informed Speculation and Imperfect Competition,” Review of Economic Studies, 56 (3), 517-556. [2888,2891,2895,2896]

KYLE, A. S., AND J. LEE (2018): "Information and Competition With Speculation and Hedging,” Working Paper. [2891]

Kyle, A. S., A. A. Obizhaeva, And Y. Wang (2017): "Smooth Trading With Overconfidence and Market Power," Review of Economic Studies, 85 (1), 611-662. [2891]

LAMBERT, N., M. OSTROVSKY, AND M. PANOV (2018): "Strategic Trading in Informationally Complex Environments," Econometrica, 86 (4), 1119-1157. [2904]

Lester, B., A. Shourideh, V. Venkateswaran, And A. Zetlin-Jones (2018): “Market-Making With Search and Information Frictions," Working Paper. [2893]

(2019): "Screening and Adverse Selection in a Frictional Markets," Journal of Political Economy, 127 (1), 338-377. [2893] 
LYU, C., M. RosteK, AND J. H. YoOn (2021): "Innovation in Market Clearing Technology: Design of MultiAsset Trading Mechanisms,” Working Paper. [2891,2919]

Malamud, S., AND M. RosteK (2017): "Decentralized Exchange," American Economic Review, 107 (11), 3320-3362. [2892,2895,2904,2920]

PaGANO, M. (1989): “Trading Volume and Asset Liquidity," Quarterly Journal of Economics, 104 (2), 255-274. [2918]

PAGNOTTA, E., AND T. PHiLIPPON (2018): “Competing on Speed,” Econometrica, 86 (3), 1067-1115. [2918]

PeIVANDI, A., AND R. Vohra (2021): "Instability of Centralized Markets," Econometrica, 89 (1), 163-179. [2892]

RosteK, M., AND M. WeretKa (2015): “Dynamic Thin Markets," Review of Financial Studies, 28 (10), 29462992. [2891,2895,2919]

RosteK, M., J. H Yoon (2021): “Supplement to “Exchange Design and Efficiency',” Econometrica Supplemental Material, 89, https://doi.org/10.3982/ECTA16537. [2889]

RoSTEK, M., AND J. H. YOON (2019): "Dynamic Imperfectly Competitive Markets With Private Information," Working Paper. [2891]

_ (2020a): "Design of Synthetic Financial Products in Decentralized Markets," Working Paper. [2919]

2891] (2020b): "Equilibrium Theory of Financial Markets: Recent Developments," Working Paper. [2889,

SANNIKOV, Y., AND A. SKRZYPACZ (2016): “Dynamic Trading: Price Inertia and Front-Running,” Working Paper. [2891]

VAYANOS, D. (1999): "Strategic Trading and Welfare in a Dynamic Market," Review of Economic Studies, 66 (2), 219-254. [2891]

VAYANOS, D., AND P.-O. WeILl (2008): “A Search-Based Theory of the on-the-Run Phenomenon,” Journal of Finance, 63 (3), 1351-1389. [2892]

VIVES, X. (2011): "Strategic Supply Function Competition With Private Information,” Econometrica, 79 (6), 1919-1966. [2888,2891]

WILSON, R. (1979): “Auctions of Shares,” Quarterly Journal of Economics, 93 (4), 675-689. [2888]

WiTTwer, M. (2021): "Connecting Disconnected Financial Markets?” American Economic Journal: Microeconomics, 13 (1), 252-282. [2891]

ZHANG, A. (2021): "Competition and Manipulation in Derivative Contract Markets," Working Paper. [2891]

ZHu, H. (2014): “Do Dark Pools Harm Price Discovery?” Review of Financial Studies, 27 (3), 747-789. [2892]

(2018a): “A Multi-Maturity Clock Auction Design, Market Design Proposal.” [2891]

(2018b): "Design of CCP Default Management Auctions, Market Design Proposal.” [2891]

\section{Co-editor Alessandro Lizzeri handled this manuscript.}

Manuscript received 24 July, 2018; final version accepted 18 July, 2021; available online 26 July, 2021. 